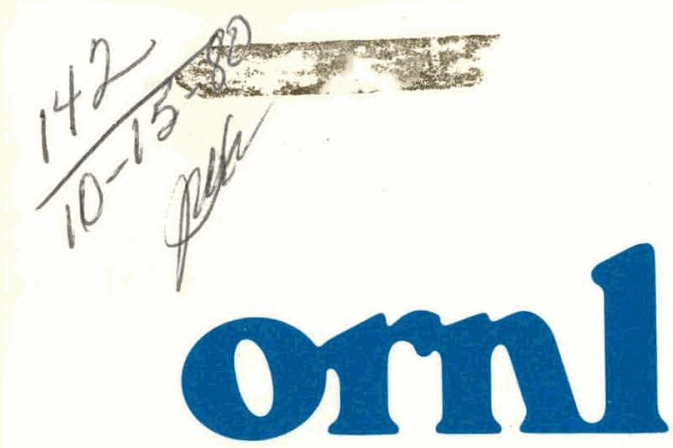

OAK RIDGE NATIONAL LABORATORY

UNION CARBIDE

\section{The Instrumentation and Controls of an Ignited Tokamak}

OPERATED BY

UNION CARBIDE CORPOBATION FOR THE UNITED STATES DEPARTMENT OF ENERGY
W. R. Becraft
J. Golzy
W. A. Houlberg
C. A. Kukielka
R. J. Onega
G. V. S. Raju
R. S. Stone

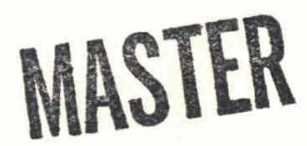




\section{DISCLAIMER}

This report was prepared as an account of work sponsored by an agency of the United States Government. Neither the United States Government nor any agency Thereof, nor any of their employees, makes any warranty, express or implied, or assumes any legal liability or responsibility for the accuracy, completeness, or usefulness of any information, apparatus, product, or process disclosed, or represents that its use would not infringe privately owned rights. Reference herein to any specific commercial product, process, or service by trade name, trademark, manufacturer, or otherwise does not necessarily constitute or imply its endorsement, recommendation, or favoring by the United States Government or any agency thereof. The views and opinions of authors expressed herein do not necessarily state or reflect those of the United States Government or any agency thereof. 


\section{DISCLAIMER}

Portions of this document may be illegible in electronic image products. Images are produced from the best available original document. 


\section{Printed in the United States of America. Available from National Technical Information Service \\ II S Department of Commerce 5285 Port Royal Road, Springfield, Virginia 22161 NTIS price codes-Printed Copy: A07 Microfiche A01}

This report was prepared as an account of work sponsored by an agency of the United States Government. Neither the United States Government nor any agency thereof, nor any of their employees, makes any warranty, express or implied, or assumes any legal liability or responsibility for the accuracy, completeness, or usefulness of any information, apparatus, product, or process disclosed, or represents that its use would not infringe privately owned rights. Reference herein to any specific commercial product, process, or service by trade name, trademark, manufacturer, or otherwise, does not necessarily constitute or imply its endorsement, recommendation, or favoring by the United States Government or any agency thereof. The views and opinions of authors expressed herein do not necessarily state or reflect those of the United States Government or any agency thereof. 
ORNL/TM-7153

Dist. Category UC-20d

Contract No. W-7405-eng-26

FUSION ENERGY DIVISION

THE INSTRUMENTATION AND CONTROLS

OF AN IGNITED TOKAMÁK
W. R. Becraft
J. Golzy
W. A. Houlberg
C. A. Kukielka
R. J. Onega
G. V. S. Raju
R. S. Stone

Date Published: October 1980

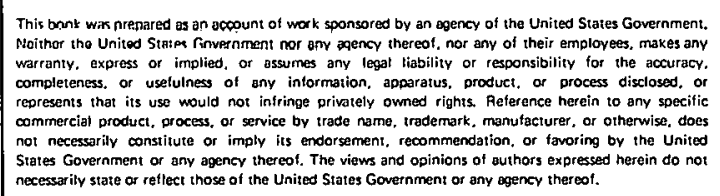

necessarily staze or reflect those of the United States Government or any agency thereot.

Prepared by the OAK RIDGE NATIONAL LABORATORY

Oak Ridge, Tennessee 37830 operated by UNION CARBIDE CORPORATION

for the DEPARTMENT OF ENERGY 


\section{THIS PAGE}

\section{WAS INTENTIONALLY \\ LEFT BLANK}


CONTENTS

ABSTRACT $\ldots \ldots \ldots \ldots \ldots \ldots \ldots \ldots \ldots \ldots \ldots \ldots \ldots \ldots \ldots \ldots \ldots \ldots \ldots \ldots \ldots \ldots \ldots \ldots$

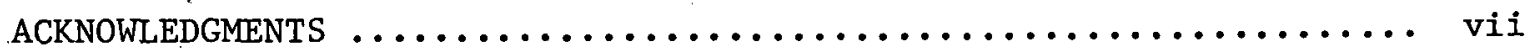

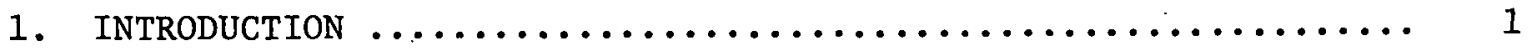

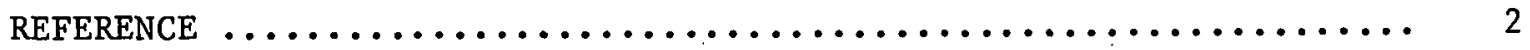

2. PHYSICS ISSUES OF FUELING CONTROL $\ldots \ldots \ldots \ldots \ldots \ldots \ldots \ldots \ldots \ldots \ldots$

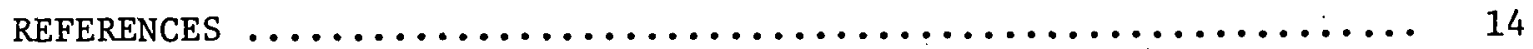

3. COMPUTER SIMULATION OF ISX-B PLASMA CURRENT AND HORIZONTAL

POSITION CONTROL ................................. 15

3.1 SIMULATOR STUDIES OF TOKAMAK CONTROL .............. 15

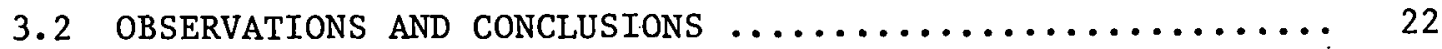

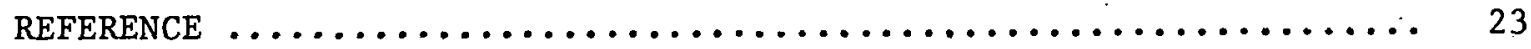

4. MULTIVARIABLE FEEDBACK CONTROL SYSTEM DESIGN FOR ISX-B ...... 25

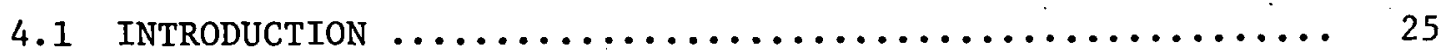

4.2 THE TRANSFER FUNCTION MODEL G(s) FOR ISX-B .......... 27

4.3 THE EXTENDED ROOT LOCUS METHOD ................. 33

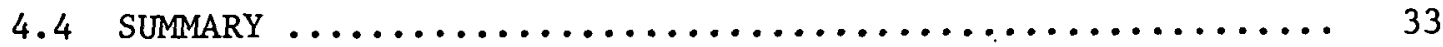

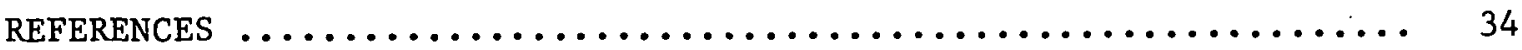

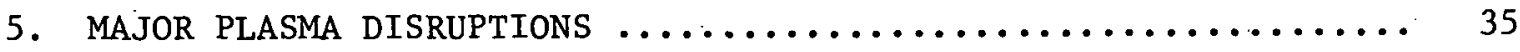

5.1 THE POSSIBILITY OF CONTROL OF AN INCIPIENT MAJOR

PLASMA DISRUPTION ........................... 35

5.2 SUMMARY OF THE CONSEQQUENCES OF A MAJOR PLASMA

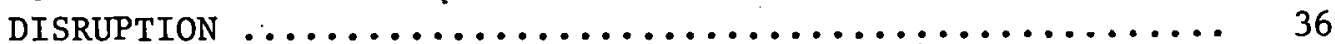

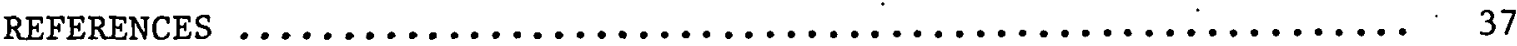

6. DIAGNOSTICS SUITABLE FOR FEEDBACK CONTROL ON ETF $\ldots \ldots \ldots \ldots \ldots .39$

6.1 InTRODUCTION ............................ 39

6.2 TIME CONSTANT INVOLVED IN DIAGNOSTICS AND CONTROLS ..... 40

6.2.1 Major Plasma Disruptions ................. 40

6.2 .2 Power Leve1 Control .................. 41

6.2.3 Position and Shape Control ............... 41

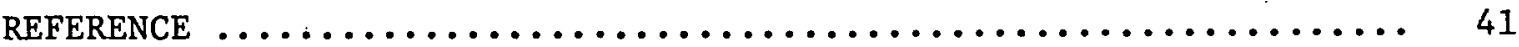

7. ASSESSMENT OF THE CONTROLS R\&D NEEDS $\ldots \ldots \ldots \ldots \ldots \ldots \ldots \ldots \ldots . \ldots 2$

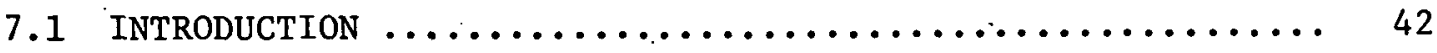

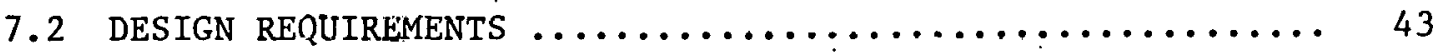

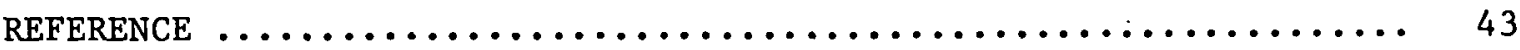




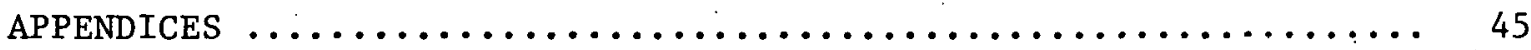

APPENDIX A: CONTROL OF A TOKAMAK FUSION REACTOR:

AN OVERVIEW ............................ 47

A.1 INTRODUCTION ............................... 47

A. 2 STATE VARIABLES AND PERFORMANCE INDICES .............. 52

A. 3 PLASMA STARTUP ..................................... . . . . 54

A.4 PLASMA BURN PHASE ............................. 57

A.5 SHUTDOWN PHASE ............................... 58

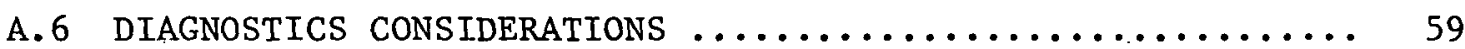

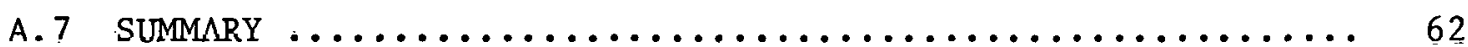

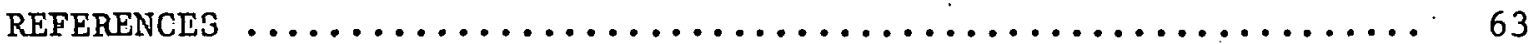

APPENDIX B: THE EXPERIMENTAL DETERMINATION OF THE DEUTERON-

TRITON IN A FUSION PLASMA ..................... 65

REFERENCES ........................................ 75

APPENDIX $\mathrm{C}$ : THE FEASIBILITY OF USING SYNCHROTRON RADIATION AS A FINE-TUNING TEMPERATURE CONTROL . . . . . . . . . . 77

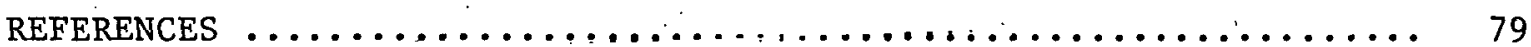

APPENDIX D: THE POSSIBILITY OF EMPLOYING A DIVERTOR

IN A FEEDBACK CONTROL LOOP ................... 83

D. 1 INTRODUCTION ................................... 83

D. 2 DIAGNOSTICS FOR DIVERTOR CONTROL ................... 84

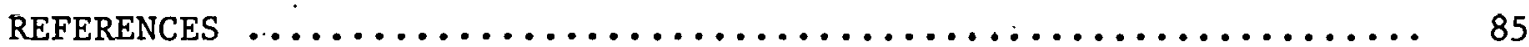

APPENDIX E: THERMAL CONSEQUENCES TO THE FIRST WALL

OF A D-T-FUELED TOKAMAK DUE TO A MAJOR

PLASMA DISRUPTION ........................ 87

E.1 INTRODUC'IION $\ldots \ldots \ldots \ldots \ldots \ldots \ldots \ldots \ldots \ldots \ldots \ldots \ldots \ldots \ldots \ldots \ldots \ldots \ldots$

E. 2 MAJOR PLASMA DISRUPTIONS ......................... 87

E.3 METHODOLOGY ................................... 91

E.4 THE SURFACF. HEAT FLUX APJD VOLUNHE'KLL HEAT GENERATION RATE FOR THE CIRCULAR CHAMBER .............. 94

E.5 RESULTS OF IHE LUMPED PARAMETER MODEL .................. 101

E.6 TEMPERATURE DISTRIBUTION IN THE CIRCULAR

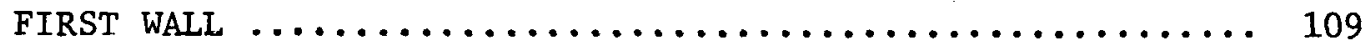

E. 7 THE D-SHAPED VESSEL $\ldots \ldots \ldots \ldots \ldots \ldots \ldots \ldots \ldots \ldots \ldots \ldots \ldots \ldots \ldots \ldots$

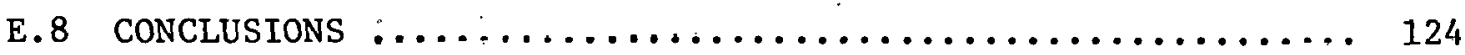

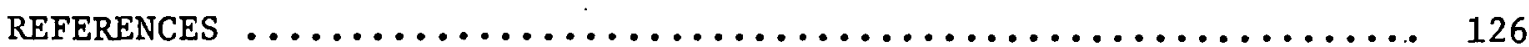


The instrumentation and controls $(I \& C)$ of an ignited plasma magnetically confined in a tokamak configuration needs increased emphasis in the following areas:

(1) physics implications for control,

(2) plasma shaping/position control, and

(3) control to prevent disruptive instabilities.

This document reports on the FY 1979 efforts in these and other areas. Plasma physics studies indicate that feedback control of plasma density can be accomplished primarily by pellet injection rather than by gas puffing. Continued progress has been made in understanding the thermal consequences to the first wall of a major plasma disruption (MPD). Also, a sharpening of the parameters used in the feedback control system of the upgraded Impurity Study Experiment (ISX-B) has been accomplished.

Also presented are discussions in the areas of (1) diagnostics suitable for the Engineering Test Facility (ETF) and (2) future research and development (R\&D) needs. The appendices focus attention on some preliminary ideas about the measurement of the deuteron-triton (D-T) ratio in the plasma, synchrotron radiation, and divertor control. Finally, an appendix documenting the thermal consequences to the first wall of a MPD is presented. 


\section{THIS PAGE}

\section{WAS INTENTIONALLY \\ LEFT BLANK}




\section{ACKNOWLEDGMENTS}

During the investigations that culminated in this report, numerous helpful discussions with fusion colleagues were held. In particular, G. Wiseman, B. Carreras, W. Wells, and J. L. Dunlap gave assistance and guidance to the formulation of some of the ideas in this report. 


\section{INTRODUCTION}

The advancements required for the design and development of an ignited tokamak such as the Engineering Test Facility (ETF), to be operational in the early 1990's, include extensive progress in instrumentation and controls ( $I \& C)$. However, concurrent research and development $(R \& D)$ is required during the machine studies and design evolution, and a control and data-handling philosophy that will permit the required device performance with control simplicity should be established.

Three areas of the I\&C of a tokamak have been examined here in some depth. These efforts are a continuation of previously initiated work. ${ }^{1}$ Some of the significant investigations undertaken are as follows.

(1) Operating scenarios including startup, burn, and shutdown were studied. Density profile control through gas puffing and pellet injection was analyzed using a one-dimensional (1-D) transport code. If the reactor has a divertor, density control will probably have to be accomplished by feedback control of pellet injection rather than by gas puffing .

(2) A hybrid simulation of the upgraded Impurity Study Experiment (ISX-B) horizontal plasma position and current control achieved by changing the internal and external ohmic heating (OH) coil currents has permitted refinements of the tokamak operating parameters. Also, a multivariable feedback control algorithm for ISX-B is being developed for the horizontal plasma position and current control.

(3) The thermal consequences of a major plasma disruption (MPD) to the first wall of a tokamak (the ETF) were analyzed for both circular and D-shaped plasma chambers. Failure in the chamber wall. w1ll, according to these analyses, probably occur after 40-900 disruptions, depending upon the amount the plasma's magnetic center is displaced from the chamber geometric center. A 24-ms disruption time was assumed for the ETF. Each of these areas is discussed more fully in Sects. 2, 3, 4, and 5 .

In addition to these considerations, other aspects of control have been investigated and are presented as appendices. Appendix A contains an overview of the control problem for a deuterium-tritium (D-T)-burning 
tokamak. A design philosophy is required that incorporates the control of the reactor as an integral part of the design tradeoffs.

Appendix $B$ presents some suggestions on methods of measuring deuterontriton ( $D-T)$ ratios. The relative densities of deuterium and tritium in the plasma may not be the same, even though the feed rates are kept the same, because their diffusion rates differ. A measurement of the D-T ratio may be necessary for density control. Appendix B contains some observations on this measurement.

Appendix $\mathrm{C}$ includes a discussion of some preliminary approaches to plasma temperature control. Controlling plasma density by changing the pellet injection rate also changes the plasma temperature. Various ways to regulate the temperature (e.g., neutral beam heating using ripple coils to preterentially remove the alpha particles) have been suggestcd. The feasibility of using a shutter arrangement in the first wall to control synchrotron radiation losses has a1so been examined in a preliminary way.

Appendix D suggests reasons for exploring divertor control to determine its merits and demerits. Finally, Appendix E presents a detailed examination of the thermal consequences to the first wall of a MPD. This information is supplementary to Sect. 5 of this report.

REFEREINCE

1. W. R. Becraft et a1., ORNL/TM-6617, Oak Ridge, Tennessee (1979). 


\section{PHYSICS ISSUES OF FUELING CONTROL}

Fueling scenarios during the burn phase of a tokamak operating cycle have most often assumed that either. frozen hydrogenic pellets or gas puffing can be used as control knobs to maintain the plasma density. However, the technology required to accomplish this control has rarely been addressed. Furthermore, it may be necessary to measure the deuterium and tritium densities separately, process the signals, and adjust the fueling rates of separate deuterium and tritium injection systems in order to maintain a 50-50 plasma. This difficult complication is further discussed in Appendix B.

Here we address some of the differences between pellet and gas injection from the plasma physics viewpoint. There are very great differences between these methods; therefore; different combinations of gas and pellets for deuterium and tritium can provide a wide range of flexibility in the fueling control system. As shown in Table 2.1 there are advantages and disadvantages to each fueling method.

Table 2.1. Advantages and disadvantages of fueling by gas puffing and pellets

\begin{tabular}{|c|c|c|}
\hline & Advantages & Disadvantages \\
\hline Gas puffing. & $\begin{array}{l}\text { Easy to control } \\
\text { Cools edge of plasma } \\
\text { and protects wall }\end{array}$ & $\begin{array}{l}\text { Large fuel turnover so } \\
\text { low fractional burnup } \\
\text { Large amount of tritium } \\
\text { inventory required } \\
\text { Cannot presently meet } \\
\text { demands for teedback } \\
\text { and control }\end{array}$ \\
\hline Pellets & $\begin{array}{l}\text { Fuel deposited more } \\
\text { deeply into the } \\
\text { plasma for efficient } \\
\text { fuel use } \\
\text { Size, velocity, and } \\
\text { repetit.ion rate } \\
\text { variable }\end{array}$ & $\begin{array}{l}\text { Discrete amounts put into } \\
\text { plasma that cause sig- } \\
\text { nificant perturbations } \\
\text { in plasma density and } \\
\text { temperature } \\
\text { Minimum-size pellet re- } \\
\text { quired for penetration } \\
\text { of scrape-off region }\end{array}$ \\
\hline
\end{tabular}


Because a divertor will probably be required on the ETF, fueling in the presence of a bundle divertor with a 13-cm scrape-off thickness was chosen. The machine parameters and other factors used in this study are shown in Table 2.2.

In order to evaluate the effect of the pellets on the plasma, a routine called PELLET ${ }^{l}$ was written to be incorporated as a subroutine in the WHIST code. ${ }^{2}$ A feedback system on the pellet source was used to. keep the average deuterium and tritium densities above the desired level. Pellets were injected into the plasma at $2 \mathrm{~km} / \mathrm{s}$, the effective radius of the pellet being $0.2 \mathrm{~cm}$. Gas puffing of deuterium was used for supplemental fueling but was found to be very inefficient because of the divertor. ${ }^{3}$ The 1-D multifluid transport code WHIST includes ripple enhanced, neoclassical, and anomalous diffusion. SPUDNUT $^{4}$ is a subroutıne that treats the neutrals in the plasma. The neutrals can charge exchange or be ionized. In either case energy is transferred. Particle and energy balances were performed at each of the 37 radial nodes used in the calculation.

It was expected that fueling could be done by pellets, gas puffing, or a combination. However, no stable solution could be obtained when only gas puffing was used. The reason was that the feedback was assumed to be proportional to the difference between the desired density and the actual density. As more gas was puffed into the divertor region, its density built up. As the density increased, ionizations became more prevalent so that more particles were swept to the divertor collector. The consequence was that the gas puffing could not be used as a feedback control on particle density in the bulk of the plasma.

A tradeoff exists between particle pumping ratos and fucling rates by gas puffing and pellet injection. Because of the inadequacy of gas puffing for fueling, it was decided that some deuterium gas puffing would be used to cool the scrape-off region with feedback on the pellet repetition rate to control the plasma density.

Figure 2.1 shows the time-averaged fraction of the deuterium atoms puffed into the divertor region that gets through the scrape-off region and then is ionized in the plasma. The density increases in the scrapeoff region and ionization occurs, causing more particles to be carried 
Table 2.2. Tokamak parameters used in density control via pellet injection and gas puffing

\begin{tabular}{|c|c|}
\hline Parameter & Value \\
\hline Major radius, $R_{0}$ & $4.80 \mathrm{~m}$ \\
\hline Minor radius, a & $1.20 \mathrm{~m}$ \\
\hline Elongation, $\mathrm{b} / \mathrm{a}$ & 1.6 \\
\hline Toroidal ripple, $\delta$ & . \\
\hline Edge & $1.02 \%$ \\
\hline Center & $9.5 \times 10^{-2} \%$ \\
\hline Scrape-off thickness, $d_{i}$ & $13 \mathrm{~cm}$ \\
\hline Pellet radius, $a_{p}$ & $0.2 \mathrm{~cm}$ \\
\hline Pellet speed, $\mathrm{v}_{\mathrm{p}}$ & $2.0 \mathrm{~km} / \mathrm{s}$ \\
\hline Gas puff & $0.03-3.0 \times 10^{23}$ deuterium atoms $/ \mathrm{s}$ \\
\hline $\begin{array}{l}\text { Plasma diffusion } \\
\text { coefficient, } D_{1}\end{array}$ & $\left(1.25 \times 10^{17} / \mathrm{n}_{\mathrm{e}}\right)+5000(\mathrm{r} / \mathrm{a})^{3} \mathrm{~cm}^{2} / \mathrm{s}$ \\
\hline $\begin{array}{l}\text { Divertor diffusion } \\
\text { coefficient, } D_{1}\end{array}$ & $1 / 16(\mathrm{kT} / \mathrm{eB}) \mathrm{cm}^{2} / \mathrm{s}$ \\
\hline
\end{tabular}




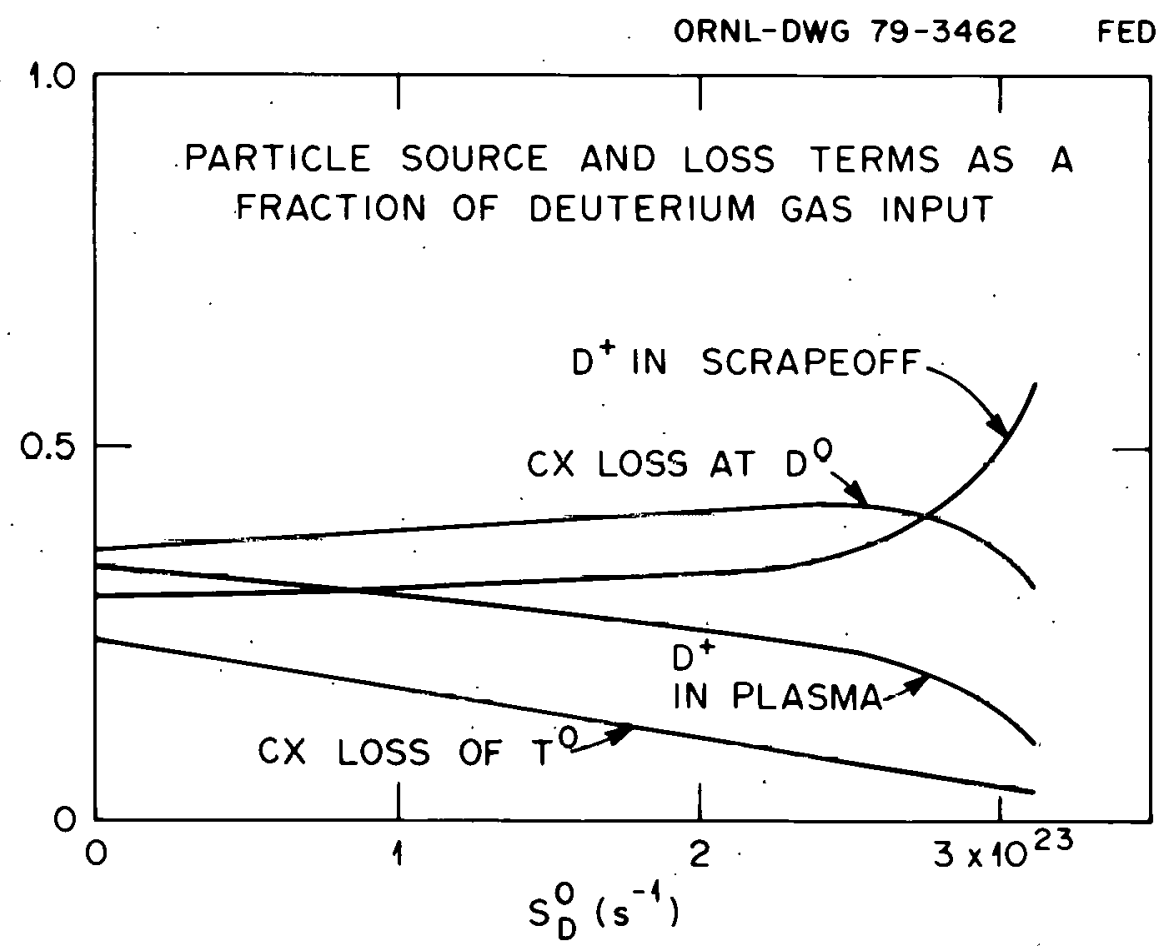

Fig. 2.1. Time-averaged fraction of deuterium gas ionized in the plasma and scrape-off region as the deuterium gas feed rate is increased. At $23 \times 10^{23} \mathrm{~s}^{-1}$ " feed rate of the deuterium, the divertor begins to choke off. 
to the divertor plates as the deuterium gas feed rate is increased. Actually there is a spread in this fraction because pellet injection causes the density to fluctuate. Notice that when the deuterium gas puff rate is $3 \times 10^{23}$ atoms $/ \mathrm{s}$, $\sim 10 \%$ of the deuterium atoms make it to the plasma for ionization. When a pellet is inserted, less of the puffed gas gets into the plasma region.

As the deuterium gas feed rate is increased (see Fig. 2.2), there is initially a drop in the required deuterium pellet fueling; however, this effect diminishes as more neutrals are ionized in the scrape-off region.

Figure 2.3 shows the number of particles from the deuterium gas that is ionized in the scrape-off zone for a $0.5-s$ segment of a typical case. In Figs. 2.3-2.7 the deuterium gas puff rate is held constant at $1.4 \times 10^{23}$ atoms/s. After a pellet is inserted, the amount of neutral gas that is ionized in the scrape-off region increases significantly because the particle density has been increased. Simultaneously, the net particle source in the plasma decreases, as shown in Fig. 2.4. When both the deuterium and the tritium pellets are inserted simultaneously, 280\% of the net deuterium plasma source is in the scrape-off region. The source terms for tritium due to deuterium gas puffing are negative in both the plasma and scrape-off region because of charge exchange losses. The charge exchange neutral flux of deuterium and tritium to the wall is shown in Fig. 2.5.

Figure 2.6 shows the total tritium and deuterium charged particle flow to the divertor. The bulk of the load to the divertor comes shortly after both pellets have been inserted simultaneously. About $3 \times 10^{22}$ deuterons/s strike the divertor collector plate.

The flow of tritium and deuterium to the wall is depicted in Fig. 2.7. of course, to get to the wall the particle must diffuse across field lines whereas it moves parallel to the field lines in the case of flow to the divertor coll.ector plates. When pellets are injected, a large number of particles strike the wall because ionization of the deuterium gas then occurs very close to the wall. This causes sputtering from the wall; however, these considerations have not been built into the present calculations. An extension of these calculations to include particle 


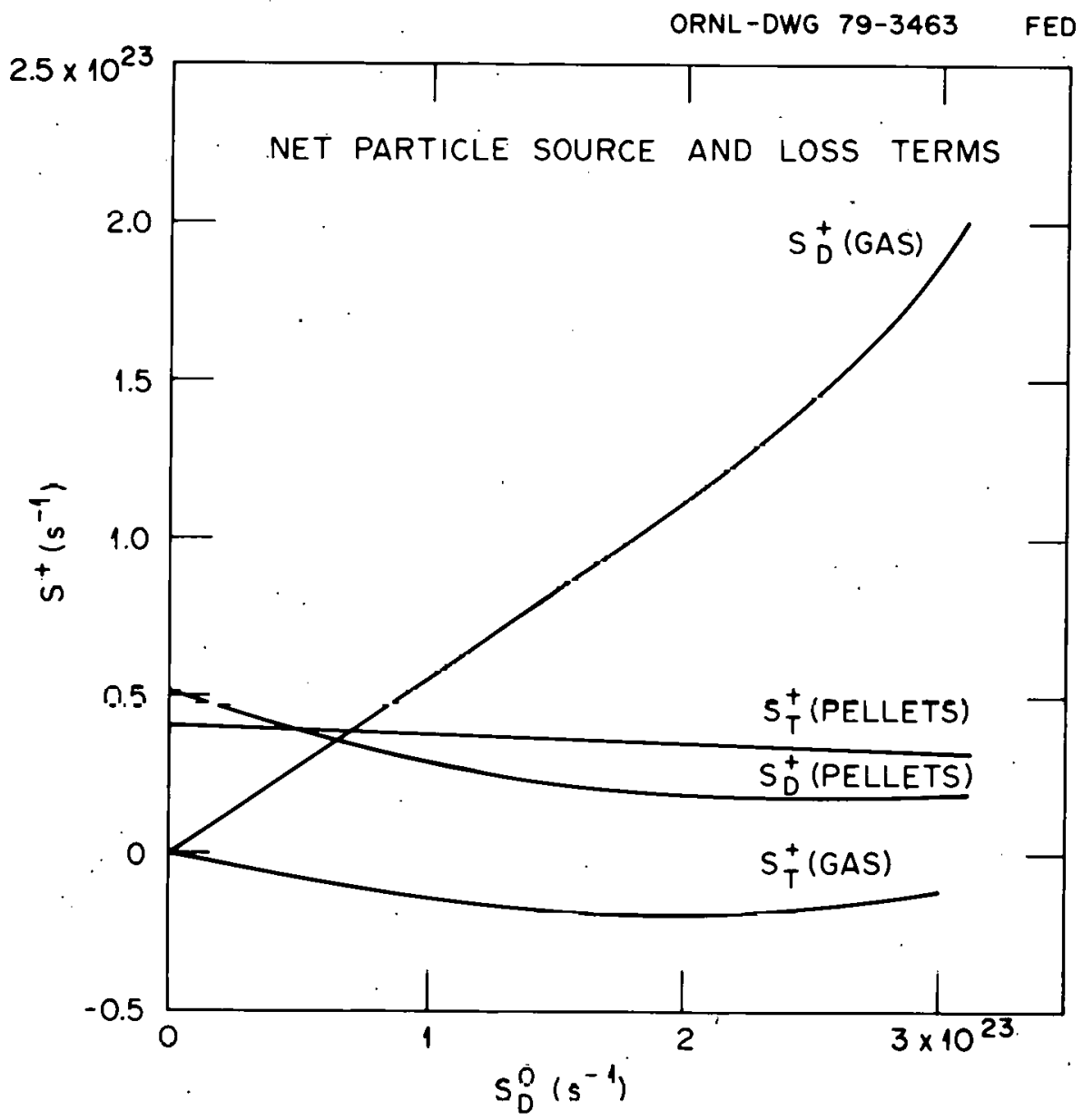

Fig. 2.2. The effect of the deuterium gas puffing rate on the deuterium and tritium pellet fueling requirements. 


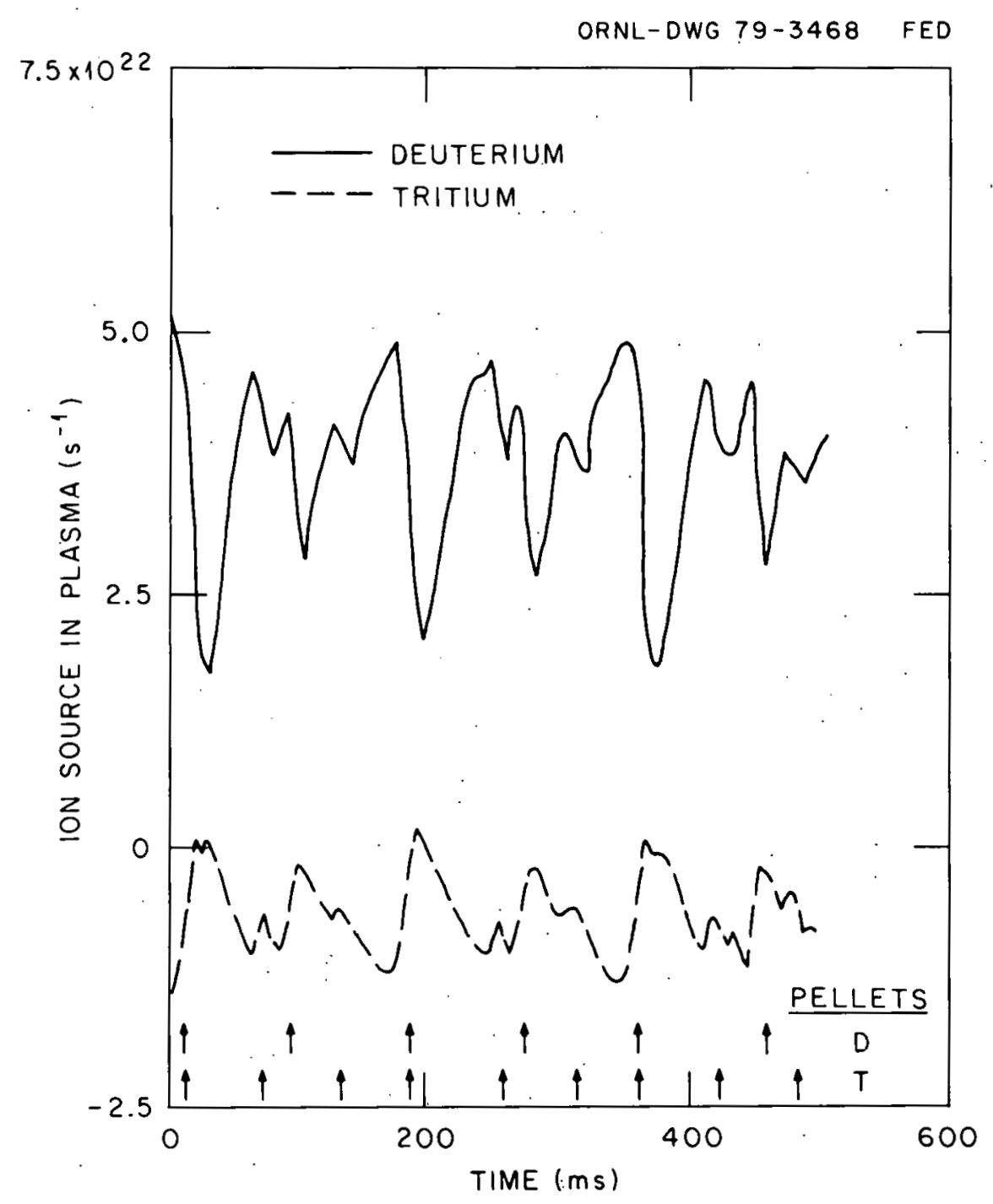

Fig. 2.3. Net particle deposition in the plasma from deuterium gas fluctuates as pellets are injected. There is a net loss of tritium as a result of charge exchange. The deuterium gas feed rate for this example is $1.4 \times 10^{23} \mathrm{~s}^{-1}$. 


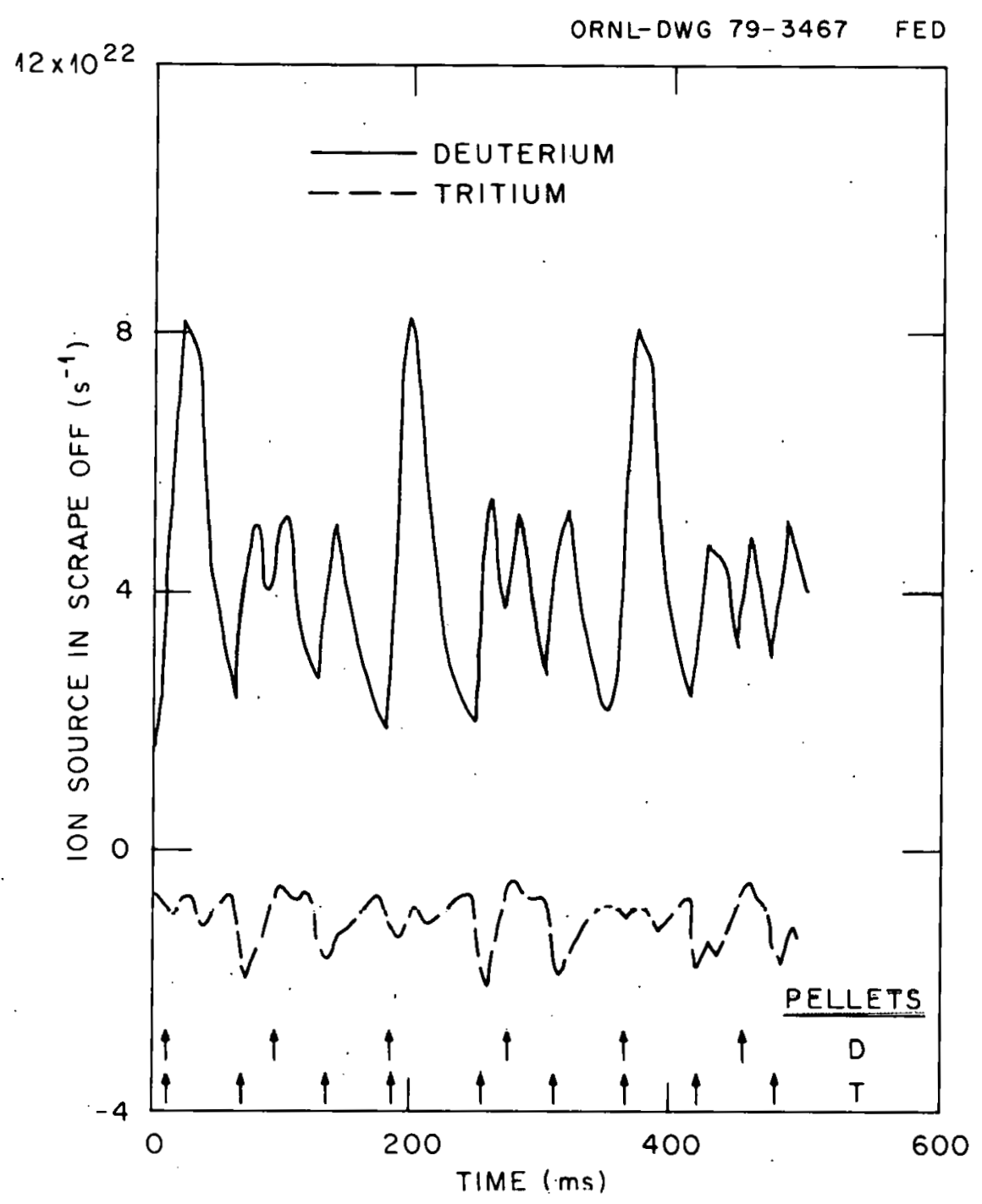

Fig. 2.4. Net particle deposition in the scrapeoff region with a doutcrium gas fced rate of 1.4 ' $x$ $10^{23} \mathrm{~s}^{-1}$. 


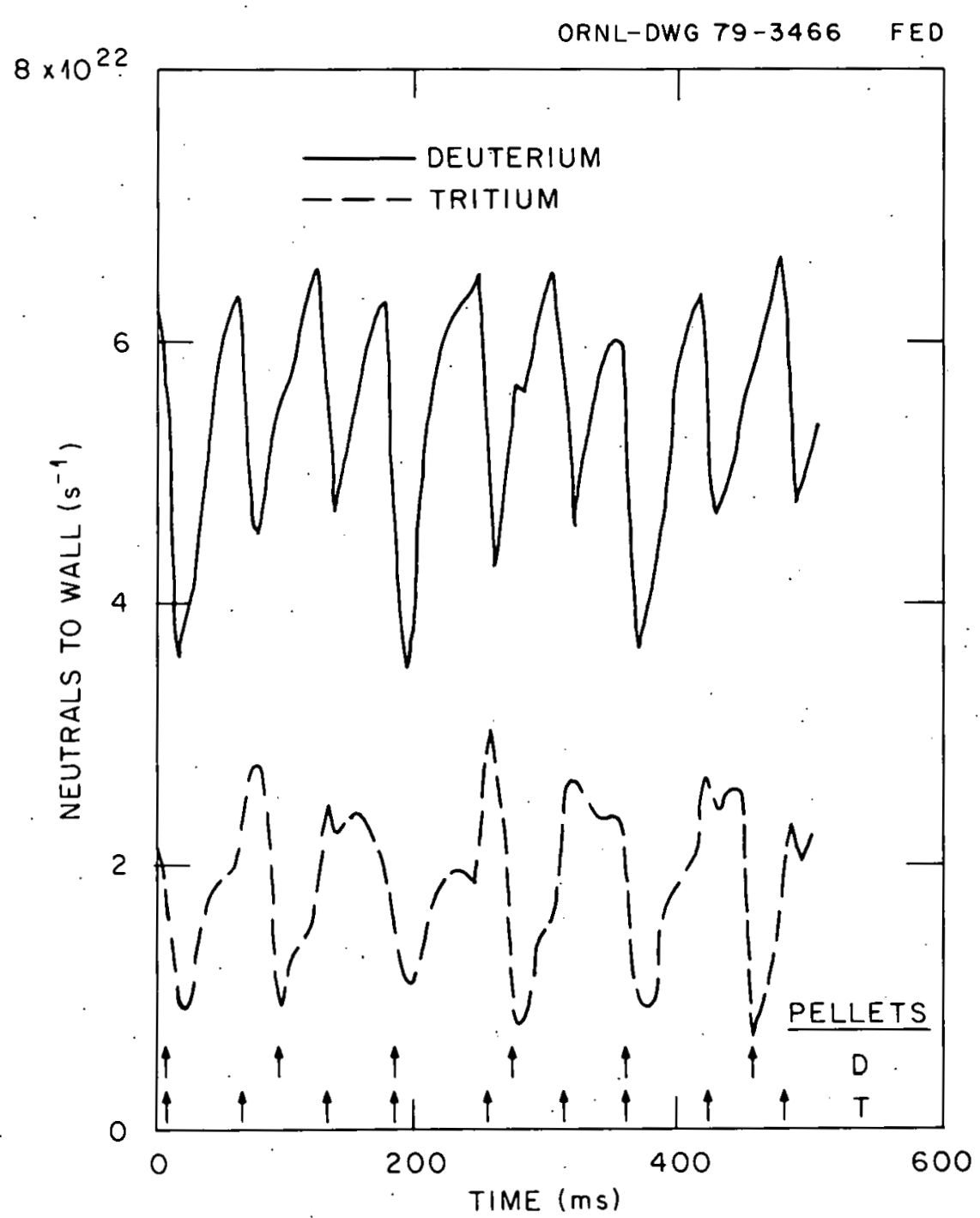

Fig. 2.5. Energetic charge exchange neutral flow rates to the wall for a deuterium gas feed rate of $1.4 \times 10^{23} \mathrm{~s}^{-1}$. 


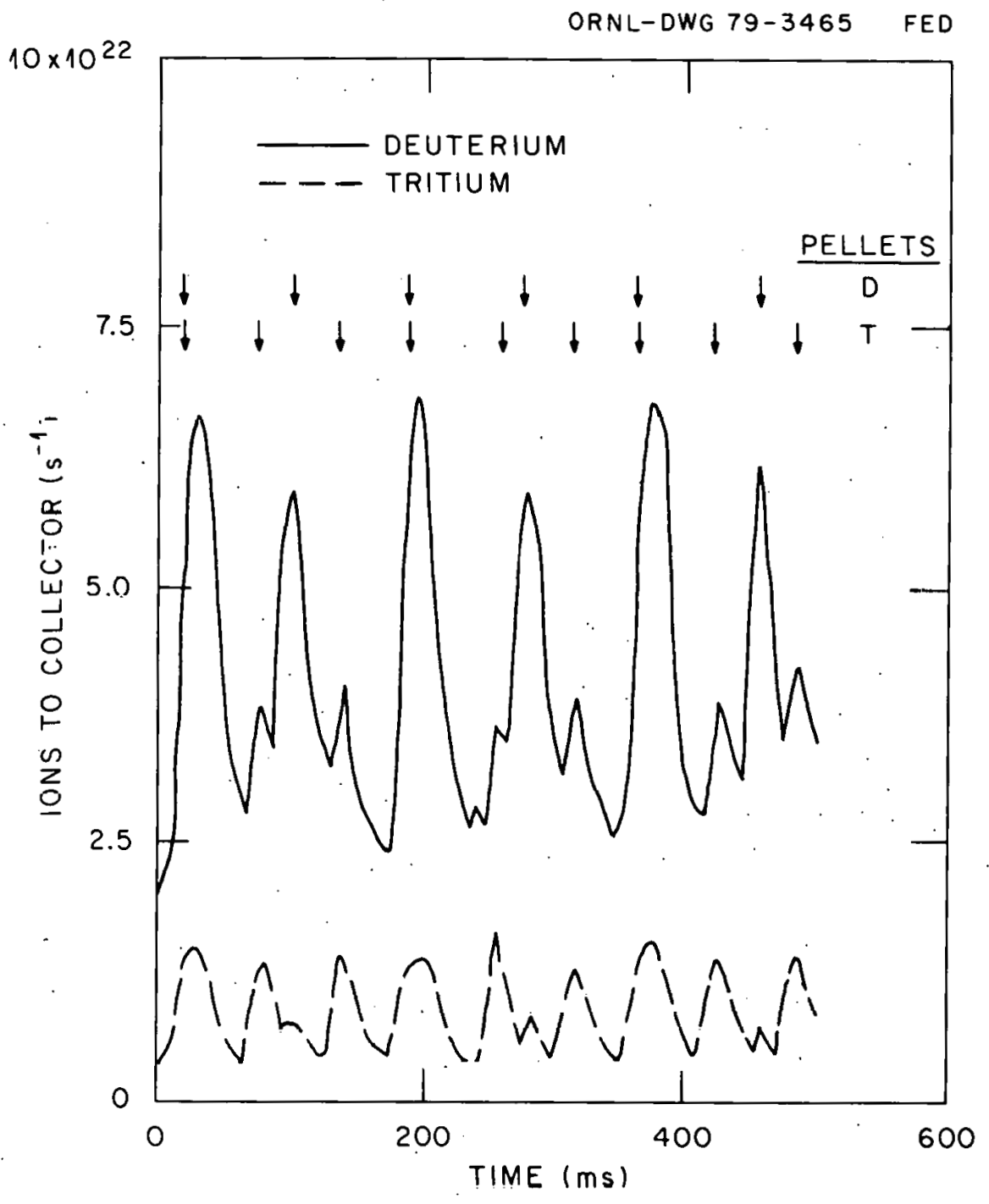

Fig. 2.6. Charged particle flow rates of deuterium and tritilum th the divertor sollector for a doutorium gas feed rate of $1.4 \times 10^{23} \mathrm{~s}^{-1}$. 


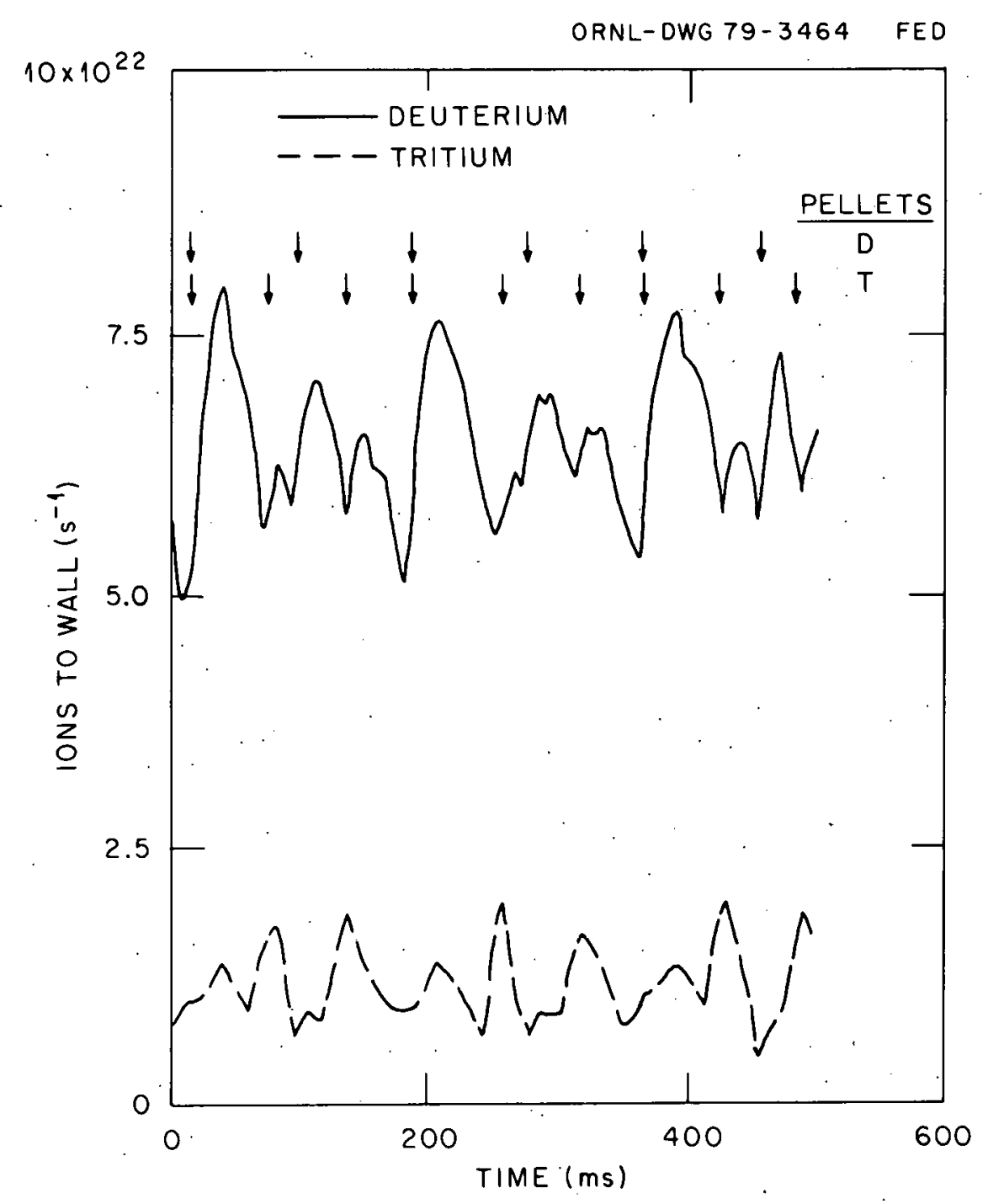

Fig. 2.7. Charged particle flow rates of deuterium and tritium to the wall for a deuterium gas feed rate of $1.4 \times 10^{23} \mathrm{~s}^{-1}$. 
sputtering from the wall and divertor collector plate may alter these results somewhat. Both particle and energy balance calculations must be made to take these features into account in a self-consistent way. The tradeoffs that occur when a poloidal divertor rather than a bundle divertor is used require further analysis. The impact on the results of parallel flow rate, mirroring in the bundle divertor throat, etc., should also be considered. Another area in which the calculations may need refinement is the impact of particle diffusion models on the results in the divertor region as well as in the plasma region. Currently Bohm diffusion is employed in the divertor region, and "PL'T" diffusion is used in the plasma region. Also, an assessment of the sputtering and divertor shielding efficiency is required.

The most important result of this work 1s that little cuntrol on the density of the tritons and deuterons can be exerted by gas puffing through the scrape-off region. Pellet fueling reduces the deuterium and tritium flow to the collector plates. The fluctuations in the operation that are observed may be reduced with the use of smaller pellets and the inclusion of recycle effects from the walls and the collector plate. Thus, it sccms that feedback on pellets for density control is the best approach. This may be achieved by varying the pellet repetition rate, as was done in these calculations, or by varying the pellet size (extrusion rate) for a fixed repetition rate.

\section{REFERENCES}

1. W. A. Houlberg et a1., ORNL/TM-6549, Oak Ridge, Tennessee (1979).

2. W. A. Houlberg and R. W. Conn, Nucl. Sci. Eng. 64, 141-150 (1977).

3. W. A. Houlberg, H. C. Howe, and S. E. Attenberger, ORNL/TM-7124, Oak Ridge, Tennessee (1980).

4. K. Audenaerde, G. A. Emmert, and M. Gordinier, J. Compur. Phys. 34, 268 (1980). 


\section{COMPUTER SIMULATION OF ISX-B PLASMA CURRENT AND HORIZONTAL POSITION CONTROL}

\subsection{SIMULATOR STUDIES OF TOKAMAK CONTROL}

The objective of the hybrid computer modeling program is to explore the dynamic relationships between plasma current and horizontal position vs the action of the field coil current controls. Although the program seeks to develop generic tokamak control algorithms, the ISX-B machine continues to be the system whose characteristics are under study; the existence of an experimental facility makes possible a model-tuning and verification process.

Results of earlier simulations have already been reported; ${ }^{1}$ the model described in this earlier report is the basis for the work presented here (see Table 3.1). In the initial approach control of the plasma current $I_{1}$ (see Table 3.1) was largely neglected, with primary.

Table 3.1. Definition of symbols used in hybrid simulation of ISX-B

\begin{tabular}{|c|c|c|}
\hline Symbol & . Meaning & Unit \\
\hline$I_{1}$ & Plasma current (negative) & $\mathrm{kA}$ \\
\hline $\mathrm{I}_{2}$ & Inner $\mathrm{OH}$ current & $\mathrm{kA}$ \\
\hline $\mathrm{I}_{3}$ & Outer $\mathrm{OH}$ current & $\mathrm{kA}$ \\
\hline $\mathrm{I}_{5}$ & Symmetrical shell eddy current & $\mathrm{kA}$ \\
\hline $\mathrm{B}_{\mathrm{v}}$ & Vertical field & \\
\hline$\beta_{p}$ & Poloidal pressure ratio & \\
\hline$\Delta \beta$ & Change in $\beta_{p}$ & \\
\hline $\mathrm{R}_{1}$ & Plasma major radius & m \\
\hline$\delta R_{1}$ & Change in plasma major radius & $\mathrm{m}$ \\
\hline $\mathrm{V}_{2}$ & $\begin{array}{l}\text { Control voltage on inner magnet } \\
\text { power supply primarily controlling } \\
\text { plasma current } I_{1}\end{array}$ & $\mathrm{v}$ \\
\hline $\mathrm{v}_{3}$ & $\begin{array}{l}\text { Control voltage on outer magnet } \\
\text { power supply primarily controlling. } \\
R_{I}\end{array}$ & V \\
\hline
\end{tabular}


attention paid to the horizontal position $R_{1}$. When the design basis control system was applied to $I_{1}$ as well as to $R_{1}$, the combined effects caused loss of control. This behavior is a function of the very high gain of the overall system because reducing the feedback coupling between $\mathrm{I}_{1}$ and $\mathrm{I}_{2}$ provides good stabilization. Inasmuch as this coupling is a parameter of ISX-B design, it is not a variable at our disposal for system tuning or experimental verification. The same improvement in controllability can be achieved, however, by increasing the gain of the $\mathrm{V}_{2}$ control system by a large factor $(\cong 25 \times$ nominal). With this very high gain $R_{1}$ and $I_{1}$ are well controlled, even for $9 \%$ step increases (or decreases) in the value of $\beta_{p}$. This response ls shown In Flg. 3.1. At this point the system's control action is derived from rate errors only; for long-term stability proportional control terms must he included. A small amount of proportiona1, $I_{1}$ error signal - about $10 \%$ of the nominal value - can be added without losing control, although the noise level in the field currents increases greatly, an indication of approaching loss of equilibrium. On the other hand, even a sma11 amount of proportional $R_{1}$ error signal added to the controlled variables leads to a complete loss of stability. At the time control is lost $V_{2}$ is attempting to go negative, a polarity denied by ISX-B control system design. If the simulation is altered to permit negative $v_{2}$ excursions, $R_{1}$ and $I_{1}$ are Initiaily well controlled, but the field currents and control voltages oscillate with diverging envelopes until control is lost in $0.5 \mathrm{~s}$. Fig. 3.2 illustrates this behavior. Reducing $\Delta \mathrm{R}_{1}$ and $\int \Delta R_{1}$ inputs makes the system stabler but yields a slightly offset $R_{1}$ value. Because the amplifier for the simulated outer field reference current runs in overload in this model, $\int \Delta R_{l}$ is actually not meaningfully implemented.

In all of the foregoing control configurations, simplification in model development inadvertently introduces $R_{1}$ and $I_{1}$ reference levels not only into the proportional but also into the rate error signals. With this shortcoming corrected, an improper ambiguity in set points is removed and the circuit is somewhat stabler. Were it not for a slight residual drift in $R_{1}$, control would be excellent. 


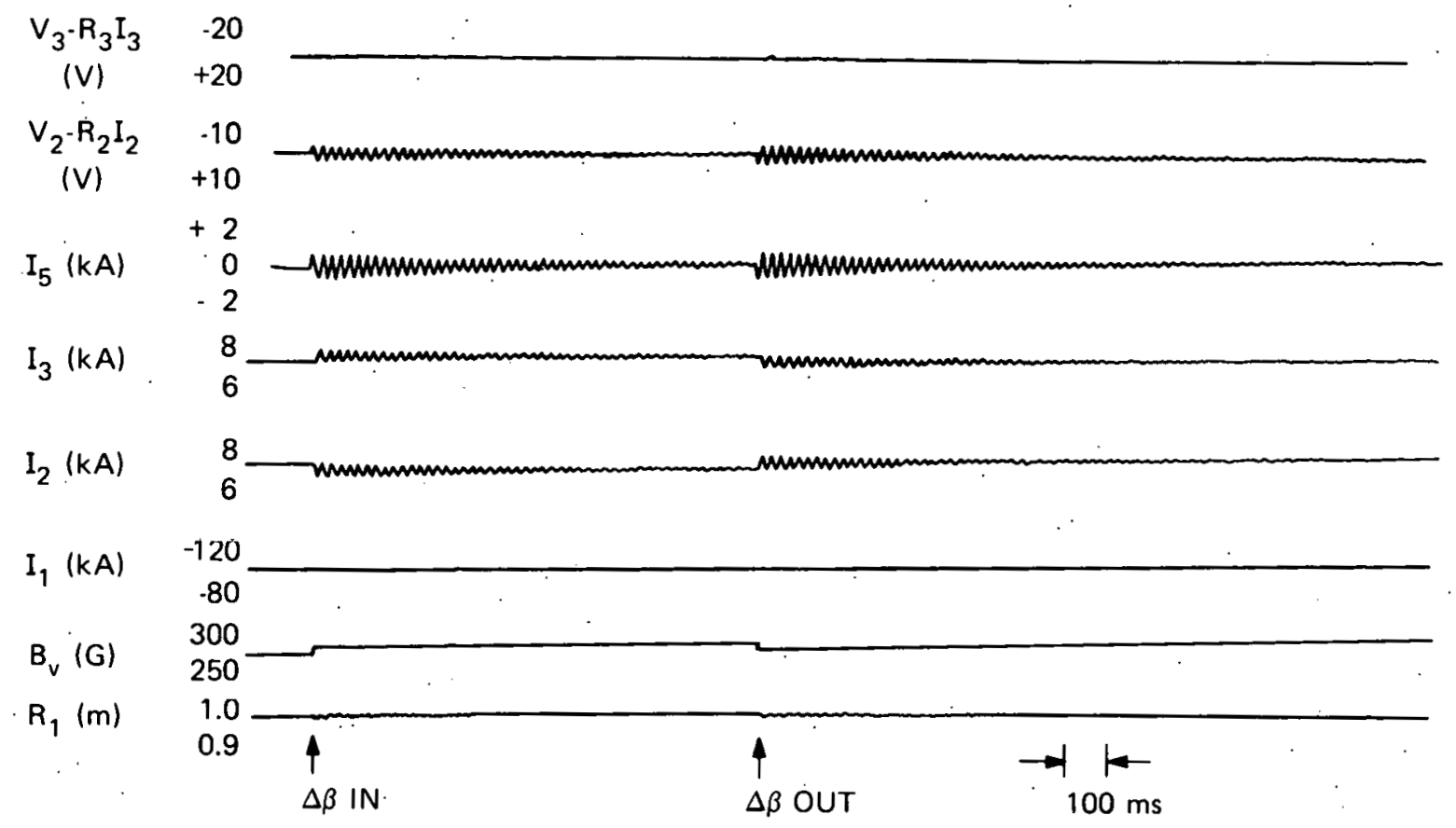

Fig. 3.1. Rate control only; high-gain system. 


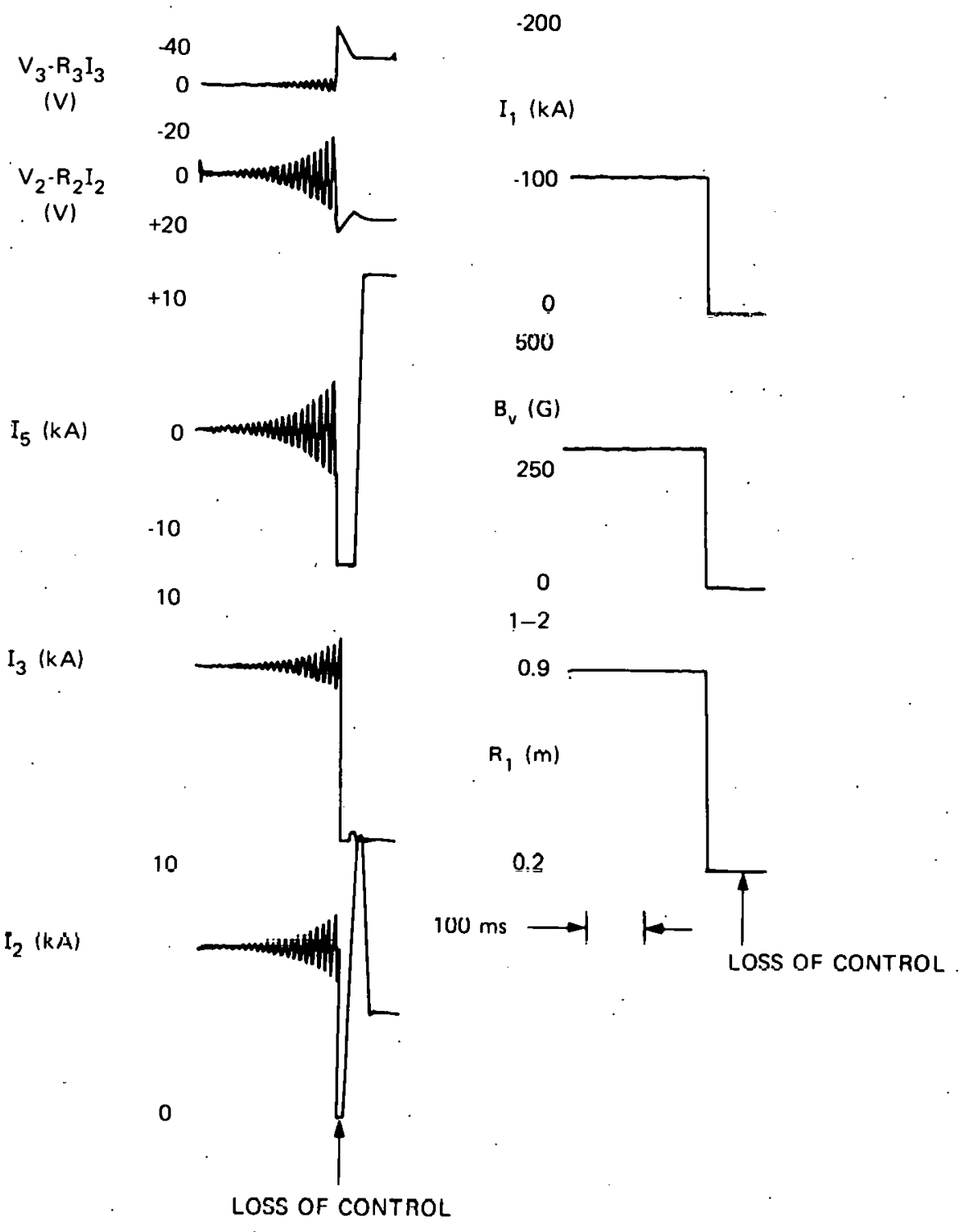

F1g. 3.2. Control w1th $v_{2}$ permiticd negative excursions. 
Where oscillations in the simulated system are encountered, frequencies are $90-100 \mathrm{~Hz}$ (Figs. 3.1 and 3.2). Experimental results in ISX-B show oscillations occurring at $\sim 150 \mathrm{~Hz}$ (Fig. 3.3). Figure 3.3 also shows that as stability is lost, $I_{2}$ and $I_{3}$ diverge, a behavior seen in some of the simulator runs (Fig, 3.4). Thus, the simulator and the experimental machine have similar dynamic characteristics.

There is one stabilizing effect in the experiment that is not included in the model: as $R_{l}$ diverges from its design point, the plasma tends to impinge on the wall, losing current-carrying elements from.its outer edge. A simple version of this behavior was programmed into the simulation. Under its terms $R_{1}$ could not deviate from its design point by large amounts; attempts to do so resulted in progressively greater attenuation of $I_{1}$, representing plasma loss to the wall. This circuit proved to have very little effect on system behavior and was removed.

In the field a successful cut-and-try stratagem for improving ISX-B horizontal stability resulted from the cross-coupling of the plasma current and position controls. This procedure prevents electronic squabbling between autonomous control systems; such disagreements can lead to loss of all control when the requirements for $R_{1}$ and. $I_{1}$ provide conflicting field current commands. The equations used for the two field current set points are

$I_{2 c}=4 \int\left[200\left(I_{1}-I_{10}\right)+\dot{I}_{1}\right] d t+C_{32} \int\left[200\left(R_{1}-R_{10}\right)+\dot{R}_{1}\right] d t$

and

$I_{3 c}=60 \int\left[200\left(R_{1}-R_{10}\right)+\dot{R}_{1}\right] d t+C_{23} \int\left[200\left(I_{1}-I_{10}\right)+\dot{I}_{1}\right] d t$.

In our original control scheme $\mathrm{C}_{32}=\mathrm{C}_{23}=0$. Cross-coupled in the new simulation, $\mathrm{C}_{32}=150 \mathrm{kA} / \mathrm{m}$ and $\mathrm{C}_{23}=0.2 \mathrm{kA} / \mathrm{kA}$. Using these values results in the simulator output shown in Fig. 3.4. Here $R_{1}$ and $I_{1}$ are controlled in a long-term fashion, albeit with $3-6 \%$ wanderings from their set points. 
ORNL-DWG 80-2831 FED

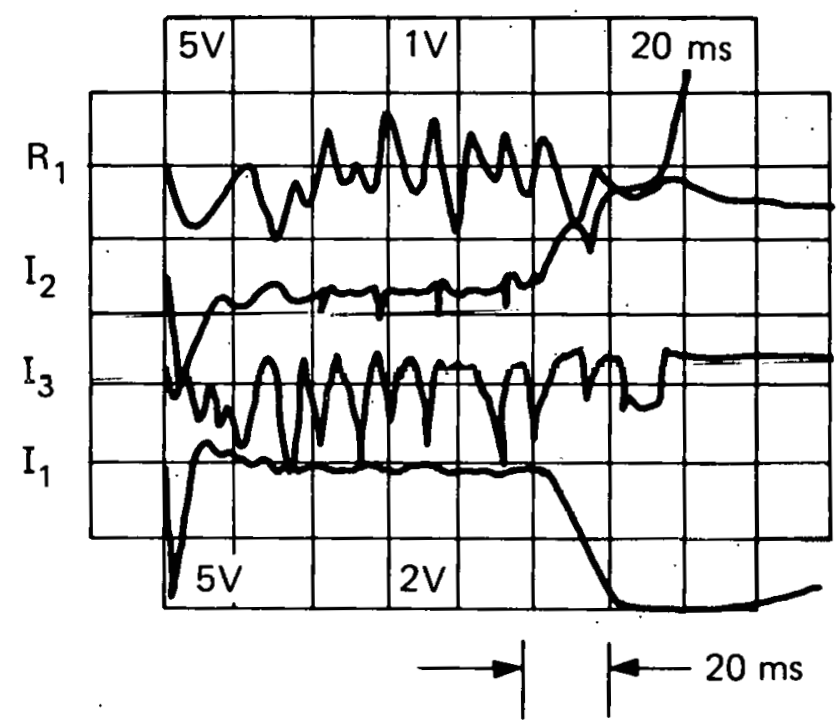

Fig. 3.3. Experimental run on ISX-B. 
ORNL-DWG 80-2832 FED

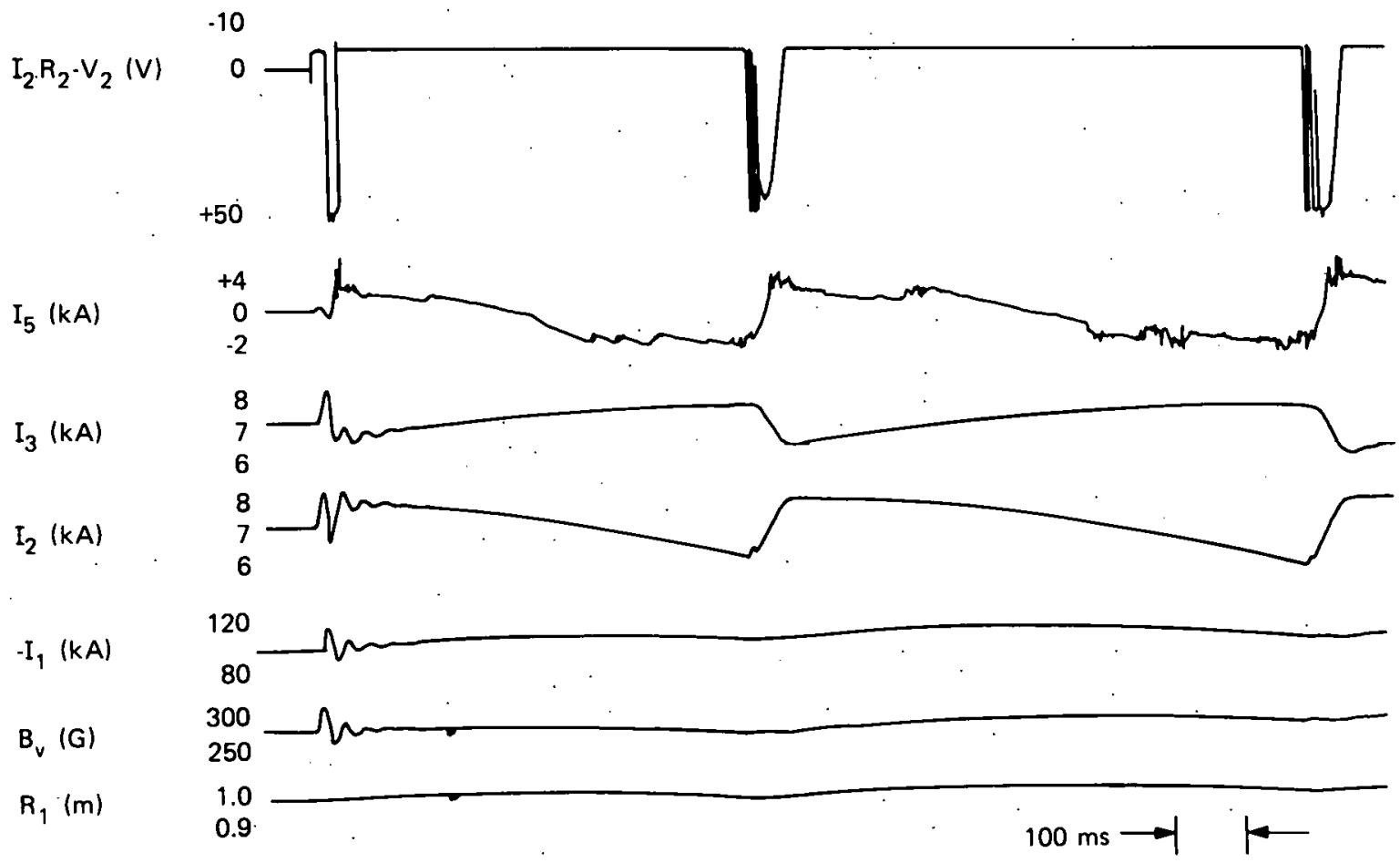

Fig. 3.4. Stable control of $I_{1}$ and $R_{1}$. 
In other control configurations the inner and outer field currents $I_{2}$ and $I_{3}$ have sometimes diverged without limit. In the case shown in Fig. 3.4 these control currents begin such behavior but then recover through periodic control action wherein the field currents rapidly retrace the paths of their divergent drift. During the drift itself calculated $V_{2}$ is negative; in the ISX-B system this means that there is no output from the inner field coil control system. As the intermittent corrective phase begins, $V_{2}$ goes positive and the inner winding control system comes alive briefly. The damped oscillations near the start of the traces represent initial transients excited when the machine is thrown into the RUN mode. By setting initial conditions for $\mathrm{I}_{2}$ and $\mathrm{I}_{3}$ closer to equilibrium values, these initial transients can be eliminated.

The power supply characteristics in the equations used were based on idealized concepts. Actual time-constant delays and nonlinearities should be addressed for more rigorous design studies. In addition, desired plasma elongation will require a curved field and hence better vertical position control through the radial field coils. None of these issues were addressed in the present analysis, which, however, has demonstrated the usefulness of an easily observed and quickly programmed analog control mockup.

\subsection{OBSERVATIONS AND CONCLUSIONS}

The incorporation of the vertical as well as the horizontal position and plasma current into a unified control stratagem will make the system more complicated but may permit plasma shaping to take place. A coupling between the vertical position and the elongation of the plasma requires careful analysis of any proposed control strategy.

The cross-coupling between plasma current control and plasma position that was put into the ISX-B control has quieted the system and made it stabler. Figure 3.4 includes this cross-coupling, but it is absent in Figs. 3.1-3.3. A physical interpretation of all of the oscillations in Fig. 3.3 and the importance (or lack of importance) of including them in the system equations may prove beneficial for later tokamak control systems. 


\section{REFERENCE}

1. W. R. Becraft et a1., ORNL/TM-6617, Oak Ridge, Tennessee (1979). 
24

THIS PAGE

WAS INTENTIONALLY

LEFT BLANK 
4. MULTIVARIABLE FEEDBACK CONTROL SYSTEM DESIGN FOR ISX-B

\subsection{INTRODUCTION}

As indicated in Sect. 3, the controller currently used on ISX-B controls the magnitude and horizontal position of the plasma current by rate and proportional error signals. Proportional control is probably not necessary for pulse duration $<1 \mathrm{~s}$. For longer pulses rate and proportional control will be necessary because some drifting has been observed on the simulations without proportional feedback.

If the control system were to be designed and developed again with our current understanding of the system, a different controller would doubtless emerge. The purpose of this section is to outline the initiation of a new controller design for ISX-B. Figure 4.1 is a block diagram of the system. $I_{1}$ and $R_{1}$ are to be kept within specified limits (see Table 3.1 for notation).

ISX-B control is achieved through a variation of current in the inner and outer poloidal field coils. Because these two control windings are coupled to each other as well as to the plasma, complete separation of their control function is not possible. The inner winding, which primarily controls plasma current, also influences plasma position, and the outer winding affects not only the position but also, the current. This leads to a multiple-input, multiple-output control system.

The design for a multivariable feedback control system from a transfer function matrix viewpoint was chosen even though others ${ }^{1,2}$ have used the state-variable approach. The reasons are as follows.

(1) The design of controllers for plasmas is in its infancy; thus, frequency-domain techniques provide easier methods for hardware design than modern control techniques.

(2) At this stage the specification of a performance criterion may be premature. Once a controller is designed, later state-space techniques may be used to optimize the parameters.

The extended root locus technique is used for actual design. 


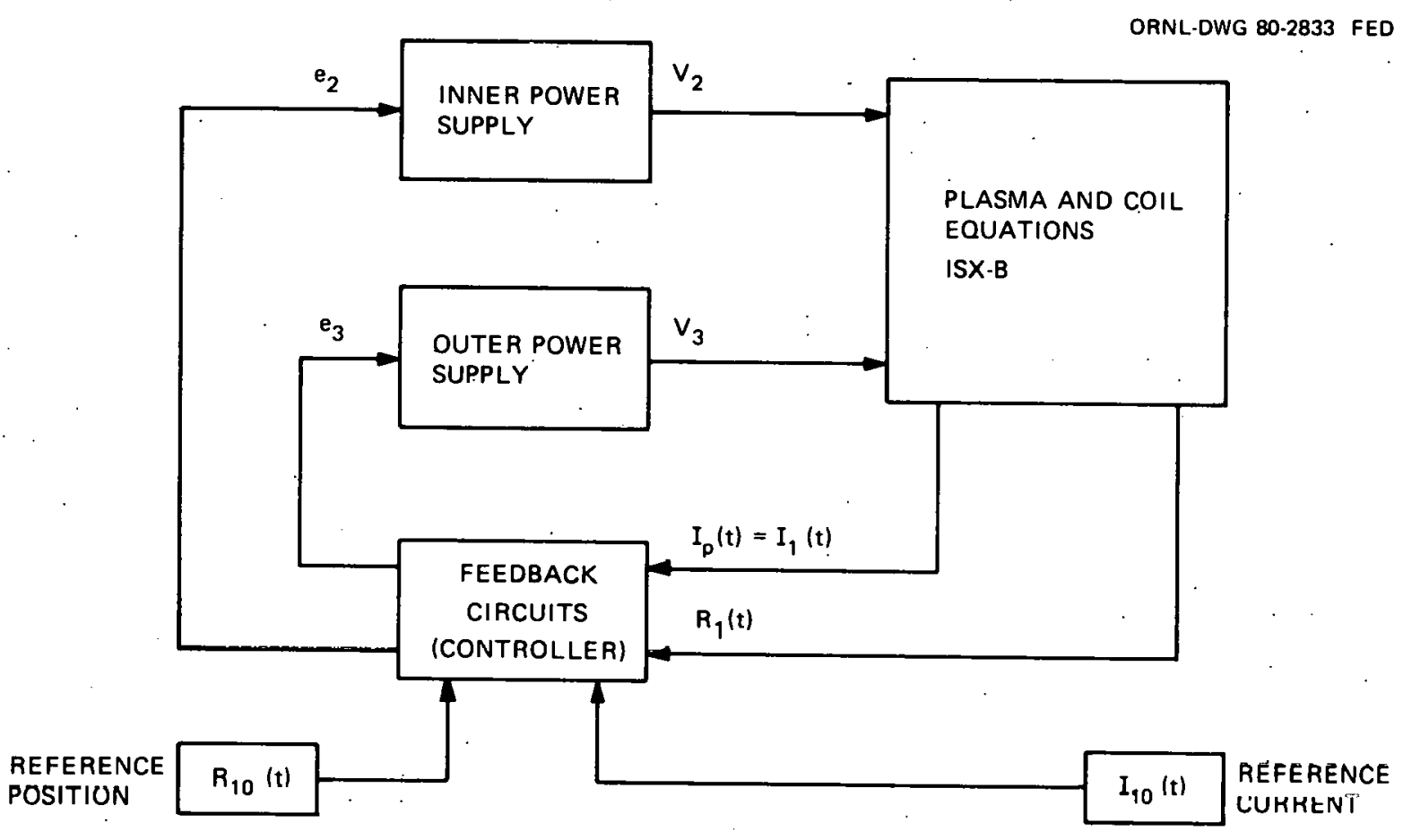

Fig. 4.1. A feedback control for ISX-B. 


\subsection{THE TRANSFER FUNCTION MODEL $G(s)$ FOR ISX-B}

Figures $4.1,4.2$, and 4.3 are block diagrams of the three main components of the system: ISX-B, the controller, and the power supplies. The self- and mutual inductances and initial parameter values are given in Table 4.1. The dynamic equations ${ }^{3}$ for the ISX-B horizontal control are given below:

$\mathrm{L}_{11} \dot{\mathrm{I}}_{1}+\mathrm{L}_{12} \dot{\mathrm{I}}_{2}+\mathrm{L}_{13} \dot{\mathrm{I}}_{3}+\mathrm{L}_{15} \dot{\mathrm{I}}_{5}+\left(\mathrm{L}_{11}^{\prime} \mathrm{I}_{10}+\mathrm{L}_{12}^{\prime} \mathrm{I}_{20}+\mathrm{L}_{13}^{\prime} \mathrm{I}_{30}\right.$

$$
\left.+\dot{L}_{15}^{\prime} I_{50}\right) \dot{R}_{1}+R_{1} I_{1}=0
$$

$\mathrm{L}_{12} \dot{\mathrm{I}}_{1}+\mathrm{L}_{12}^{\prime} \mathrm{I}_{10} \dot{\mathrm{R}}_{1}+\mathrm{L}_{22} \dot{\mathrm{I}}_{2}+\mathrm{L}_{23} \dot{\mathrm{I}}_{3}+\mathrm{L}_{25} \dot{\mathrm{I}}_{5}+\mathrm{R}_{2} \mathrm{I}_{2}=\dot{\mathrm{V}}_{2}$

$\mathrm{L}_{13} \dot{\mathrm{I}}_{1}+\mathrm{L}_{13}^{\prime} \mathrm{I}_{10} \dot{\mathrm{R}}_{1}+\mathrm{L}_{23} \dot{\mathrm{I}}_{2}+\mathrm{L}_{33} \dot{\mathrm{I}}_{3}+\mathrm{L}_{35} \dot{\mathrm{I}}_{5}+\mathrm{I}_{3} \mathrm{R}_{3}=\mathrm{V}_{3}$

$\mathrm{L}_{15} \dot{\mathrm{I}}_{1}+\dot{\mathrm{L}}_{15}^{\prime} \mathrm{I}_{10} \dot{\mathrm{R}}_{1}+\mathrm{L}_{25} \dot{\mathrm{I}}_{2}+\mathrm{L}_{35} \dot{\mathrm{I}}_{3}+\mathrm{L}_{55} \dot{\mathrm{I}}_{5}+\mathrm{I}_{5} \mathrm{R}_{5}=0$, and

$R_{1}(t)=R_{10}+\sum g_{1}\left(I_{1}-I_{10}\right)+\delta R(t)$.

These equations are linear with constant coefficients. This means that the inductances $L_{i j}$ are assumed to be independent of plasma current and position.

A transfer function for ISX-B is obtained by taking the Laplace transform of Eqs.(4.1)-(4.5) with zero initial conditions and arranging them in the form.

$$
\begin{aligned}
& a_{11} I_{1}(s)+a_{12} I_{2}(s)+a_{13} I_{3}(s)+a_{15} I_{5}(s)+a_{16} R_{1}(s)=0, \\
& a_{21} I_{1}(s)+a_{22} I_{2}(s)+a_{23} I_{3}(s)+a_{25} I_{5}(s)+a_{16} K_{1}(s)=v_{2}, \\
& a_{31} I_{1}(s)+a_{32} I_{2}(s)+a_{33} I_{3}(s)+a_{35} I_{5}(s)+a_{36} R_{1}(s)=v_{3} \text {, } \\
& a_{41} I_{1}(s)+a_{42} I_{2}(s)+a_{43} I_{3}(s)+a_{45} I_{5}(s)+a_{46} R_{1}(s)=0 \text {, and }
\end{aligned}
$$




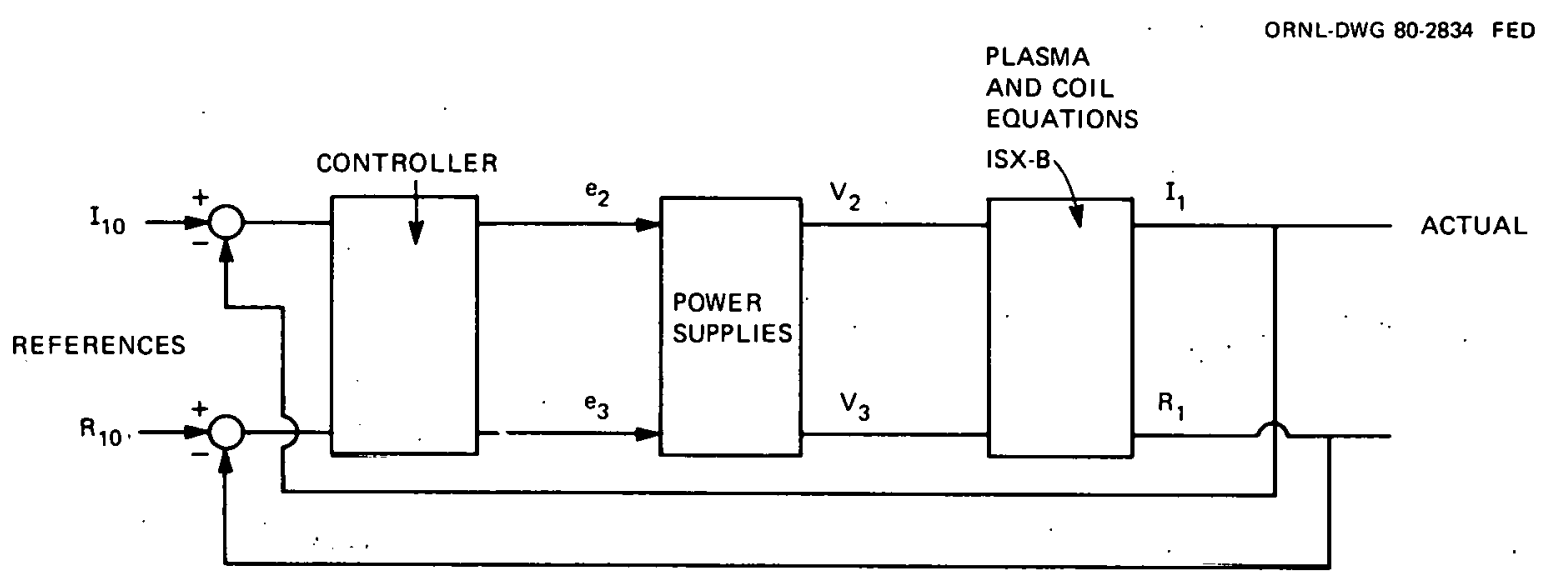

Fig. 4.2. An equivalent model of Fig. 4.1. 


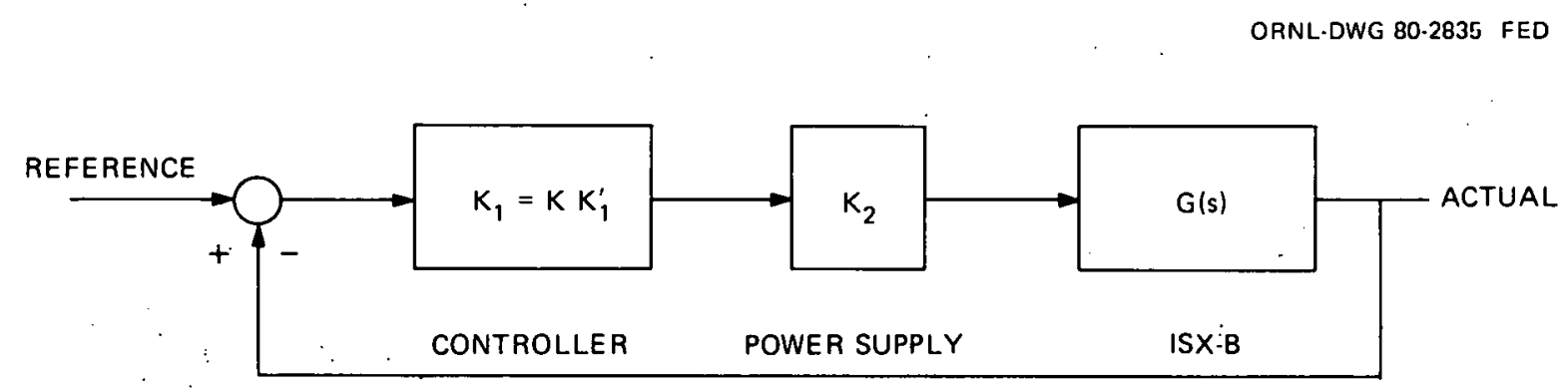

Fig. 4.3. An equivalent model of Figs. 4.1 and 4.2. 
Table 4.1. Parameter values for ISX-B controller design

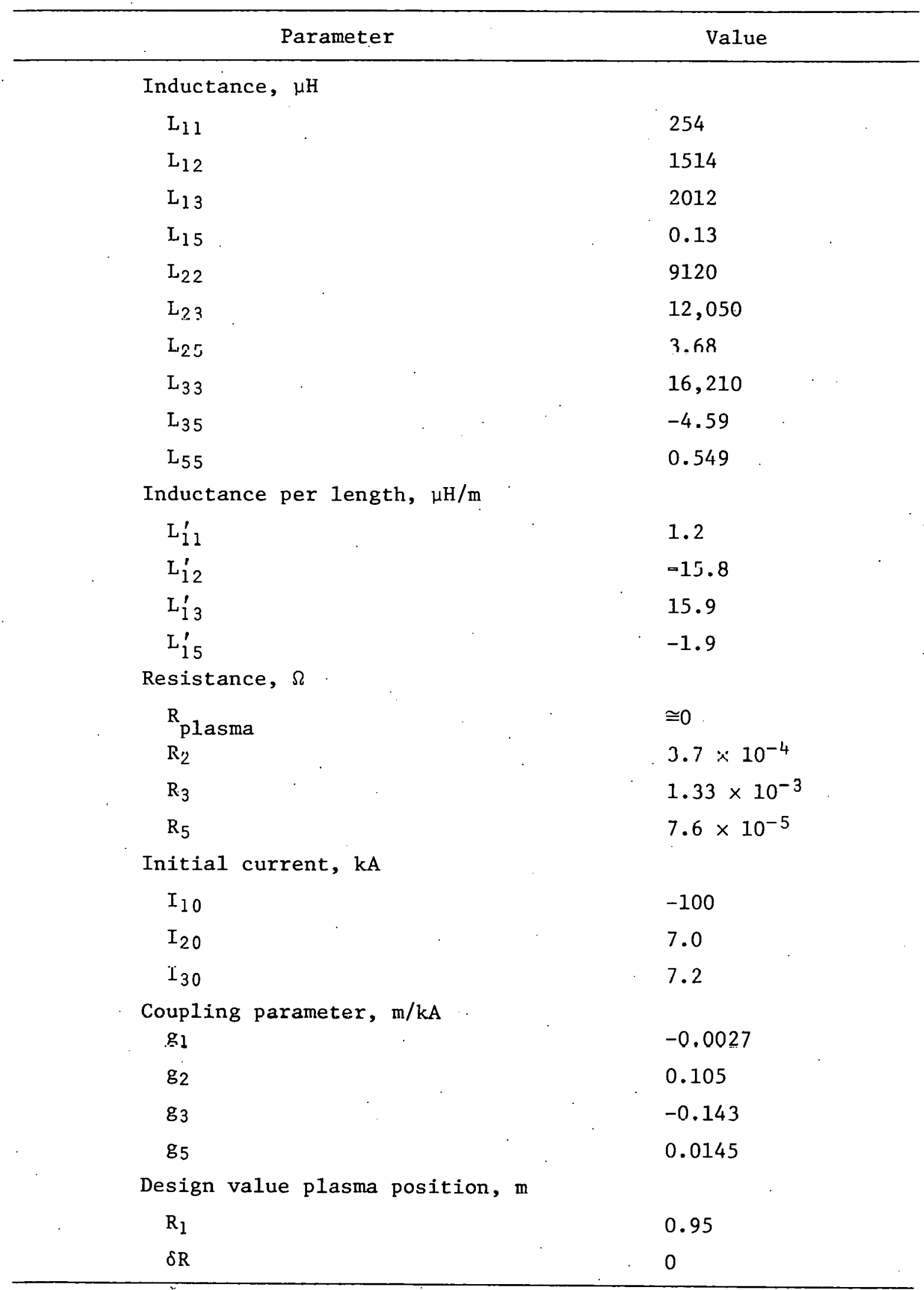


$a_{51} I_{1}(s)+a_{52} I_{2}(s)+a_{53} I_{3}(s)+a_{55} I_{5}(s)+a_{56} R_{1}(s)=0$,

where all $a_{i j}(i=1,2,3,4,5 ; J=1,2,3,5,6)$ are constants.

The controller is to be designed so that the system response to slip changes in $I_{10}$ and $R_{10}$ remains within specified limits. This may be accomplished by the extended root locus method if the transfer function is obtained. Therefore, we value Eqs. (4.6)-(4.10) for $I_{1}$ and $R_{1}$ as functions of $v_{2}$ and $v_{3}$ to obtain

$$
\left[\begin{array}{c}
I_{1} \\
R_{1}
\end{array}\right]=\left[\begin{array}{ll}
G_{11}(s) & G_{12}(s) \\
G_{21}(s) & G_{22}(s)
\end{array}\right]\left[\begin{array}{c}
V_{2}(s) \\
V_{3}(s)
\end{array}\right],
$$

where

$$
\begin{aligned}
& G_{11}=\frac{N_{11}}{\Delta}, G_{12}=\frac{N_{12}}{\Delta}, G_{21}=\frac{N_{21}}{\Delta} \text {, and } G_{22}=\frac{N_{22}}{\Delta}, \\
& G_{11}(s)=\frac{N_{11}}{\Delta} \\
& =\frac{(s+3.08)(s+6.19)(s+6.91)(s+7.15)(s+106.8)}{(s+0.95)(s+3.91)(s+6.18)(s+7)(s+7.07)(s+281)} \\
& N_{12}=(s+0.68)(s+0.17)(s+7.04 \pm j 0.11)(s+636) ; \\
& N_{21}=(s+4)(s+6.16 \pm j 0.72)(s+7.98)(s+772) \text { and } \\
& N_{22}=(s+2.44)(s+6.39 \pm j 0.2)(s+7.49)(s+1507) \text {. }
\end{aligned}
$$

Using the above transfer function for ISX-B, a controller is being designed. A block diagram form of the transfer function is shown in Fig: 4.4 . 
ORNL-DWG 80-2836 FED

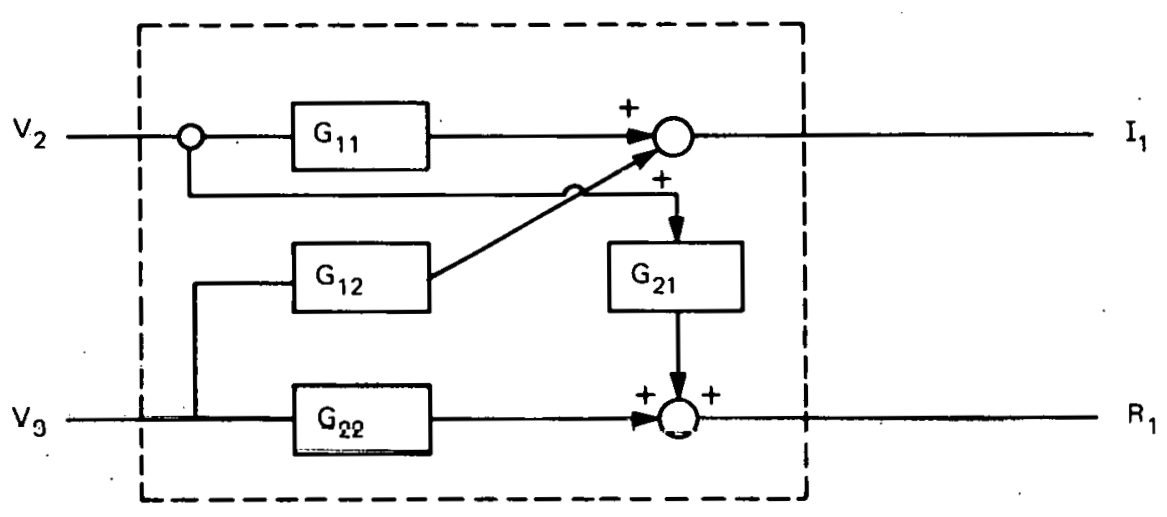

Fig. 4.4. Transfer function mode1 $G(s)$. 
4.3. THE EXTENDED ROOT LOCUS METHOD

The system shown in Fig. 4.3 has a characteristic equation given by

$\left|I+K_{1} K_{2} G\right|=0$.

Let $\mathrm{K}_{1}=\mathrm{KK}_{1}^{\prime}$, where $\mathrm{K}$ is a scalar gain of the controller. Equation $(4.17)$ can be written in the form

$\mathrm{K}^{2} \mathrm{~A} \frac{{ }_{\pi}^{\mathrm{a}}(s-\mathrm{zr})}{{ }_{\pi}(s-P r)}+\mathrm{KB} \frac{{ }_{\pi}^{\mathrm{c}}(s-\mathrm{zt})}{{ }_{\pi}(s-p t)}+1=0$,

where $\mathrm{x}$ denotes the product of $\mathrm{x}$ factors.

A root locus technique is a plot of roots of the characteristic equation. In the single-variable case, the characteristic equation is linear in $\mathrm{K}$; thus, the $\mathrm{K}^{2}$ term is zero.

In the case of ISX-B the controller design is multivariable, and the characteristic equation is quadratic in $\mathrm{K}$ as in Eq. (4.18). The root locus procedure is complex, and a computer program is being developed to plot the root locus for varying $\mathrm{K}$. Once such a program is developed, one can design a variety of controllers with different configurations and parameters, using the standard root loçus techniques available in the literature.

\subsection{SUMMARY}

The characteristic equation that was developed [Eq. (4.18)] will be used to design a number of different controllers to see which concept is best for ISX-B control. A computer program for the solution of Eq. (4.18) is belng written. After this is accomplished, responses to step inputs in $R_{10}, I_{10}$, and $\delta R(t)$ will be observed. Also, stability and controllability to cross-coupling will be observed. 


\section{REFERENCES}

1. J. N. Brooks and R. L. Kustom, ANL/RPP/TM-119, Argonne, Illinois (1979).

2. T. N. Edelbaum, P. A. Madden, and R. E. Var, EPRI ER-533, Charles Drake Laboratory, Inc., Cambridge, Massachusetts (1977).

3. W. R. Becraft et al., ORNL/TM-6617, Oak Ridge, Tennessee (1979). 


\section{MAJOR PLASMA DISRUPTIONS}

\subsection{THE POSSIBILITY OF CONTROL OF AN INCIPIENT MAJOR PLASMA DISRUPTION}

An interaction of the $2 / 1$ and $3 / 2$ modes of the helical flux function is apparently responsible for major plasma disruptions (MPD's). Three time periods exist ${ }^{1}$ that correspond to deformations in the current density profile: (1) before the disruption (the initial phase), (2) during the transition period (where these modes interact), and (3) during the disruption itself. To prevent the disruption one can prevent the growth of either the $3 / 2$ or the $2 / 1$ mode (or both). so that overlap does not occur. The $2 / 1$ mode, centered at a large radius, is more accessible to control by pellet injection or neutral beam heating. Also, the growth rate of the $2 / 1$ phase is slower; therefore, it seems reasonable to control this mode to control disruptions.

Feedback stabilization has been studied theoretically ${ }^{2}$ by switching on and off a feedback signal when the $2 / 1$ mode and the feedback signal are locked in phase at the limiter. Stabilization might thus be achieved experimentally by using a helically wound coil that is actuated periodically depending upon the $2 / 1$ growth mode.

Another method of stabilizing the plasma is to heat it outside the $q=2 / 1$ surface. As the temperature of the region outside this surface increases, it modifies the current profile near the $q=2$ surface and the growth rate of the $2 / 1$ (and $3 / 2$ ) mode decreases. The disruption is thus avoided.

Both of these methods of stability control depend upon the possibility of detecting a precursor signal to a MPD. If the $q(r)$ profile is modified in the wrong place, destabilization may be enhanced; therefore, good diagnostics with rapid response is necessary. The time delay for the feedback system should be less than $\mathrm{r}_{21}^{-1}$ (the growth rate factor for the $2 / 1$ mode). For the ETF it is estimated that the growth rate factor is $224 \mathrm{~ms}$; thus, the feedback system's time response should be less than $210 \mathrm{~ms}$. 
A suitable precursor signal might be obtained by observing the poloidal field, which is apparently strongly influenced just before a disruption. The translation of the physical ideas into working hardware will require more time and effort.

Because the avoidance of MPD's is not presently available, the probable consequences of disruptions are discussed below for an offcentered plasma in a circular vessel and for a D-shaped plasma in a D-shaped vessel. Appendix E provides calculational details and additional data.

\subsection{SUMMARY OF THE CONSEQUENCES OF A MAJOR PLASMA DTSRUPTION}

If a MPD were to occur in an ETF machine, the following results would be likely. First, we define $\delta$. as the distance between the center of the circular vessel and the magnetic center of the plasma. The $D$-shaped plasma is considered to be one-half of an ellipse with $\varepsilon$ the eccentricity and $\delta_{D}$ " the displacement of the magnetic center of the plasma from the straight-line portion of the wall. The ratio $\delta_{D} / a$ is the ratio of the distance of the magnetic center to the minor radius of the ellipse.

The following conclusions have been derived.

(1) It is likely that the plasma disruption time $\tau_{D}$ will be longer than previously anticipated. Instead of $100 \mu \mathrm{s}$, the disruption time will probably be of the order of milliseconds for the ETF.

(2) The eddy currents are much more important in force considerations, power supply protection, etc., than in contributions to the heat load through $\mathrm{I}^{2} \mathrm{R}$ (Joule) heating: Except for extremely asymmetric configurations, it seems that in most cases $<10 \%$ of the temperature change in the wall will be due to the induced eddy currents.

(3) The temperature $r i s e$ is not very sensitive to the shape of the heat pulse that strikes the wall. The disruption time $\tau_{D}$ and the total thermal energy in the plasma primarily determine the temperature distribution in the wall.

(4) The maximum wall temperature for circular tokamaks (about $1000^{\circ} \mathrm{C}$ for $\delta / \mathrm{a}=0.5$ ) increases as $\delta / \mathrm{a} \rightarrow 1$, and the maximum temperature 
is always in the inside region of the torus. For D-shaped tokamaks the maximum wall temperature increases as $\varepsilon \rightarrow 1$ and decreases as $\delta_{D} \rightarrow 1$. The maximum temperature (about $1100^{\circ} \mathrm{C}$ for $\varepsilon=0.968$ and $\delta_{\mathrm{D}} / \mathrm{a}=0.167$ ) generally occurs in the top portion of the $D$; this is a result that depends on the constant radial velocity assumption.

(5) The maximum number of MPD's before wall failure occurs decreases as $\delta / a \rightarrow 1$, with 48 being the minimum number under any circumstances for the ETF. Because there is more surface area per plasma volume in the D-shaped design this number increases to 60 MPD's for the worst case.

\section{REFERENCES}

1. J. D. Callen et al., ORNL/TM-6564, Oak Ridge, Tennessee (1978).

2. J. A. Holmes et al., ORNL/TM-6707, Oak Ridge, Tennessee (1979). 


\section{THIS PAGE WAS INTENTIONALLY LEFT BLANK}




\section{DIAGNOSTICS SUITABLE FOR FEEDBACK CONTROL ON ETF}

\subsection{INTRODUCTION}

Several factors will make the diagnostics for the ETF different than that on existing machines. In addition to the high temperature, neutron fluxes, presence of lithium in the blanket, high magnetic fields, and temperature fluctuations, there can be significant fluctuations of magnetic fields that induce eddy currents in metallic parts and set up force fields. The following factors will need to be taken into consideration when instrumenting the ETF.

(1) Remote maintenance and calibration of the instruments will be required.

(2) The neutron fluxes wili probably prevent direct viewing of the plasma through ports. Some type of mirrored surface for laser light, etc., will probably be necessary.

(3) Because solid-state detectors suffer neutron damage, they probably cannot directly view the plasma.

(4) Magnetic field pickup coils will be located behind the first wall or limiter; thus, a magnetic field diffusion may cause a significant discrepancy between the time something occurs and the time it is measured.

(5) Significant numerical analysis for the rapid interpretation of results (for example, the inversion of data from soft $x$ rays) will be required. Vast amounts of computer interfacing with instrumentation will be necessary.

(6) For some parameters data describing not only the time behavior of a quantity but also the spatial variation will probably be required.

(7) It is probably necessary to be able to measure the deuteron, triton, and electron densities for adequate control because both tritium and deuterium pellec infectors are $11 \mathrm{kely}$ to be required.

(8) The detection of tritium in the presence of background gamma radiation in the structure will be required. Process control, fuel handling, divertor collectors, and lithium blanket processing will involve large amounts of tritium. 
(9) A direct ion temperature measurement would be beneficial. The fusion power production is directly dependent on the ion temperature through $<\sigma v>$.

The diagnostics for the ETF needs a fair amount of attention. In addition, the numerical methods associated with interpreting the outputs of the devices also require work in order to reduce the amount of data and computer storage required. The calculations will have to be done so that on-line computer decisions can be made.

\subsection{TIME CONSTANT TNVOLVED IN DIAGNOETICS AND CONTRULS}

\subsubsection{Major Plasma Disruptions}

The control of incipient MPD's will require diagnostics, computational time, and control action, all completed in a few milliseconds. It may be that arrays of soft $x$-ray detectors provide the most accurate way of determining the probability of an incipient disruption. Even though the time constants associated with solid-state detectors are short (m1croseconds), the conversion of the intensities of the $x$ rays. into a meaningful plasma profile requires computer analysis.

The amount of computational time required to obtain information on the plaswa profile depends on the number of detectors, the computing. system, the interfacing, and the numerical algorithms involved. Assuming that this can be done in a few milliseconds, a control action is required to alter the plasma distribution so that the disruption is averted.

There are at least two ways that control action may be taken (see Sect. 5). The first is to use helically wound feedback coils so that the $\mathrm{m} / \mathrm{n}=2 / 1$ mode is nonlinearly stabiliz.ed. Because this mode rotates with some frequency (approximately drift wave frcquency), the response time for the coil to be activated and the field to penetrate the vessel is roughly the reciprocal of the drift frequency. The feedback signal must be in phase with the $2 / 1$ mode or accelerated destabilization may occur. The other method is to add heat to the plasma at the right place and time. The importance of time constants is apparent in the control of disruptive instabilities. 


\subsubsection{Power Leve1 Contro1}

The power during the burn phase must be controlled in some way. In a fission reactor, control rod positions determine the neutron population. The corresponding control in a fusion reactor may be a ripple coil. The energy multiplication factor ${ }^{l} k_{\text {en }}$ must be kept near unity for fusion control. The fueling rate and species are, of course, also important in power level control.

The important factor from a controls point of view is that for a $D-T$ reactor of $10 \leqslant T_{i} \leqslant .100 \mathrm{keV}$,

$$
\frac{1}{k_{e n}} \frac{d k e n}{d t}>0
$$

This means that the control system will have to be able to respond with time constants less than the energy confinement time $\tau_{E}$. For the ETF $\tau_{E}$ will be $\sim 1.2 \mathrm{~s}$; therefore, it seems that the time constants involved in power control and diagnostics and computational time will not be difficult to obtain. However, the instability of the power level means that nondrifting control systems will be very important.

\subsubsection{Position and Shape Control}

The time constants involved in the measurement, determination, and control action of the plasma position and shape will be of the order of a few milliseconds. Diagnostics can be done via coils or soft $x$-ray detectors, and considerations similar to those that apply to the control of MPD's apply here as well. It is expected that times of a few milliseconds will be required for position and shape control.

\section{REFERENCE}

1. J. Rand McNa1ly, Jr., Nucl. Fusion 17, 1273 (1977). 
7. ASSESSMENT OF THE CONTROLS R\&D NEEDS

\subsection{INTRODUCTION}

The lack of precision in defining the controls $R \& D$ needs reflects the uncertainty in the physics, design, and mission of the ETF. The I\&C design is especially difficult because of the interface that must exist between the hardware and the plasma. The plasma physics is not understood well enough. to make it easy to specify the consequences of certain actions (such as the injection of a pellet).

The functionis of Llie I\&C system on the ETF w1ll be threefold:

(1) to provide manual (open-loop), semiautomatic, and fully automatic (closed-10op) control of the ETF through all phases of operation;

(2) to provide automatic shutdown capability in the event of an environmentally, physically, or economically hazardous situation; and

(3) to provide diagnostic outputs so that important physical parameters may be discerned.

There are two difficulties that must be overcome if automatic control. of fusion reactors is to become a reality involving more than fust plasma position control. These are

(1) the production of the diagnostics necessary for an adequate measurement of system outputs;

(2) the development of adequate mathematical models that relate the response of the plasma to the inputs (these mathematical models may be derived from the application of basic physics laws or from empirical relations arising from the extrapolation of operating machines).

A great gulf exists between present capability and the requirements for ETF control. The single largest aspect of control that needs attention is the development of adequate mathematical models of the plasma. Various design requirements are listed in Ref. 1. Assuming the hurdles of plasma physics state equations can be overcome, the control system design should reflect reasonableness in scope and safety. 


\subsection{DESIGN REQUIREMENTS}

The design requirements can be categorized in several ways. During each pulse of the tokamak various sensors will have to detect data on which the control of the device hinges. These data will doubtlessly go into an on-line computer that will perform calculations and then activate control knobs. The control system should be able to accommodate the following:

(1) normal shutdown in the event of various system malfunctions,

(2) normal shutdown in the event of a safety hazard such as a tritium leak,

(3) automatic remote calibration and checking of sensors,

(4) fecdback and control of the total power of the burn phase,

(5) normal shutdown if various design parameters (such as first-wall temperature) are exceeded, and

(6) automatic control of plasma position and profile to provide reasonable assurance of plasma stability.

These functions can be performed if the proper parameters are sensed. The spatial and temporal variations of various quantities must be sensed. These include

(1) $T_{i}$ and $T_{e}$ of the plasma,

(2) the temperature of the first wa1.1,

(3) the neutron intensity on the first wall,

(4) the plasma current density,

(5) tritium levels throughout the plants and environs,

(6) the divertor collector plate temperature,

(7) electron, deuteron, and triton densities in the plasmas.

Many of these quantities can be obtained directly or be inferred by measurement techniques already in use. The sensing of deuteron and triton densities is more difficult; Appendix B looks at this problem in more detail.

REFERENCE

1. W. R. Becraft et al., ORNL/TM-6617, Oak Ridge, Tennessee (1979). 
THIS PAGE

\section{WAS INTENTIONALLY LEFT BLANK}


APPENDICES 
THIS PAGE

\section{WAS INTENTIONALLY LEFT BLANK}


APPENDIX A

CONTROL OF A TOKAMAK FUSION REACTOR: AN OVERVIEW

\section{A.1 INTRODUCTION}

The practicality of controlled thermonuclear fusion as a power source is dependent on the ability to control the plasma and thus to obtain a relatively inexpensive net power gain. In 1969 Rose pointed to seven problems in magnetically confined systems that must be solved, the first and perhaps most important of which is directly related to control of the device. ${ }^{1}$ As with most physical systems the whole question of controllability depends on finding a region or point in an appropriate state space where the reactor performs well and on designing a control system that keeps the plasma parameters in the neighborhood of that operating point.

A significant step toward commercial fusion is the ETF, which will begin operation about 1990. This device, expected to be a tokamak, will probably have the parameters ${ }^{2}$ listed in Table A.1. Because the tokamak will also probably be a pulsed device, there will be a preignition, burn, and shutdown phase for each cycle. ${ }^{3}$ Most of our remarks concern this reactor because $i t$ is to be the first large D-T-burning facility constructed.

As shown in Table A.2 the three aspects of the closed-1oop control problem that must be considered are plasma position and shape, stability, and power level. Power control is such that the net output power over a cycle maximizes the salable power. In this discussion we ignore the power produced in the blanket. The three aspects of control are not independent of each other because changing a given plasma parameter may affect stability as well as power level. All process I\&C is also ignored because it is likely to be standard.

Figure A.1 is a block diagram of the plasma, the plasma inputs (control knobs), and the three outputs to be controlled. Each area of control must have at least the following information specified in each phase of the operating cycle: 
Table A.1. Likely ETF reactor parameters

\begin{tabular}{|c|c|}
\hline Plasma major radius, $R$ (m) & 5.0 \\
\hline Plasma elongation, $\delta$ & 1.6 \\
\hline Plasma minor radius, a (m) & 1.2 \\
\hline Plasma volume, $v_{p}\left(m^{3}\right)$ & 230 \\
\hline Plasma current, $I_{p}$ (MA) & 5.0 \\
\hline Neutron wall loading $\left(\mathrm{MW} / \mathrm{m}^{2}\right)$ & 2.4 \\
\hline Total fusion power (MW) & 1140 \\
\hline Fusion power density $\left(\mathrm{MW} / \mathrm{m}^{3}\right)$ & 5.0 \\
\hline Number of TF coils & 12 \\
\hline TF coil vertical bore (m) & 9.9 \\
\hline TF coil horizontal bore (m) & 6.2 \\
\hline TF coil conductor & $\mathrm{Nb}_{3} \mathrm{Sn}$ \\
\hline Field at TF coil, $B_{m}(T)$ & 10.9 \\
\hline Field on axis, $B_{t}(T)$ & 5.3 \\
\hline Steady-state burn time (s) & 500 \\
\hline Total cycle time (s) & 560 \\
\hline Total volt $\div$ seconds & 83 \\
\hline Neutral beam energy ( $\mathrm{keV})$. & 150 \\
\hline Injection time (s) & 6.0 \\
\hline Microwave power (MW) & 1.0 \\
\hline Microwave frequency $(\mathrm{GHz})$ & 120 \\
\hline Fueling & Pellet injection \\
\hline Impurity control & Bundle divertor \\
\hline Shielding & $\begin{array}{l}\text { Stainless steel balls } \\
\text { and borated water }\end{array}$ \\
\hline Vacuum topology & Vacuum building \\
\hline Vacuum pumping & Cryopumps \\
\hline
\end{tabular}


Table A.2. Delineation of the control problem in each phase of the operating cycle

\begin{tabular}{cccc}
\hline & & \multicolumn{3}{c}{ Phase } \\
\cline { 2 - 4 } Type of control & Preignition & Burn & Shutdown \\
\hline Position $^{a}$ & Perhaps & $\mathrm{Y}$ & $\mathrm{Y}$ \\
Stability $^{b}$ & $\mathrm{Y}$ & $\mathrm{Y}$ & $\mathrm{Y}$ \\
Power level & $\mathrm{N}$ & $\mathrm{Y}$ & $\mathrm{N}$ \\
\hline
\end{tabular}

$a_{\text {Major radius and vertical position. }}$

${ }^{b}$ Sawtooth, Mirnov, and major disruptions. 


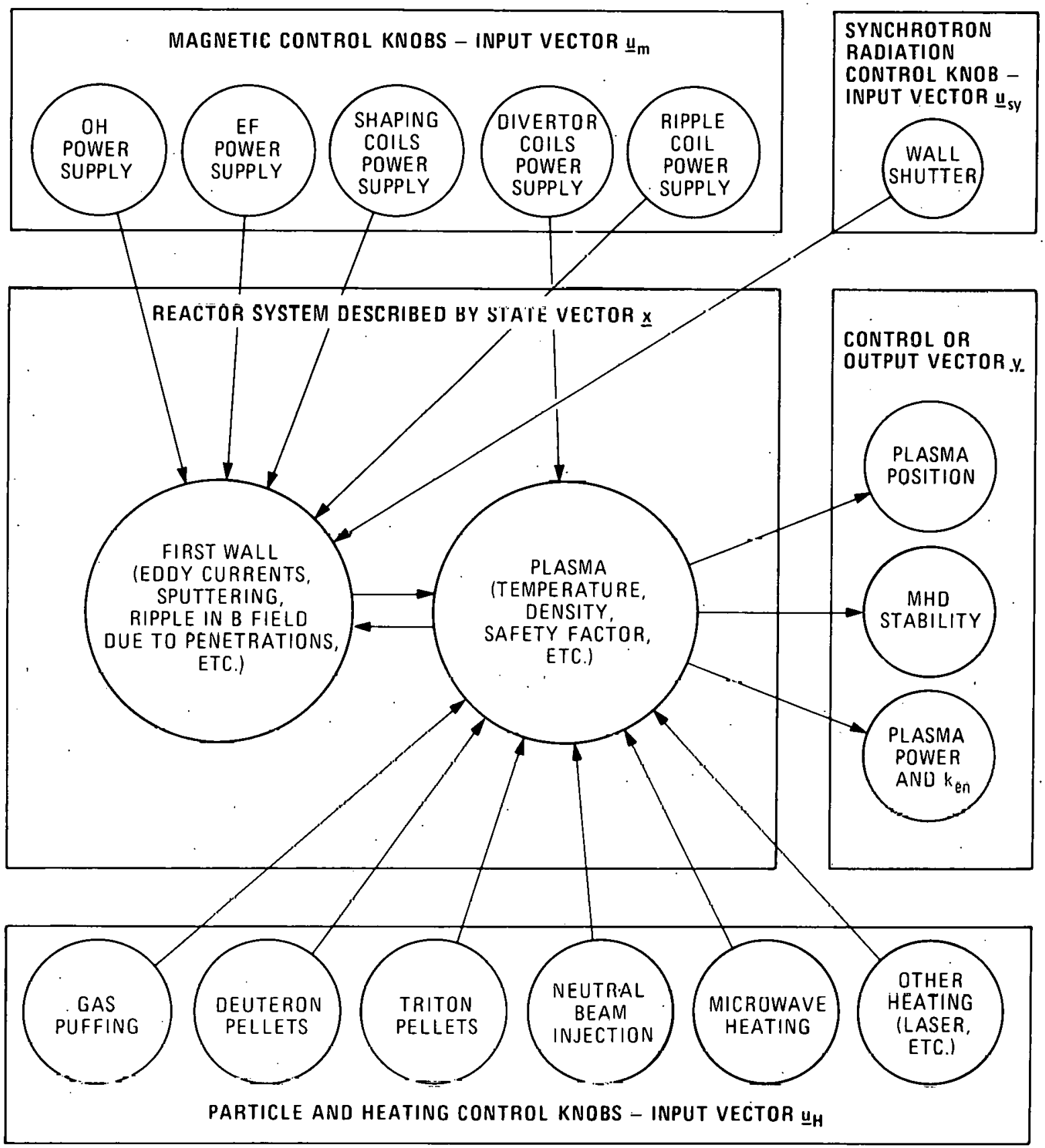

Fig. A.1. Schematic diagram of fusion reactor inputs, outputs, and state variables. 
(1) observable parameters (those quantities that can be detected or directly inferred);

(2) control knobs or input variables;

(3) a system of equations relating the state variables to the input variables and devịce parameters;

(4) an objective function or performance index that is to be achieved by the control system;

(5) relations connecting the observable parameters to the objective function parameters; and

(6) diagnostics used in measuring the observables along with the accuracy and time constants associated with the measurements [these diagnostics must be applicable to the ETF (neutron field, high temperature, intense magnetic fields, large temperature fluctuations) environment].

The purpose of this appendix is to provide an overview of the importance and difficulty of fusion reactor control. Prior to 1970 a great deal of theoretical work was done on various aspects of plasma stability, position, etc. ${ }^{4}$ However, perhaps because of the lack of success in designing hardware with the correct performance capability, the past ten years have not seen as much activity in the area of control. Now, with the designing of large power-producing fusion reactors such as the ETF, a feedback control (closed-loop) system seems imperative. Feedback. control system design cannot merely be a reactor add-on because the control concept is an integral part of the reactor design. This is reflected in pulsed-field effects, first-wall design, coil interconnection approaches, and power supply size, to name a few.

Plasma shape sensing and control will probably have a strong. influence on the stability of the plasma. Disruptive instabilities are sensitive to the safety factor profile $q(r) ;{ }^{5}$ therefore, the separation of plasma.shaping and stability is not likely to be possible. We consider shaping and position together because both are geometrical requirements for the plasma and because the sensing is likely to be done via the same instrumentation.

The development of a state model that consists of a set of differential and algebralc equations connecting the Input vector $\underline{u}$ and the 
state vector $\underline{x}$ is essential. The relationship of the detector outputs $\underline{y}$ to the state variables must also be known (e.g., the relationship of the intensity of the soft $x$-ray detector to electron temperature, densities, etc.). If an adequate model can be developed for the plasma system, then it is probable that a feedback and feedforward (anticipatory) control system may also be designed. We discuss some of the elements of this process in the next sections.

\section{A. 2 STATE VARIABLES AND PERFORMANCE INDICES}

The choice of performance indices and state varlables used to describe the essential physics of the tokamak is not unique. The framework of modern control theory seems to be appropriate for fusion reactor applications because the control system will be a multipleinput, multiple-output device.

Position control is the only aspect that has received any serious attention in a practical sense, ${ }^{6-10}$ although plasma stability has received some attention. ${ }^{5,11}$ Power control is common on fission reactors, but power control for fusion reactors has not received much attention because no net power has yet been produced.

The control knobs or input variables are not yet well defined. Gas puffing, neutral beam heating, pellet infection, ohmic heating, equilibrium field (EF), and shaping coils are all currently being used or are under development. It may be that some mechanism can be used to control the divertor efficiency by changing the divertor coil power supply voltage. A ripple coil may be used to enhance or reduce the ripple. The ripple influences the diffusion of particles, particularly the ions. In Fig. A.1 the inputs are grouped into $\underline{u}_{m}$ (magnetic), $\underline{u}_{h}$ (particle and heating), and $\underline{u}_{s y}$ (synchrotron radiation). Signiflcanc addicional investigations are required to establish the feasibility of synchrotron control.

Synchrotron radiation is reflected from the first wall ( $(90 \%)$ and reabsorbed by the plasma. Exact calculations assoclated with the emission, absorption, and reflection of the radiation from the wall are very difficult, if not impossible, at this time. Because of the high reflection $^{12}$ of the radiation from the wall, it may be that a portion of the 
wall could open (like a window) to allow the synchrotron radiation to go out and be absorbed by anothe: material. However, this sliding opening in the first wall will introduce various complications in the design, thus necessitating a thorough analysis of the concept.

An important point is that $\underline{u}_{h}$ will probably be a much faster-acting input than $\underline{u}_{m}$ or $\underline{u}_{s y}$. The magnetic field diffusion time through the first wall is of the order of tens of milliseconds, and a mechanical slịing shutter will probably require hundreds of milliseconds for travel. Beam heating, microwave heating, gas puffing, etc., will probably be faster by an order of magnitude or so. Therefore, if possible, it may be better to use $\underline{u}_{h}$ for fast control.

The real problem with control of the fusion reactor is a lack of knowledge about the plasma physics in the areas of disruption mechanisms, shutdown phenomena, impurity effects, and divertor physics. Allowable levels of perturbations in density and temperature caused by pellet injection, neutral beams, and impurity buildup need to be established. The state variables and performance indices chosen should be as simple as possible while still allowing for physical constraints such as voltage limitation on power supplies, pellet injection rate, and energy. The formulation of the state equations is not unique. ${ }^{3}$ obtaining the important reactor parameters and state variables for adequate control requires an understanding not only of the plasma behavior itself but also of other influences on the plasma such as actions from the external coils and eddy currents induced in the first wall. An iterative process reducing the state variables with low sensitivities to input or output variables will be required to arrive at a reasonable set of state equations.

We define the following function spaces that apply to the fusion reactor system. The total space of state varlables is designated by $\Sigma$. The actual number of state variables used will be a subset of $\Sigma$ but may vary from the startup to the burn to the shutdown phase because the physics is different in each phase. In all cases the number will probably include $T_{i}, T_{e}, n_{2}, n_{3}$, and $n_{5}$. We designate the space of output variables or observables by $Y$ and look for the form of $\underline{y}$ that includes as few of these state variables as possible in order to lock in 
the desired operating condition of the reactor. Finally, the space of all possible input parameters is designated $U$, which includes deuterium and tritium input rates and energies, $\mathrm{OH}$ coil power supply voltage, EF power supply voltage, neutral beam injector heating, rf heating, and perhaps divertor coil voltage supply and impurity injection.

Control for startup and shutdown will, of necessity, be different from control for the burn phase because there are no nominal operating or steady-state parameters. The analogy to starting a fission reactor is somewhat applicable here in that many of the control inputs will be ramped in time from initial to burn values. Various startup scenarios have been simulated, ${ }^{3}$ but no serious considerations of controller hardware design have been attempted.for the combined position, shape, and stability problem. Controller hardware design is difficult because plasma diagnostic systems are in an early state of development. Further underștanding of plasma. mechanisms will accelerate diagnostic development and improve the control sensors necessary to a control concept formulation.

Once a plasma is formed, plasma position and shape, as well as the $q(x)$ profile, become matters of concern. It is likely that a time evolution of these parameters may be worked out a priori or by exper1ence. The parameters would be preprogrammed into the control system so that instead of an operating value $R_{0}$, a desired time-evolving $R_{0}$ would be used. In all cases it appears that startup state equations w111 be required and that an experimental or operational method for determining when ignition has been achieved must be developed. It is important to identify variables that describe the plasma behavior which can be sensed directly so that the computational time between the observation and the perturbation can be maximized to allow for control.

\section{A.3 PLASMA STARTUP}

The startup state equations would connect $\underline{u}_{s} \in U$ with the startup state reactor $\underline{x}_{s} \varepsilon \Sigma$ :

$$
\dot{\mathrm{x}}_{\mathrm{s}}=\mathrm{A} \underline{\mathrm{x}}_{\mathrm{s}}+\underline{\mathrm{B}}_{\mathrm{s}} \underline{\mathrm{u}}_{\mathrm{s}},
$$


where $A$ and $\underline{\underline{B}}$ are matrices defined by the physics of the reactor during the startup phase. ${ }^{14}$ The output variables are some function of the state variable so that $\underline{y}_{s} \varepsilon Y$ is related to $\underline{x}_{s} \varepsilon \Sigma$ :

$\underline{y}_{s}=\underline{c}_{s} \underline{x}_{s}$

We assume that $\stackrel{A}{=}_{S}, \stackrel{B}{=}_{S}$, and $\underline{\underline{C}}_{S}$ may be time varying but that they are not a function of any components of the state reactor $\underline{x}_{s}$.

The performance index for the startup phase is composed of both plasma position (vertical and horizontal) and stability-characterizing parameters. Perhaps the plasma position and shape could be adequately specified by the major plasma radius $R$ and the radial moments of the density distribution (or perhaps pressure distribution). by $\left\langle\mathrm{r}^{\mathrm{k}}\right\rangle$, where $k$ is less than or equal to some arbitrary number. This means that $R$ is the distance from the centerline of the torus to the point in the plasma where the temperature is at a maximum (assuming the plasma has no heavy ions) and that

$$
\left\langle\mathrm{r} k=\frac{\int_{0}^{2 \pi} \int_{0}^{1 \text { imiter }} \mathrm{r}_{\mathrm{k}(\mathrm{r}, \theta) \mathrm{r} \mathrm{dr} \mathrm{d} \theta}}{\int_{0}^{2 \pi} \int_{0}^{1 \text { imiter }} \mathrm{n}(\mathrm{r}, \theta) \mathrm{r} \mathrm{dr} \mathrm{d} \theta}, \mathrm{k}=1,2, \ldots . \mathrm{k} .\right.
$$

The $q(r)$ profile is derived from other measured parameters and may be used to characterize the stability of the plasma. Therefore, a suitable control scheme may be one in which the inputs $\underline{u}_{s}$ influence $\underline{x}_{s}$ so as to minimize the performance index:

$J_{s}=\int_{0}^{\infty}\left(w_{1} \tilde{R}^{2}+\sum_{k=1}^{k} w_{2}<\tilde{r}^{k}>^{2}+\sum_{\ell=1}^{L} w_{31} \tilde{q}_{e}^{2}+w_{4} \tilde{z}^{2}\right) d t$.

where $q_{e}$ is the safety profile at resonance surfaces and $L$ is the largest one that need be considered. The w's are weighting factors, and the tilde represents the difference between the actual value and the desired value of the parameter at time $t$. The effect of this quadratic 
performance index is to minimize deviations of the measured values of the state variables from the prescribed values obtained by calculations or experience.

A fundamental question is whether a decomposition of these spaces is possible in any of the phases. For example, if we look at the startup phase and choose input vector $\underline{u}_{s_{l}} \varepsilon U$ to control the shape and position of the plasma and $\underline{u}_{s_{2}}$ to control the stability, are the components of $\underline{u}_{s_{1}}$ all different from those of $\underline{u}_{s_{2}}$ ? Mathematically we desire

$\underline{\dot{x}}_{s}=\underline{\Delta}_{s} \underline{x}_{8}+\underline{B}_{s_{1}} \underline{u}_{s_{1}}$,

with

$\underline{y}_{s_{1}}=\underline{C}_{s_{1}} \underline{x}_{s}$

and

$\underline{\dot{x}}_{\mathrm{s}}=\stackrel{\mathrm{A}}{\mathrm{s}}_{\mathrm{s}} \underline{\mathrm{x}}_{\mathrm{s}}+\underline{\mathrm{B}}_{\mathrm{s}_{2}} \underline{\mathrm{u}}_{\mathrm{s}_{2}}$,

where

$\underline{\mathrm{y}}_{\mathrm{s}_{2}}=\underline{\mathrm{c}}_{\mathrm{s}_{2}} \underline{\mathrm{x}}_{\mathrm{s}}$

such that $\underline{u}_{s}$ is orthogonal to $\underline{u}_{s_{2}}$ and $\underline{y}_{s_{1}}$ is orthogonal to $\underline{y}_{s_{2}}$. If significant time delays occur in the state equations, it seems unlikely that such a separation will be possible.

The startup stage is characterizud by ohmlc heating, neutral beain injection, and then a transition into the ignition regime. This stage will take $210-20 \mathrm{~s}$ in the ETF, and then the burn phase of $2500 \mathrm{~s}$ will occur. The state variables in this stage are not yet well defined. Impurity concentrations may be critical because large radiation losses result if these become large for a given filling pressure. The energy losses due to chargè exchange, impurity line radiation, etc., may require 
additional heat input such as electron cyclotron resonance heating. As noted in Table A.2 the state equations and the instrumentation used in the startup phase will require concentration on the stability of the plasma.

\section{A. 4 PLASMA BURN PHASE}

Once the plasma is ignited, the control will probably have to be shifted to a different control concept that also accounts for the power level during the burn phase. It is possible that the power level need not be kept constant because it is the total energy over the burn cycle that 1 s to be maximized, 15 consistent with the demands of position, shape, and stability constraints. For the plasma to remain ignited, the thermal energy multiplication factor ${ }^{16} \mathrm{k}$ en must remain unity just as the neutron multiplication factor in a fission reactor must be one. However, there may be a fundamental difference between the fission neutron multiplication factor and the fusion thermal energy multiplication factor. The neutron multiplication factor can be unity at any power level at which the reactor is stable. On the other hand, the stability of the operating point of the fusion reactor when $\mathrm{k}_{\mathrm{en}}$ is unity is questionable at this time; thus, the control system may have to be better than that used in fission reactors.

An appropriate performance index for the burn phase of the cycle might be the particular control strategy that changes the burn phase reactor state variable $x_{-b} \varepsilon \sum$ so that

$J_{b}=\int_{0}^{T_{b}}\left(w_{4} \tilde{k}_{e n}^{2}+w_{5} \tilde{R}^{2}+\sum_{k=1}^{k} w_{6}<\tilde{r}>^{2}+\sum_{\ell=1}^{L} w_{7 \ell} \tilde{q}_{\ell}^{2}+w_{8} \tilde{z}^{2}\right)$

is minimized. Various constraints would likewise have to be imposed (e.g., if the wall loading exceeded a certain rate, shutdown would be initiated). T.f $\tilde{k}_{\text {en }}$ deviates much from zero, the power level of the reactor may shift drastically; thus, the weighting factor $w_{4}$ may need to be chosen carefully.

Even though there are nominal operating values for ion temperature, density, and electron temperature during the burn phase, they will 
probably change. Synchrotron radiation increases rapidly with electron temperature and with impurities caused by helium ash accumulation and by particles sputtering from the wall that change the characteristics of the plasma. The diffusion rate of tritium and deuterium may be different; therefore, an additional diagnostic that measures $n_{2}$ and $n_{3}$ separately may be required.

To suppress MPD's, control of the plasma $q(r)$ profile by neutral gas injection and plasma current control ${ }^{11}$ has been tried. Often a precursor to a MPD is the presence of other oscillations (sawtooth poloidal mode number $=1$; minor oscillation - poloidal mode number $=2$, $3, \ldots)$. Sawtooth oscillations abruptly change the electron temperature profile to a nonequilibrium state; thus, the shape control mechanism must not act in such a way as to force the plasma into an unstable configuration. The stability control will probably involve very complicated state equations so that on-line computation capability and realtime considerations may require drastic approximations unless data feedthrough problems and computational algorithms can be further analyzed to determine the relationship between computational time and the accuracy of the result. Of course, correlations to anticipated response times need comparisons, and DEMO-sized reactors may help in this control area.

\section{A. 5 SHUTDOWN PHASE}

of the three phases briefly discussed here, the shutdown phase has been studied the least. Shutdown may take $10 \mathrm{~s}$ or so and in some respects is a reversal of the process used to initiate the plasma, The time for shutdown to occur should be greater than the energy confinement time of the plasma so that heat load problems on the wall can be circumvented.

In the shutdown phase we again need to be concerned with plasma position and stability. Even during shutdown we want to avoid the occurrence of a MPD because of the potentially severe consequences to the wall. A performance index similar to Eq. (A.4) could be written except that now the state variables $\underline{x} \in \Sigma$ and the control variables $\underline{u}_{d}$ $\varepsilon U$ are different than they were in the startup phase. 
Shutdown control has not yet been studied in any detail. The discharge must'be terminated in a short time so that the burn time can be maximized to the cycle time. The shutdown time must be long enough so that the $200 \mathrm{MJ}$ of thermal energy stored in the plasma is not deposited on the wall so quickly that wall damage is done or that wall lifetime is significantly shortened.

It may be necessary to add heat during the shutdown phase to avoid an abrupt quench of the plasma that may result from impurity buildup, which could take the plasma to a subignition regime. All of the inputs available for control in the other two phases are potentially applicable here. Again, the fundamental difficulty is the lack of adequate state equations.

Some techniques suggested for shutdown include reducing the fuel input, injecting impurities, and enhancing alpha particle transport by means of a ripple coil. Understandably, the major effort to date has been on obtaining an ignited plasma. However, the imminence of the demonstration of fusion scientific feasibility supports the need for an increase in investigation of controlled termination.

\section{A.6 DIAGNOSTICS CONSIDERATIONS}

Control without diagnostics is impossible. The diagnostics must be very rapid compared to the time constants of the physical phenomena being controlled, i.e., plasma position, stability, and power. Additionally, the diagnostics must be able to withstand the environment of the ETF and be remotely maintained and calibrated. Table A.3 is a matrix of some detectors and some environmental conditions. A " $Y$ " means that the diagnostic can stand up in that environment, a question mark means either that we do not know or that it depends upon the exact nature of the detector, and an " $N$ " means that the diagnostic should not be used in that environment.

A list of plasma diagnostics is provided by Edelbaum et a1.17 The sensors will have to be calibrated remotely because neutron production will prevent manual calibration. 
Tabie A.3. The environmental impact on various diagnostic methods

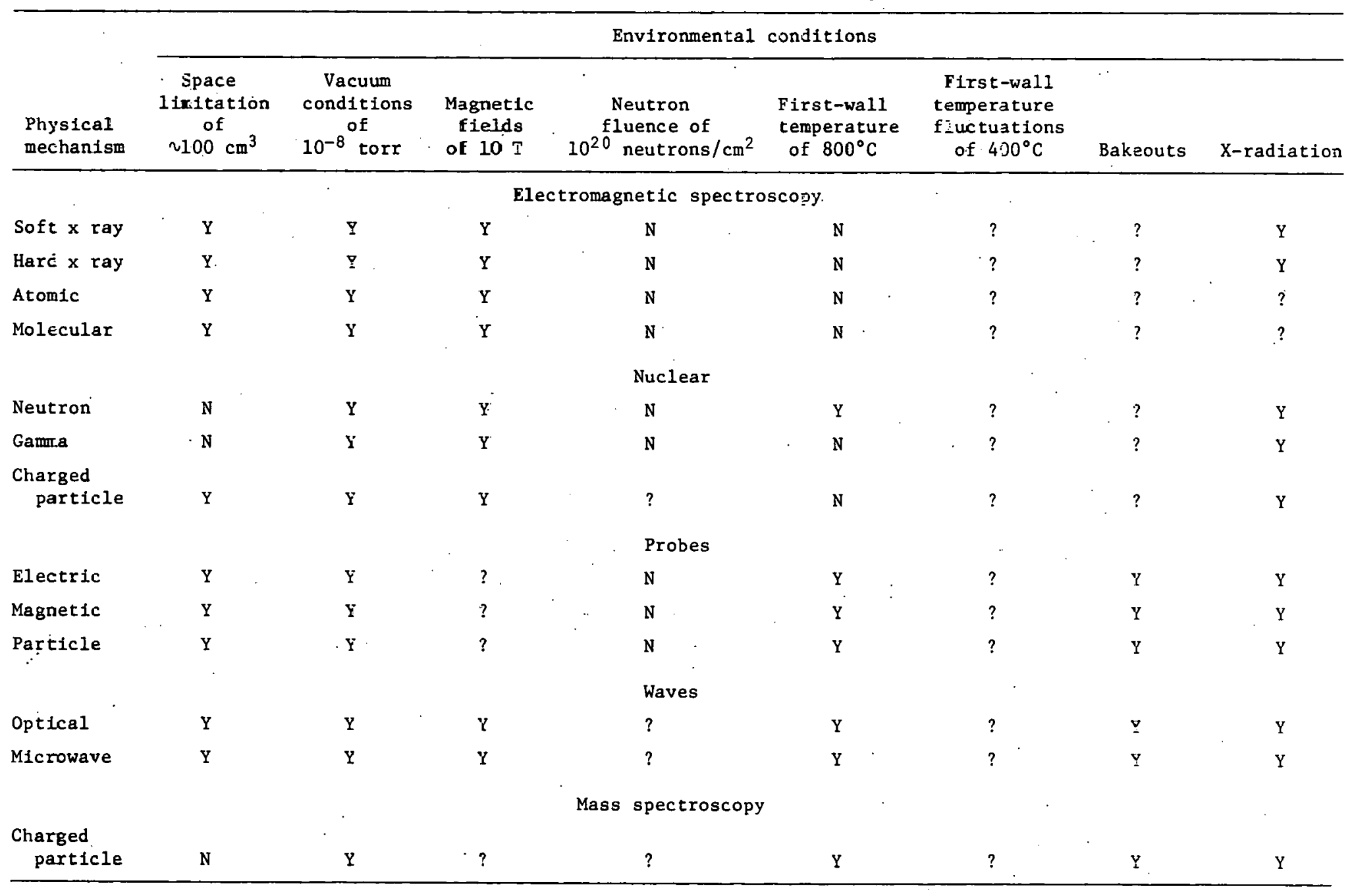


A significant factor that must be taken into account in future fusion systems is the high neutron fluxes in which the detectors must operate. The neutron loading for the ETF is expected to be $\sim 2.4 \times$ $10^{6} \mathrm{~W} / \mathrm{m}^{2}$ or $1.06 \times 10^{14}$ neutrons $/ \mathrm{cm}^{2} \cdot \mathrm{s}$ impinging on the first wall. This translates into a neutron fluence of $3.35 \times 10^{21}$ neutrons $/ \mathrm{cm}^{2}$ in one year of operation at maximum power. Most of the diagnostics will be located behind ports in the first wall, but neutrons will still have an influence in at least two ways.

(1) Neutrons are likely to make viewing ports (glass, beryllium, etc.) less transparent to laser beams, soft $x$ rays, ultrasoft $\mathrm{x}$ rays, etc.

(2) The transmutation of elements will probably cause the absorption of radiation to have a different frequency dependence than the port initially had.

The calibration of the detection instruments at intervals by remote means will be necessary for at least some of the diagnostics.

Computer software that takes and interprets the sensor data and obtains the parameters of interest for control must also be developed. Vast amounts of data from soft $x$-ray detector banks. will have to be analyzed in order to obtain relevant plasma parameters such as electron temperatures, shape, etc. Naturally, the control requirements are radically different from the experimental needs, and the amount of data can be held to a manageable level by using data collection bursts or windows.

For control purposes it would be desirable to be able to determine directly the ion temperature as a function of position because the fusion energy production rate is a sensitive function of temperature.

It may be possible to use synchrotron radiation as a control knob and as a diagnostic tool. Unfortunately, at the present time it is not very well analyzed. If a significant amount of energy is reflected back from the first wall, a port or variable opening in the wall may provide a means of fine-tuning the central temperature of the plasma. 
The detection of the plasma position and shape can probably be done very rapidly by means of soft $x$-ray silicon surface barrier detectors. The plasma emits soft $x$ rays consisting of bremsstrahlung, recombination, and line radiation. It seems that the intensity of the $x$ ray is thus proportional to the electron temperature $\mathrm{T}_{e}$. Ultrasoft $\mathrm{x}$ rays should be relatively insensitive to the electron temperature because ultrasoft $x$-ray transitions can be induced by thermal electrons. U1trasoft $x$ rays are primarily the result of impurities and so should be proportional to the startup phase density $\mathrm{n}_{\mathrm{s}^{\cdot}}$. Plasma disruptions have been studied on very short time scales by this techmique. 18 These preliminary diagnostics considerations underscore the fact that any contro] roncept will require additional observations on the plasma that will provide data to generate the control knob intelligence.

\section{A. 7 SUMMARY}

Control of fusion devices is not presently developed to any degree except for position control. The basic difficulty is that simple state equations suitable for control are not known. Even though the ETF is still about a decade away, serious attention to the developments needed for its control is essential.

Because of the nonlinear state equations modern control theory 3ccms to pruvide the most appropriate forum for discussing fusion control. Serious considerations about time constants and the separability of position control from power and stability control may permit easier control than is currently anticipated.

Diagnostles suitable for ETF control are also inadequately developed. A great deal of computer software and data-handling techniques sultable for plasma control is also necessary. The performance indicca described above require the development of nuitable lardwarc, computer sullware, and control algorithms. However, the performance indices chosen are a reflection of the current understanding of the plasma physics involved and may need to be altered or completely changed.

Even though some work has been done in the past on stability control, no currently operating devices have incorporated stability 
control to any extent. Sen indicates that it is theoretically possible to use one properly placed sensor and a single suppressor (control knob) to stabilize all unstable modes. ${ }^{19^{\circ}}$ Much work is still required in all three areas of fusion plasma control.

Finally, it has been noted that analytically it is possible to design an automatic control system that can selectively detect and stabilize some of the helical flux modes of a plasma column. ${ }^{20}$ Most of the work done on stability thus far has been done in cylindrical rather than toroidal geometry. It may be that this will not cause many difficulties, but the toroidicity of the plasma certainly has some effect on particle transport. Toroidal geometry may accentuate instabilities so that faster dynamic response is necessary. A proper set of state equations, including confinement times and a particle transport representation that reflects the physics and the toroidal geometry, requires development.

\section{REFERENCES}

1. D. J. Rose, Nuc1. Fusion 9, 183-230 (1969).

2. D. Steiner et al., ORNL/TM-6201, Oak Ridge, Tennessee (1978).

3. W. R. Becraft et al., ORNL/TM-6617, Oak Ridge, Tennessee (1979).

4. T. K. Chu and H. W. Handel, eds., Feedback and Dynamic Control of PZasmas, American Institute of Physics, New York, 1970.

5. J. A. Holmes et al,, ORNL/TM-6707, Oak Ridge, Tennessee (1979).

6. R. Gran, M. J. Rossi, and F. Sobierajski, Proc. 7th Symp. on Engineering Problems of Fusion Kesearch, pp. 104-111 (1978).

7. A. Ogata and H. Ninomiya, Jpn. J. Appl. Phys. 18, 825-834 (1979).

8. D. E. Voss, K. Bol, and J. L. Johnson, PPPL-1483, Princeton, New Jersey (1978).

9. W. W. Saylor, "Plasma Position Control in a Tokamak Experimental Power Reactor," M.S. Thesis, Massachusetts Institute of Technology, Cambridge, Massachusetts, 1977.

10. J. L. Anderson et a1., Nuc1. Fusion 16, 629-637 (1976). 
11. J. Fujita et al., "Suppression of Major Disruption by Neutral. Gas Injection and Plasma Current Control in J.I.P.P. I-II Torus," IAEA-CN-37-N-2, pp. 1-9, Institute of Plasma Physics, Nagoya University, Nagoya, Japan, 1979.

12. S. Tamor, Nuc1. Fusion 18, 229-241 (1978).

13. A. L. Greensite, Elements of Modem Control Theory, Vo1. I, Spartan Books, New York, 1970.

14. A. T. Mense et al., Proc. 3rd Topical Meeting on the Technology of Controlzed Nuclear Eusion, Vol. II, pp. 833-842 (1978).

15. P. A. Martini and R. J. Onega, Nuc1. Technol. 36, 285-293 (1977).

16. J. R. MuNally, Jr., Nucl. Fusion 17, 1273 (197\%).

17. T. N. Edelbaum, P. A. Madden, and R. E. Var, EPRI ER-533, Palo Alto, California (1977).

18. D. R. Eames et al., PPPL-1530, Princeton, New Jersey (1979).

19. A. K. Sen, IEEE Trans. Flasma Sci., Vol. PS-7, pp. 116-119 (1979).

20. R. A. D'emirkhanov et al., Sov. J. Plasma Phys. 1, 392-395 (1975). 


\section{APPENDIX B}

THE EXPERIMENTAL DETERMINATION OF THE DEUTERON-TRITON RATIO IN A FUSION PLASMA

Two pellet injectors, one for injecting deuterons and the other for injecting tritons, will probably be used in the control of a fusion reactor. Although the power output of the device depends upon the relative number of deuterons and tritons, this output is not in itself a sensitive measure of the deuteron and trition densities even if the ion temperature is known. Control using both pellet injectors requires diagnostics that will determine the relative average $D-T$ densities at time $t$. Keeping a 50-50 ratio of deuterons and tritons maximizes the power output for a given temperature and a given number of particles. However, it may be that high-temperature operation (35-75 keV) may be advantageous because of different diffusion mechanisms now coming into prominence.

Letting $\langle\sigma v\rangle_{22}$ and $\langle\sigma v\rangle_{23}$, denote the neutron-producing $D-D$ and $D-T$ reaction rates and assuming equal rates for the two branches of the: D-D reaction, the particle balance equations for deuterons and tritons respectively are

$$
\frac{d n_{2}(t)}{d t}=S_{2}-2\langle u v\rangle_{22} n_{2}^{2}-\langle\sigma v\rangle_{23} n_{2} n_{3}-\frac{n_{2}}{T_{2}}
$$

and

$$
\frac{\mathrm{dn}_{3}(t)}{\mathrm{dt}}=\mathrm{s}_{3}+\langle\sigma v\rangle_{22} \mathrm{n}_{2}^{2} / 2-\langle\sigma v\rangle_{23} \mathrm{n}_{2} \mathrm{n}_{3}-\frac{\mathrm{n}_{3}}{\mathrm{~T}_{3}} .
$$

In principle the $D-T$ ratio can be measured by taking either the ratio of terms within an equation or the ratio of terms from each equation. Therefore, there are two options.

(1) One can determine the neutron production terms by taking the ratio of two terms in the same equation or the ratio of the neutron production terms in each reaction: 
$\frac{N_{2}}{N_{3}}=\frac{\langle\sigma v\rangle_{22} n_{2}^{2} / 2}{\langle\sigma v\rangle_{23} n_{2} n_{3}}=\frac{n_{2}(t)}{n_{3}(t)} \frac{\langle\sigma v\rangle_{22} / 2}{\langle\sigma v\rangle_{23}} ;$

the evaluation of $\langle\sigma v\rangle_{22} /\langle\sigma v\rangle_{23}$ requires a knowledge of the average ion temperature.

(2) One can use a mass spectrographic method to measure $\left(\mathrm{n}_{2} / \mathrm{T}_{2}\right) /\left(\mathrm{n}_{3} / \mathrm{T}_{3}\right)$ in the collection chamber of the divertor and then relate this back to the density in the plasma.

We will look first at lle weutron detection method.

The two itacliuns of interest that produce neutrone arc $T+D$, $3.52 \mathrm{MeV}+\mathrm{n}(14.07 \mathrm{MeV})$ and $\mathrm{D}+\mathrm{D} \rightarrow{ }^{3} \mathrm{He}(0.82 \mathrm{MeV})+\mathrm{n}(2.425 \mathrm{MeV})$. The number of neutrons prontused per $\mathrm{cm}^{3} \cdot \mathrm{f}$ from the $\mathrm{D}-\mathrm{T}$ reacliun is

$N_{23}(\vec{r}, t)=n_{2}(\vec{r}, t) n_{3}(\vec{r}, t)<\sigma v>_{23}(\vec{r}, t)$,

and the $D-D$ neutron production rate is

$\left.N_{22}(\vec{r}, t)=n_{2}^{2}(\vec{r}, t)<\sigma v\right\rangle_{22} / 2$

The neutron production rate will vary throughnut the plasma volumc. $\Lambda$ ssuming a cylindrical volume for the plasma that produces the neutrons seen by the detector, we can relate the production rate to the densities as follows. As seen in Fig. B.1, assuming there is no density or temperature variation over the cross-sectional area of the cylinder, the total number of neutrons produced in the $\mathrm{D}-\mathrm{T}$ reaction along the diametral chord is

$N_{23}(t)=2\left[\int_{0}^{a} n_{2}(r, t) n_{3}(r, t)<\sigma v>232 \pi r d r\right] \pi \frac{d^{2}}{4}$

The neutrons seen by the detector in the first wall are reduced by a solid angle factor $\Omega_{3}$; therefore,

$N_{23}(t)=N_{23}^{\prime}(t) \Omega_{3}=\Omega_{3} n_{2}(t) n_{3}(t)\langle\sigma v\rangle_{23}$. 


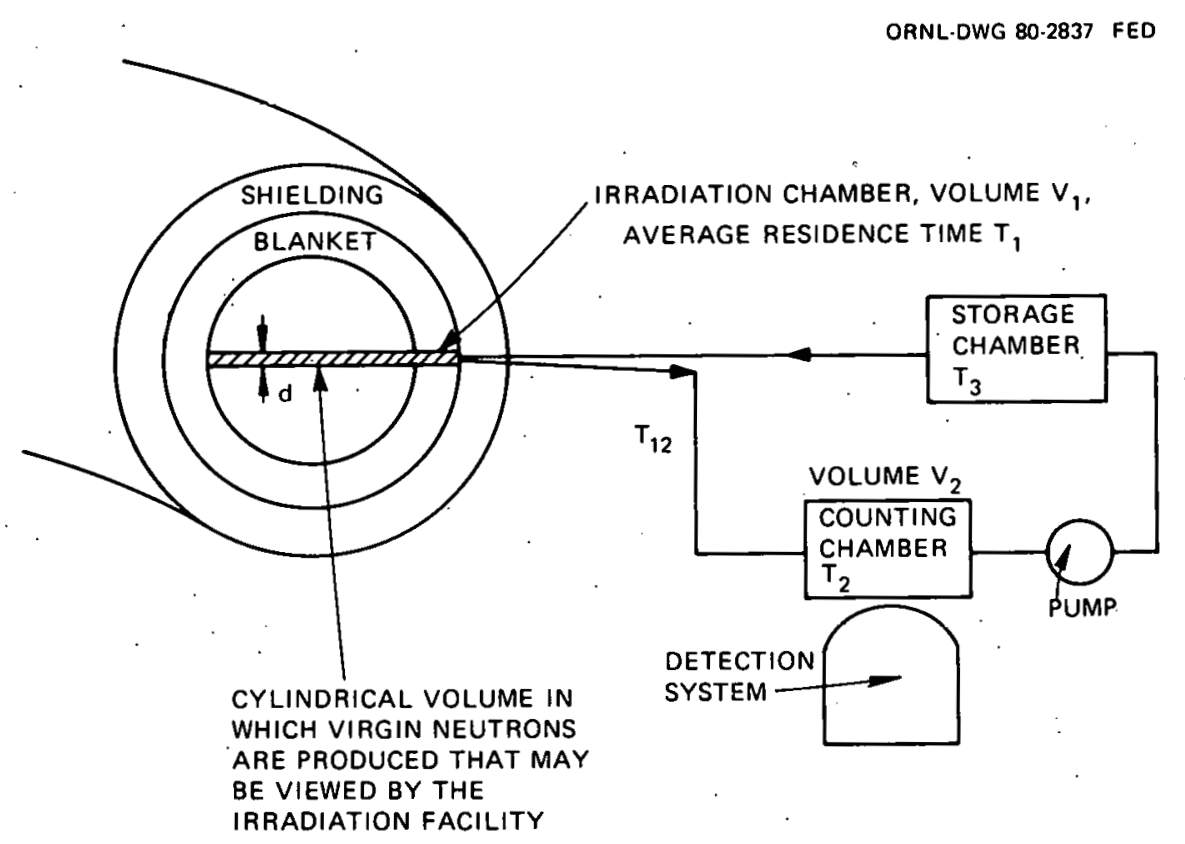

Fig. B.1. Schematic diagram of the neutron detection system for the experimental determination of the deuteron and triton density. 
Similarly, the number of $D-D$ neutrons striking the detector is

$N_{22}(t)=\Omega_{2} n_{2}^{2}(t)\langle\sigma v\rangle_{22} / 2$

The ratio of neutrons striking the detector is thus $\left(\Omega_{2}=\Omega_{3}\right)$

$\frac{N_{22}(t)}{N_{23}(t)}=\frac{n_{2}(t)\langle\sigma v\rangle_{22}(t)}{2 n_{3}(t)\langle\sigma v\rangle_{23}(t)}$.

Of course, $\langle\sigma v\rangle_{22}$ in F.n. (B.9) 1c for the neullull-producing branch of the $\mathrm{D}-\mathrm{D}$ reaction only.

The detection system for determining $\|_{2}(t) / n_{3}(t)$ must have the following characteristics for fusion reactors.

(1) It must be able to operate in a high-temperature area $\left(300-500^{\circ} \mathrm{C}\right)$.

(2) Because both $x$ rays and scattered neutrons will impinge on the detector, it must be able to discriminate against such radiation background.

(3) Because the reactor will likely be cycled, it must be able to tolerate thermal cycling.

(4) The shorter the response time of the detector Llie betcer. Kesponse timcs of milliseconds or less are desirable.

(5) Some neutron discrimination is necessary to sort the 14.1- and 2.4-MeV neutrons from each other. These energies are not as sharp as indicaled here. 1

A threshold fission chamber would satisfy all of these characteristics except that the threshold energies arc incorrect and the fission cross sections are much too small (for example, for the fission of bismulh). No fissiondule 1sotopes are available that have all of the characteristics necessary to produce a reasonable detector.

The following method is considered for determining the relative densities of deuteron and triton. Isotopes that undergo threshold reactions are dissolved in water or some other fluid (e.g., carbon tetrachloride) and pumped continuously into the reactor and back out to 
a counting chamber, as shown in Fig. B.1. Table B.1 lists some data on threshold reactions that may be useful in the detection system. Because of the scattering of high-energy neutrons, a continuous neutron spectrum is likely. At least three isotopes that undergo threshold reactions at roughly $13.0 \mathrm{MeV}, 3.0 \mathrm{MeV}$, and $2.0 \mathrm{MeV}$ are chosen to bracket the neutrons to be detected. The count rate from the $13.0-\mathrm{MeV}$ threshold detector will be sensitive only to the $14.1-\mathrm{MeV}$ virgin neutrons, and the difference in the count rate of the $3.0-$ and $2.0-\mathrm{MeV}$ threshold reactions, corrected for cross sections, half-lives, etc., should provide the 2.425-MeV neutron intensity.

This system would have at least the following operational advantages.

(1) The irradiation chamber could be placed next to the first wall because the heat could be taken away by the fluid flow.

(2) The fluid could be pumped into a near-radiation-free area; therefore, the counting system would not need to be subjected to such high neutron fields.

(3) The half-lives, threshold energies, counting systems, irradiation and storage volumes, and pumping rate would all be, up to a point, a matter of choice for the diagnostician.

The primary disadvantage is that in the reactor the counting rate information lags significantly behind the neutron production rate. The lag time can be of the order of a second or less.

In order to evaluate the feasibility of this flow-counting system, we define the following symbols:

$$
\begin{aligned}
\mathrm{n}_{2}(\overrightarrow{\mathrm{r}}, t)= & \text { the deuteron density at position } \overrightarrow{\mathrm{r}} \text { at time } t \text { in the plasma, } \\
\mathrm{n}_{3}(\overrightarrow{\mathrm{r}}, \mathrm{t})= & \text { the triton density at position } \overrightarrow{\mathrm{r}} \text { at time } t \text { in the plasma, } \\
\mathrm{N}_{3 i}= & \text { the number of atoms } / \mathrm{m}^{3} \text { of the fluid that has a threshold for } \\
& \text { deterting the } 14.06-\mathrm{MeV} \text { neutrons from the } \mathrm{D}-\mathrm{T} \text { reaction, } \\
\mathrm{N}_{3 \mathrm{f}}= & \text { the number of product atoms } / \mathrm{m}^{3} \text { of fluid as a result of the } \\
& 14-\mathrm{MeV} \text { neutron reaction, } \\
\mathrm{N}_{2 i}= & \text { the number of atoms/m of the fluid that has a threshold for } \\
& \text { detecting the } 2.5-\mathrm{MeV} \text { neutrons, }
\end{aligned}
$$


Table B.1. Isotopes that may be useful in determining the $\mathrm{n}_{2}(t) / \mathrm{n}_{3}(t)$ ratio in a fusion reactor

\begin{tabular}{|c|c|c|c|c|c|}
\hline Isotope & Reaction & $\begin{array}{c}\text { Threshold } \\
\text { energy } \\
(\mathrm{MeV})\end{array}$ & $\begin{array}{l}\text { Product } \\
\text { half-life } \\
\text { (s) }\end{array}$ & $\begin{array}{l}\text { Cross } \\
\text { section } \\
(\mathrm{mb})^{a}\end{array}$ & Compound \\
\hline $\mathrm{C} 1-37$ & ${ }^{37} \mathrm{C}(\mathrm{n}, \alpha){ }^{34} \mathrm{P}$ & 1.4 & $12.4(\beta, \alpha)$ & 44 & $\mathrm{CCl}_{4}$ \\
\hline$B-11$ & ${ }^{11}{ }_{B}(n, \alpha){ }^{8} \mathrm{Li}$ & 6.63 & $0.844\left(B^{-}\right)$ & 33 & Boric acid \\
\hline $0-16$ & ${ }^{16} \mathrm{O}(\mathrm{n}, \mathrm{P})^{16} \mathrm{~N}$ & 9.62 & $7.10\left(\beta^{-}, \gamma\right)$ & 40 & $\mathrm{H}_{2} \mathrm{O}$ \\
\hline $\mathrm{C}-12$ & ${ }^{12} \mathrm{C}(\mathrm{n}, \mathrm{P}){ }^{12} \mathrm{~B}$ & 12.6 & $0.0204(\beta, \gamma)$ & $?$ & $\mathrm{CCl}_{4}$ \\
\hline $\mathrm{Na}-2.3$ & $23^{3} \mathrm{Na}(\mathrm{n}, \mathrm{P})^{23^{3} \mathrm{Ne}}$ & 3.6 & $37.5(\beta, \gamma)$ & $\backsim 5$ & $\mathrm{NaCl}$ \\
\hline$\dot{A} \cdot 1=27$ & ${ }^{27} \Lambda 1(n, 2 n)^{26} \mathrm{Al}$ & 13.05 & $6.34\left(B^{+}\right)$ & $\tau(1) .1$ & $\mathrm{Al}_{2} \mathrm{O}_{3}$ \\
\hline
\end{tabular}


$\mathrm{N}_{2 \mathrm{f}}=$ the number of product atoms $/ \mathrm{m}^{3}$ as a result of the reaction,

$N_{1 i}=$ the number of atoms $/ \mathrm{m}^{3}$ of the fluid that has a threshold for detecting the 2.3-MeV neutrons,

$\lambda_{3}, \lambda_{2}, \lambda_{1}=$ the decay constants for $N_{3 f}, N_{2 f}$, and $N_{1 f}$, respectively $\left(s^{-1}\right)$,

$\mathrm{T}_{1}=$ the average atom residence time in the irradiation chamber,

$\mathrm{T}_{2}=$ the average atom residence time in the counting container,

$\mathrm{T}_{3}=$ the average atom residence time in the storage chamber,

$\sigma_{3}=$ the cross section for the $14.1-\mathrm{MeV}$ threshold reaction,

$\sigma_{2}=$ the cross section for the $3.0-\mathrm{MeV}$ threshold reaction,

$\sigma_{1}=$ the cross section for the $2.0-\mathrm{MeV}$ threshold reaction,

$\phi_{3}=$ the $14.1-\mathrm{MeV}$ neutron flux at the irradiation facility, and

$\mathrm{T}_{12}=$ the average transit time from the irradiation chamber to the detection chamber.

If the atoms $N_{3 i}$ are irradiated at a time $t$, then the number of radioactive product atoms $\mathrm{N}_{3 f}$ that reach the detection chamber a time $\left(\mathrm{T}_{1} / 2\right)+\mathrm{T}_{12}$ later is

$N_{3 f}\left[t+\left(\frac{T_{1}}{2}\right)+T_{12}\right]=N_{3 f}(t) e^{-\lambda_{3}\left[\left(T_{1} / 2\right)+T_{12}\right]}$.

The number of atoms at time $t$ in the irradiation chamber is

$N_{3 E}(t)=\frac{N_{3 i} \sigma_{3} \phi}{\lambda_{3}}\left[1-e^{-\lambda_{3}\left(T_{1} / 2\right)}\right]$

The average time that an atom spends in the counting container is $\mathrm{T}_{2}$; thus, it spends roughly $\mathrm{T}_{2} / 2 \mathrm{~s}$ in this chamber before it decays (actually an exponentially weighted average should be taken). Therefore, 


$$
\begin{aligned}
\mathrm{N}_{3 \mathrm{f}}\left[t+\left(\frac{\mathrm{T}_{1}}{2}\right)\right. & \left.+\mathrm{T}_{12}+\left(\frac{\mathrm{T}_{2}}{2}\right)\right]=\mathrm{N}_{3 \mathrm{f}}(\mathrm{t}) \mathrm{e}^{-\lambda_{3}\left[\left(\mathrm{~T}_{1} / 2\right)+\mathrm{T}_{12}+\left(\mathrm{T}_{2} / 2\right)\right]} \\
& =\frac{\mathrm{N}_{3 i \sigma_{3} \phi}}{\lambda_{3}}\left[1-\mathrm{e}^{-\lambda_{3}\left(\mathrm{~T}_{1} / 2\right)}\right] \mathrm{e}^{-\lambda_{3}\left[\left(\mathrm{~T}_{1} / 2\right)+\mathrm{T}_{12}+\left(\mathrm{T}_{2} / 2\right)\right]} .
\end{aligned}
$$

The flux $\phi_{3}$ is related to the $\mathrm{D}-\mathrm{T}$ reaction rate as well as to the geometric configuration of the irradiation chamber on the wall. From Eq. (B.7), putting in a geometric factor for the fraction of the neutrons that can directly impinge on the irradiation chamber, we have

$$
\begin{aligned}
& N_{3 f}\left[t+\left(\frac{T_{1}}{2}\right)+T_{12}+\left(\frac{T_{2}}{2}\right)\right] . \\
&=\frac{N_{3 i} \sigma_{3}}{\lambda_{3}} \Omega_{3} n_{2}(t) n_{3}(t)\left\langle\sigma v>_{23} e^{-\lambda_{3}\left[\left(T_{1} / 2\right)+T_{12}+\left(T_{2} / 2\right)\right]}\right. \\
& \times\left[1-e^{-\lambda_{3}\left(T_{1} / 2\right)}\right] .
\end{aligned}
$$

The detection rate is some constant $\varepsilon_{3}$ (for detector efficiency and solid angle) and depends on the activity of $N_{3 F}(t)$. Setting $T$ equal to $\left(T_{1} / 2\right)+T_{12}+\left(T_{2} / 2\right)$, we hàve

$$
\begin{aligned}
A_{3}(t+T)=\lambda_{3} N_{3 f}(t+T) & \\
& =N_{3 i} \sigma_{3} \Omega_{3} \varepsilon_{3} n_{2}(t) n_{3}(t)<\sigma v>_{23} e^{-\lambda_{3} T}\left[1-e^{-\lambda_{3}\left(T_{1} / 2\right)}\right] .
\end{aligned}
$$

Similarly, for the detection of the neutrons from the D-D reaction, we have (if $\varepsilon_{1}=\varepsilon_{2}$ and $\Omega_{1}=\Omega_{3}$ ) 


$$
\begin{aligned}
A_{2}(t+T)=\lambda_{1} N_{1 f}(t+T) & -\lambda_{2} N_{2 \dot{f}}(t+T) \\
= & \varepsilon_{2}\left\{\phi_{1}(t) N_{1 i} \sigma_{1} e^{-\lambda_{1} T} \cdot\left[1-e^{-\lambda_{1}\left(T_{1} / 2\right)}\right]\right\} \\
& -\phi_{2}(t) N_{2 i} \sigma_{2} e^{-\lambda_{2} T}\left[1-e^{-\lambda_{2}\left(T_{1} / 2\right)}\right] .
\end{aligned}
$$

The fluxes $\phi_{1}$ and $\phi_{3}$ are related in that $\phi_{1}$ is due not only to. neutrons but also to the D-T neutrons; therefore,

$\phi_{1}(t)=\phi_{b}(t)+\Omega_{1} n_{2}^{2}\langle\sigma v\rangle_{22} / 2=\phi_{2}(t)+\Omega_{1} n_{2}^{2}\langle\sigma v\rangle_{22} / 2$.

Putting Eq. (B.16) into Eq: (B.15) we have

$$
\begin{aligned}
& A_{2}(t+T)=\varepsilon_{2}\left(\phi _ { 2 } ( t ) \left\{N_{1 i} \sigma_{1} e^{-\lambda_{1} T}\left[1-e^{-\lambda_{1}\left(T_{1} / 2\right)}\right]\right.\right. \\
& \left.-\mathrm{N}_{2 i} \sigma_{2} \mathrm{e}^{-\lambda_{2} \mathrm{~T}}\left[1-\mathrm{e}^{-\lambda_{2}\left(\mathrm{~T}_{1} / 2\right)}\right]\right\}+\Omega_{1} \mathrm{n}_{2}^{2} \frac{\langle\sigma \mathrm{v}\rangle_{22}}{2} \\
& \left.\times \mathrm{N}_{1_{i} \sigma_{1}} \mathrm{e}^{-\lambda_{1} \mathrm{~T}}\left[1-\mathrm{e}^{-\lambda_{1}\left(\mathrm{~T}_{1} / 2\right)}\right]\right) \text {. }
\end{aligned}
$$

Now we choose $\mathrm{N}_{l_{i}}$ and $\mathrm{N}_{2 i}$ (or the volumes if $\mathrm{N}_{1 i}$ and $\mathrm{N}_{2 i}$ are the same) so that

$N_{1 i} \sigma_{1} e^{-\lambda T}\left[1-e^{-\lambda_{1} \overline{\left(T_{1} / 2\right)}}\right]=N_{2 i} \sigma_{2} e^{-\lambda_{2} T}\left[1-e^{-\lambda_{2}\left(T_{2} / 2\right)}\right]$.

By eliminating these terms one can virtually eliminate this effect of the background.

Now we have the ratio of the D-D to D-T activity: 


$$
\begin{aligned}
& \frac{A_{2}(t+T)}{A_{3}(t+T)}=\frac{\varepsilon_{2} \Omega_{1} n_{2}^{2}(t)\left\langle\sigma v>_{22} N_{1 i} \sigma_{1} e^{-\lambda_{1} T}\left[1-e^{-\lambda_{1}\left(T_{1} / 2\right)}\right]\right.}{\left.2 \varepsilon_{3} \Omega_{3} n_{2}(t) n_{3}(t)<\sigma v\right\rangle_{23 N_{3 i} \sigma_{3}} e^{-\lambda_{3} T}\left[1-e^{-\lambda_{3}\left(T_{1} / 2\right)}\right]} \\
& =\frac{1 \mathrm{n}_{2}(\mathrm{t}) \sigma_{1}<\sigma v>_{22} \mathrm{~N}_{1 i} \mathrm{e}^{-\lambda_{1} \mathrm{~T}}\left[1-\mathrm{e}^{-\lambda_{1}\left(\mathrm{~T}_{1} / 2\right)}\right]}{2 \mathrm{n}_{3}(\mathrm{t}) \sigma_{3}\langle\sigma \mathrm{v}\rangle_{23} \mathrm{~N}_{3 i} \mathrm{e}^{-\lambda_{3} \mathrm{~T}}\left[1-\mathrm{e}^{-\lambda_{3}\left(\mathrm{~T}_{1} / 2\right)}\right]} .
\end{aligned}
$$

If we use an aluminum isotope, then the volume must be constructed so that the resident times are such that Eq. (B.18) is satisfied. The particular isotopes and reactions must be chosen carefully. In general, the $(n, 2 n)$ reaction has two advantages over other reactions.

(1) The products of the reaction, neutrons, do not need to overcome the Coulomb barrier and thus are not suppressed from leaving the nuclcus: The cross sections thereby rise very sharply to their maximum values.

(2) The threshold energy is generally very high ( $>5 \mathrm{MeV}$ ); therefore, it can be used to distinguish the $14-\mathrm{MeV}$ neutrons from all the lowerenergy neutrons.

The main difficulty is choosing isotopes that

(1) dissolve readily in water,

(2) have a half-life of a second or less, and

(3) have a high cross section for the $(n, 2 n),(n, \alpha)$, or $(n, P)$ reaction, depending on the reaction used.

As noted in Table B.1, 16.0 undergoes an (n, P) reaction and has a threshold of $9.26 \mathrm{MeV}$. It can be used al.ong with other isotopes to determine the $\mathrm{D}-\mathrm{T}$ reaction rate if the flow material is water. In addition, it has a very high-energy gamma ray $(6.3 \mathrm{MeV})$ from the ${ }^{16} \mathrm{~N}$ decay. Aluminum has all three'reactions with roughly the right threshold energies. However, ${ }^{27} \mathrm{Mg}$ and ${ }^{14} \mathrm{Na}$ are too long for use in fusion reactor control.

'l'herefore, from all considerations it seems that the proper compounds and isotopes for the control of a fusion reactor are those shown in Table B.1. These cross sections are all in the millibarn range. 
Using Eq. (B.1) and taking typical numbers we have $A_{2}(t+1) /$ $A_{3}(t+1)=10^{-2}$. To estimate the absolute correct rate that may be expected for $\left[A_{2}(t+T)\right]$, we use Eq. (B.17) (all cgs units):

$$
\begin{aligned}
\mathrm{A}_{2}(\mathrm{t}+\mathrm{T})= & \varepsilon_{2} \Omega_{1} \frac{\mathrm{n}_{2}^{2}}{2}\left\langle\sigma v{ }_{22} \mathrm{~N}_{11} \sigma_{1} \mathrm{e}^{-\lambda_{1} \mathrm{~T}}\left[1-\mathrm{e}^{-\lambda_{1}\left(\mathrm{~T}_{1} / 2\right)}\right]\right. \\
\cong & (0.1)\left(10^{-2}\right) \frac{\left(5 \times 10^{-13}\right)^{2}}{2}\left(5 \times 10^{-18}\right)\left(6 \times 10^{22}\right) \\
& \times\left(3 \times 10^{-27}\right) \mathrm{e}^{-1}\left(1-\mathrm{e}^{-0.2}\right)=75 \mathrm{Cts} / \mathrm{s} .
\end{aligned}
$$

An advantage of using the mass spectrographic method is that the time lag can be reduced to milliseconds, which is almost essential for feedback control. The disadvantage is that it is difficult to relate the material collected to the actual density in the plasma because the material has diffused into the scrape-off region, mixed with the deuterium and tritium components there, and then come to. the divertor plate.

The collector plates or the "lithium rain"2 will be located outside the TF coils. We assume the lithium droplet collector and note that the rate at which the ions enter the chamber must equal their capture rate by the lithium drops except for those that are uncollected and neutralized. The uncollected neutral background could be analyzed to determine the relative amounts in the plasma, hoping that $T_{2}$ and $T_{3}$ are at least proportional to each other.

\section{REFERENCES}

1. H. Liskien, Nuc1. Sci. Eng. 71, 57 (1979).

2. W. M. Wells, ORNL/TM-6727, Oak Ridge, Tennessee (1979). 


\section{THIS PAGE}

\section{WAS INTENTIONALLY \\ LEFT BLANK}




\section{APPENDIX C \\ THE FEASIBILITY OF USING SYNCHROTRON RADIATION \\ AS A FINE-TUNING TEMPERATURE CONTROL}

Here we present a preliminary examination of the possibility of using synchrotron radiation to control plasma temperature. The power terms of interest in evaluating the importance of synchrotron radiation ${ }^{1}$ are (1) the fusion power imparted to the plasma $\left(\mathrm{keV} / \mathrm{m}^{3} \cdot \mathrm{s}\right)$,

$\mathrm{P}_{\mathrm{F}}=\mathrm{n}_{2} \mathrm{n}_{3}\langle\sigma \mathrm{v}\rangle_{23}(3520) ;$

(2) the bremsstrahlung radiation $\left(\mathrm{keV} / \mathrm{m}^{3} \cdot \mathrm{s}\right)$,

$\mathrm{P}_{\mathrm{Br}}=\frac{3}{2} \mathrm{~K}_{3} \mathrm{n}_{\mathrm{e}} \mathrm{T}_{\mathrm{e}}^{1 / 2}\left(0.006 \mathrm{n}_{\mathrm{e}} \mathrm{T}_{\mathrm{e}}+\underset{i}{\Sigma} \mathrm{n}_{\mathrm{i}} \mathrm{Z}_{i}^{2}\right)$

and (3). the synchrotron (cyclotron) radiation $\left(\mathrm{keV} / \mathrm{m}^{3} \cdot \mathrm{s}\right)$,

$P_{c y}=\frac{3}{2} K_{2} n_{e} \frac{B^{5 / 2} T_{e}^{1 / 4}}{\sqrt{n_{e}}}(I-R)\left(1+\frac{T_{e}}{204}\right)$.

Here

$n_{2}, n_{3}, n_{i}, n_{e}=$ the average density of the deuterons, tritons, the ith ion specie, and electrons, respectively;

$\mathrm{T}_{e}, \mathrm{~T}_{i}=$ the average electron and ion temperatures, respectively;

$B=$ the internal magnetic field in the plasma;

$\mathrm{K}_{2}=10^{5} / \sqrt{\mathrm{L}}$, where $\mathrm{L}$ is the characteristic plasma length;

$\mathrm{K}_{3}=2 \times 10^{-21}$

$\mathbf{R}=$ the wall reflectivity for synchrotron radiation, which depends upon the wavelength of the emitted radiation; and

$z_{i}=$ the atomic number of the ith ion specie.

The bremsstrahlung radiation lies principally in the $x$-ray region. ${ }^{2}$ The synchrotron radiation emitted from a relativistic electron gas of uniform density $\mathrm{n}_{\mathrm{e}}$ and thickness $\mathrm{L}$. embedded in a constant uniform magnetic 
field $B_{0}$ exhibits a critical frequency $\omega^{*}$ so that if $\omega \leq \omega^{*}$, then the plasma behaves like a blackbody and if $\omega \geqslant \omega^{*}$, then the plasma is transparent to synchrotron radiation. The critical frequency $\omega *$ lies in the far-infrared range, where all metallic walls are good reflectors.

A dimensionless length $\mathrm{L}$ is often used in connection with synchrotron radiation. The dimensionless length is related to the actual plasma length, that is, the minor radius a, by the relation

$L=a \frac{\omega_{p e}^{2}}{C \omega_{0}}$,

where the plasma frequency is

$\omega_{p e}^{2}=\frac{n_{e} e^{2}}{m_{e} \varepsilon_{n}}$

and the cyclotron frequency is

$\omega_{v}=\frac{e B_{o}}{m_{e}}$.

Therefore, the dimensionless length that characterizes the plasma thickness to the emission of synchrotron radiation is

$L=\frac{a}{c} \frac{n_{e} e}{\varepsilon_{0} b}$.

The interilal magnetic field $B$ is related to the average beta in the plasma by

$\mathrm{R}=\sqrt{1-\beta} \mathrm{B}_{\mathrm{o}}$. 
Using a11 the relations above, with $\mathrm{R}$ (the reflection coefficient) equal to zero, we can obtain the significance of $P_{\text {cy }}$ for ETF/INTOR. The temperature and density profiles (Figs. C.I and C.2) we obtained for INTOR $^{3}$ (which should be similar to those for the ETF) using the WHIST ${ }^{4}$ code. The maximum ion and electron temperatures $\left[\mathrm{T}_{i}(0)=68 \mathrm{keV}\right.$ and $\mathrm{T}_{\mathrm{e}}(0)=38.5 \mathrm{keV}$ ] both occur at the plasma center. As seen in Fig. C.3, about $13 \%$ of the energy radiated from the center of the plasma is the result of cyclotron radiation. Using a reflection coefficient of 0.9 would reduce this ratio to $\sim 1$ or $2 \%$. Therefore, a sliding control mechanism in the wall does not seem to be feasible for ETF/INTOR.

However, if $D-D$ plasmas are used so that operating temperatures are higher, it might well be that a sliding shutter mechanism in the wall will be feasible. Also, if the temperature profile is somewhat more peaked than is indicated and if the plasma electron temperature gets above $40 \mathrm{keV}$, then the potential of the sliding shutter concept may increase.

Tamor points out that cyclotron radiation may be a useful diagnostic even if it cannot be used as a control knob.5 A measurement of the spectrum is likely to provide useful detailed information about the plasma capacity and therefore the emissivity and conductivity of the plasma.

\section{REFERENCES}

1. J. F. Etzweiler, J. F. Clarke, and R. H. Fowler, ORNL-TM-4083, Oak Ridge, Tennessee (1973).

2. A. DeBarbieri, Euratom Controlled Fusion Advisory Group on Open Adiabatic Configurations and Radio Frequency Plugging, EUR-CEA-628-AG (1971).

3. S. E. Attenberger (Oak Ridge National Laboratory), private communication, 1979.

4. W. A Houlberg and K. W. Conn, Nucl, Sci. Eng: 64, 141-150 (1.977):

5. S. Tamor, Nuc1. Fúsion 1.8, 229 (1978). 


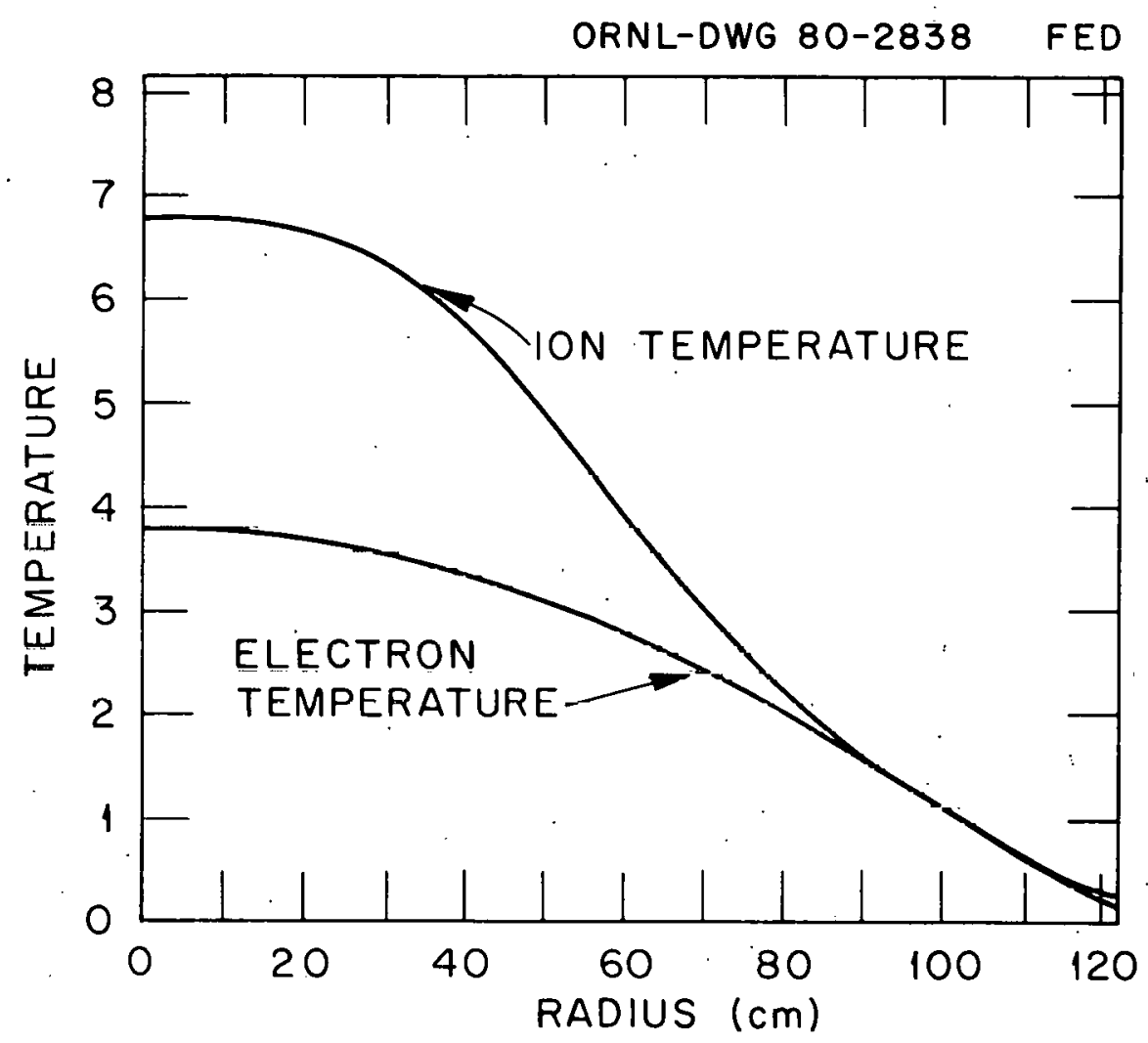

Fig. C.1. Ion and electron tempcraturc profiles. 


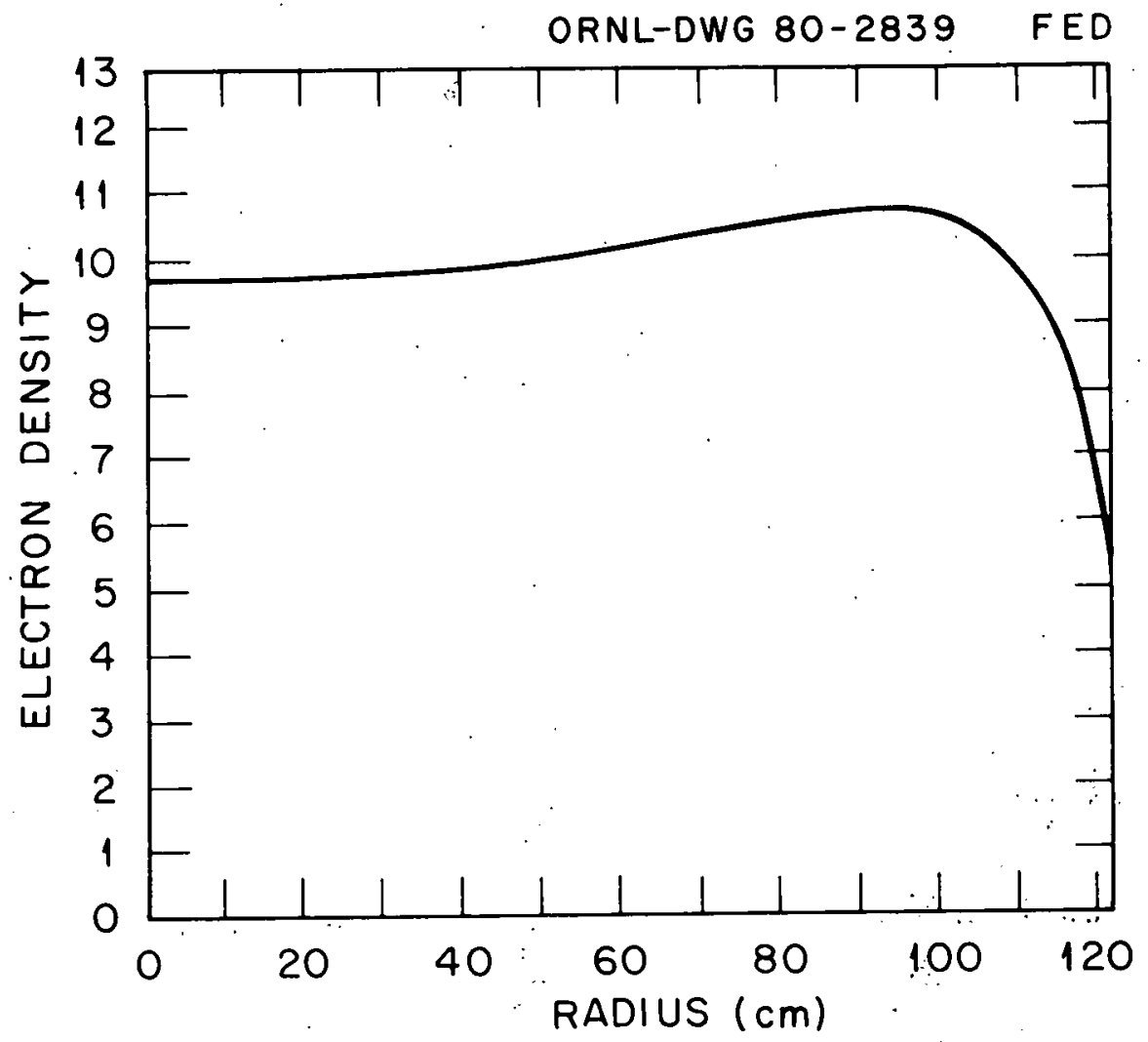

Fig. C.2. Electron density profile for steady-state plasma for INTOR. 


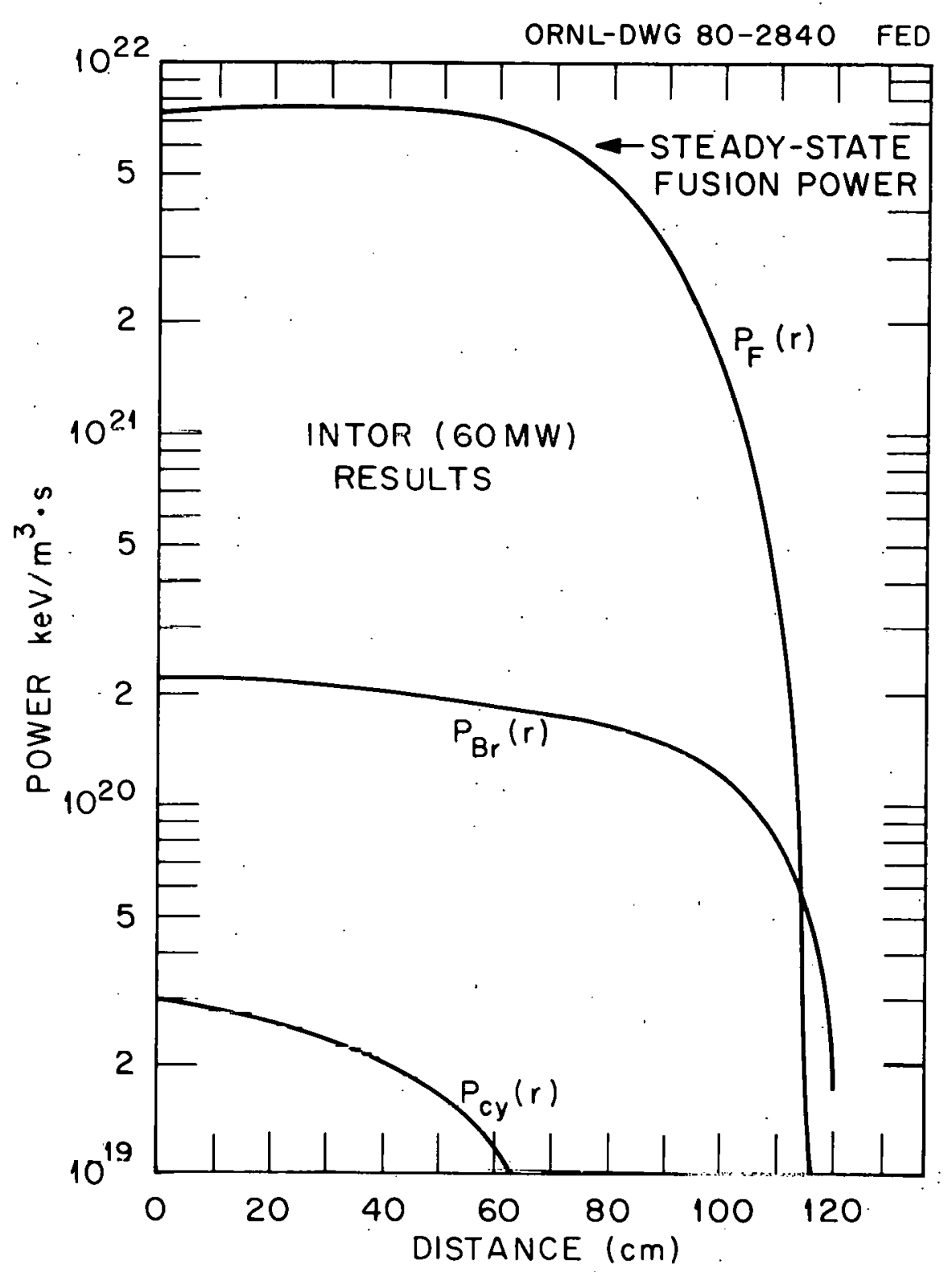

Fig. C.3. Pnwer density as a function of position for INTOR. 
APPENDIX D

THE POSSIBILITY OF EMPLOYING A DIVERTOR

IN A FEEDBACK CONTROL LOOP

\section{D.1 INTRODUCTION .}

A divertor will almost certainly be employed on the ETF. However, a great deal of the physics of divertors is unknown, and many assumptions must be made in modeling the effect of the divertor on the plasma.' The divertor employed on the ETF will probably be a bundle divertor. In a bundle divertor the null point is more or less independent of beta whereas with a poloidal divertor it fluctuates as the plasma current changes. However, because the null point (and therefore the scrape-off thickness) changes with the divertor coils, the null point can be shifted by changing the divertor coil power supply current.

on a power-producing tokamak, the divertor coils probably cannot be placed far enough away from the plasma to use superconducting coils. In that case, copper coils, which require an appreciable fraction of the electrical power produced (5-15\% of the electrical output) will be employed. ${ }^{2}$

In a power-producing reactor the limiting factor that terminates the burn phase of the cycle should probably be volt-seconds rather than impurity buildup. This requires a scrape-off region sufficiently thick to attenuate significantly the charged particle interactions with the wall.

At this point, reasons for and against divertor feedback control can be enumerated. First, reasons for control with a divertor include the following.

(1) The divertor is a sizable piece of equipment, and efficient use of it will probably be cost-effective.

(2) The effective use of the divertor can probably extend the lifetime of the first wall and limiters.

(3) The plasma can maintain a degree of purity probably not possible without divertor control. Helium ash buildup and 1mpurities emanating from the wall may be controlled to some extent, depending on the nature of the diffusion process. 
Reasons for not using a divertor in the feedback loop are as follows.

(1) An interaction exists between the scrape-off thickness and the fueling efficiency of the pellets and gas puffing. The thicker the scrape-off region, the smaller the fraction of pellets that actually get into the plasma region.

(2) It may be that to change the scrape-off region very much requires considerable energy. If a small change in the scrape-off thickness does not change the sputtering rate a great deal, the returns may actually be negative.

An evaluation of the feasibility of divertor control may be premature until the exact type of divertor to be used on the ETF has been selected. However, the possibility of divertor control must be considered from the beginning because of its impact on other systems.

\section{D.2 DIAGNOSTICS FOR DIVERTOR CONTROL}

Divertor control necessitates measuring the scrape-off thickness or measuring the number of particles striking the wall (or limiter) per unit of time. There is little likelihood of directly measuring the scrape-off thickness. However, the detection of impurity radiation appears to be a feasible method of testing the effectiveness of the divertor. Various radiation lines may be viewed from a mirrored port to eliminate neutron streaming. The intensity of the radiation is thus indicative of the scrape-off thickness.

The effectiveness of the divertor depends not only on the scrape-off thickness but also on the particle density and temperature in this region. Up to a point the larger the number of particles, the greater the chance of ionization and the more effective the divertor. If the particle density in the divertor region beçomes greater than the particle density in the plasma edge region, particles will diffuse from the divertor into the plasma region, nullifying the use of the divertor.

The intensity of the impurity radiation is, therefore, a function not only of thickness but also of particle density and temperature. It may be that divertor control can be coupled with the diagnostics by 
varying the gas puff rate rather than by varying the thickness by changing the magnetic field.

\section{REFERENCES}

1. A. Nicolai and A. T. Mense, ORNL/TM-6852, Oak Ridge, Tennessee (1979). 2. W. M. Wells, ORNL/TM-6727, Oak Ridge, Tennessee (1979). 
86

THIS PAGE

WAS INTENTIONALLY

LEFT BLANK 
APPENDIX E

THERMAL CONSEQUENCES TO THE FIRST WALL OF A D-T-FUELED TOKAMAK DUE TO A MAJOR PLASMA DISRUPTION ${ }^{*}$

\section{E.1 INTRODUCTION}

Thermal and magnetic energies stored in an ETF plasma are expected to be as high as $200 \mathrm{MJ}$. The average time for this ETF plasma to become unstable and break up as a result of the disruptive instability $\tau_{D}$ is currently believed to be $0.1-25 \mathrm{~ms}$. During a disruption a tremendous heat flux will occur. The two parameters of central importance in evaluating the thermal consequences of a disruption to the first wall and limiter are the disruption time $\tau_{D}$ and the spatial distribution of the plasma energy on the wall. This, in addition to the presently unknown probability of a MPD occurring during the operating cycle, causes major uncertainties in the first-wall and limiter design of the ETF.

The possibility may exist of obtaining an impending MPD precursor signal and changing the plasma state so that the MPD is averted. However, the consequences to the first wall must still be evaluated so that the number of disruptions before wall failure resulting from thermal stresses may be calculated.

Here we analyze tokamaks with circular and D-shaped plasma cross sections. The center of the plasma need not coincide with the chamber center because of the finite beta shift of the former. Other investigators have examined this problem analytically ${ }^{1,2}$ and numerically ${ }^{3}$ for the circular cross section.

\section{E. 2 MAJOR PLASMA DISRUPTIONS}

Although the complete mathematical description of a MPD is still in the distant future, great steps have been taken in understanding its origin. Because a complete temporal evolution is not yet fuldy understood,

* This paper has been accepted for publication in Nucl. Sci. Eng. 
the breakup pattern and time behavior assumed here are based primarily on experimental observations.

The current theory of MPD's ${ }^{4-6}$ is based on the resistive MHD equations. The $\vec{B}$ field in the plasma is written in terms of a poloidal flux function $\psi^{*}(\vec{r}, \vec{t})$. Because more than one component of the helical flux function is involved, the poloidal flux function is expanded in a Fourier series in the poloidal and toroidal coordinates $\theta$ and $\zeta$,

$\Psi^{*}=\Psi_{\circ \circ}^{*}+\sum_{\mathrm{p}} \sum_{\mathrm{m} / \mathrm{n}-\mathrm{p}} \underset{\mathrm{mn}}{\tilde{*}} \cos (\mathrm{m} \theta+\mathrm{n} \zeta)$

where $p$ is the pitch but not necessarily an integer. The helical flux function $\Psi_{p}$. 15 related to Eq. (F..1) by

$\Psi_{P}=-p\left[\Psi_{o 0}^{*}+\sum_{i m / n=p} \Psi_{m n}^{*} \cos (m \theta+n \zeta)\right]-\frac{1}{2} r^{2}$,

where $r$ is the radial coordinate. Figure E.I is a picture of the $2 / 1$ island (the two islands being represented by the poloidal mode number).

The contours of $\psi_{p}$ for a given $\zeta$ (a poloidal cut) indicate an island structure. The MPD presumably arises when the $2 / 1$ island overlaps with the $3 / 2$ island; with the occurrence of this overlap the fiel. become ergodic, and rapid energy and particle transport can occur. The disruption time is associated with the time for the $2 / 1$ island to grow and for these islands to overlap.

Although the exact breakup pattern is not known, the following experimental observations ${ }^{7}$ provide some insight into the disruption process.

(1) Low values of the safety factor at the limiter increase the probability of a MPD.

(2) Vertical asymmetries may enhance the chance of a MPD.

(3) The disruption seems to propagate from the outside surface inward.

(4) The coupling between the even and odd modes (3/2 and 2/1) appears to be essential for explaining the propagation pattern during the disruption. The resistive terms in the MHD equations become 
ORNL/DWG/FED 78-732R

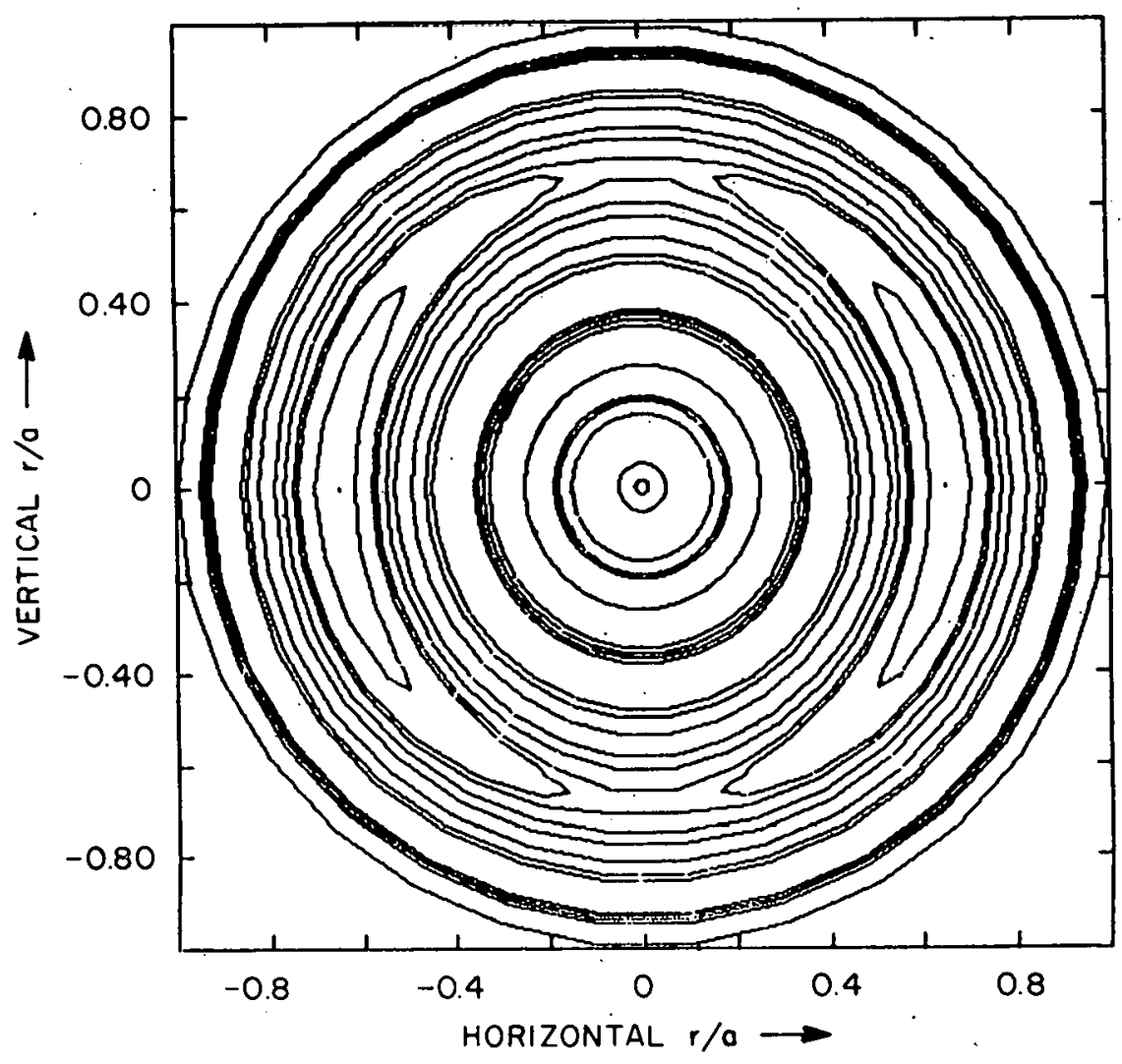

Fig. E.1. Helical flux surface deplcting the $\mathrm{m} / \mathrm{n}=2 / 1$ mode. 
significant near rational surfaces. Islands form and grow generally at rational surfaces with low integers $(1,2,3 / 2, . .$.$) . Thus, the$ current disruption seems to be triggered by a growth of the $m=2$ and $\mathrm{m}=3$ MHD modes.

Various methods have been proposed for suppressing the disruptive instabilities. It seems that broadening the current density profile at the rational surfaces lessens the probability of a MPD; therefore, any way this could be accomplished would be beneficial. (Skin heating of the plasma by neutral gas injection may be one possible way of doing this.) The limitation on plasma density and current in a tokamak is usually imposed by the MPD.

During the disruption the plasma rapidly expands radially; as a result of this rapid expansion much of the plasma will be dumped on the wall. Many particles still follow the field lines and strike the limiter, which will likely be a hot spot.

The disruption time $\tau_{D}$ is difficult to determine because it depends not only on the growth rates of the islands but also on the phenomena that occur after the plasma is in the process of disrupting. A scaling law developed by Carreras et a1. ${ }^{8}$ is based on the observation that the linear $2 / 1$ tearing mode growth time seems to be comparable to the time scale of the nonlinear mode interaction. If plasma diamagnetic effects are ignored, $\tau_{D}$ is shown to be proportional to $S \% / 5 \tau_{R}$ (S is the ratio of the resistive skin time $\tau_{R}\left[\equiv a^{2} /(\mu n)\right]$ and of the time for Alfvén waves to propagate around the torus in the toroidal direction $\tau_{H P}\left[=R_{o}\left(\mu_{0} \rho_{o}\right)^{1 / 2 / B_{\phi O}}\right] ; \rho_{o}$ is the mass density; and $B_{\phi o}$ is the constant toroidal magnetic field). The disruptive time ${ }^{T}$ can be written explicitly as

$\tau_{D}=C\left[\bar{R}_{0}^{2} \bar{m}_{i} \bar{n}_{i} \bar{n}_{\phi o} a^{6}\left(1 / \bar{V}^{3}\right)\right]^{1 / 5}$

where $C$ depends on $q(r)$ and its derivative. The other parameters of Eq. (E.3) are defined in Table E.1, which gives their values for PLT and the ETF. The observed disruption time prevalent in PLT is $\sim_{\mathrm{D}}=500 \mu \mathrm{s}$; 
Table E.1. Parameters used in scaling the disruption time $\tau_{D}$ for ETF

\begin{tabular}{lll}
\hline & PLT & ETF \\
\hline Major radius, $\mathrm{R}_{\mathrm{o}}(\mathrm{m})$ & 1.3 & 5.0 \\
Ion mass, $\overline{\mathrm{m}}_{\mathrm{i}}$ (proton mass) & 2.0 & 2.5 \\
Ion density, $\overline{\mathrm{n}}_{\mathrm{i}}\left(10^{\left.13 \mathrm{~cm}^{-3}\right)}\right.$ & 10 & 13 \\
Toroidal field, $\overline{\mathrm{B}}_{\phi 0}(\mathrm{~T})$ & 5 & 5.3 \\
Minor radius, $\overline{\mathrm{a}}(\mathrm{m})$ & 0.45 & 1.25 \\
Voltage at limiter, $\overline{\mathrm{V}}(\mathrm{V})$ & $2-4$ & $0.05-0.1$ \\
\hline
\end{tabular}

from this value and the scaling law given by Eq. (E.3), a disruption time of $\tau_{D}=24 \mathrm{~ms}$ is projected for the ETF. (This extrapolation is shown in Fig. E.2.)

\section{E. 3 METHODOLOGY}

The sequence of calculational steps for analyzing the impact of a MPD on the first wall is shown in Fig. E.3. Pecause the first wall is closest to the plasma, it will be most directly affected by the plasma disruption. As the plasma current dies away and the heat flux strikes the wall, eddy currents will be induced in the first wall and will add to the total heat load. We assume that the wall is 316 stainless steel, and we see the thermal properties reported by Kim. ${ }^{9}$

The interaction of the plasma disruption with the wall is initially treated as though no other component of the reactor were involved. The heat load on the wall is due to the surface heat flux and the induced eddy currents generating a volumetric heat rate of $\mathrm{I}_{\mathrm{w}}^{2} \mathrm{R}_{\mathrm{w}} / \mathrm{V}_{\mathrm{w}}$. A lumped parameter model is used to estimate this heat rate, which is then used as a source for obtaining the wall temperature distribution.

Several assumptions are made in this calculation.

(1) The mutual plasma wall inductance $\mathrm{L}_{12}$ and the plasma self-inductance $\dot{L}_{11}$ are constant during the plasma disruption process and then become zero when the plasma current goes to zero. 


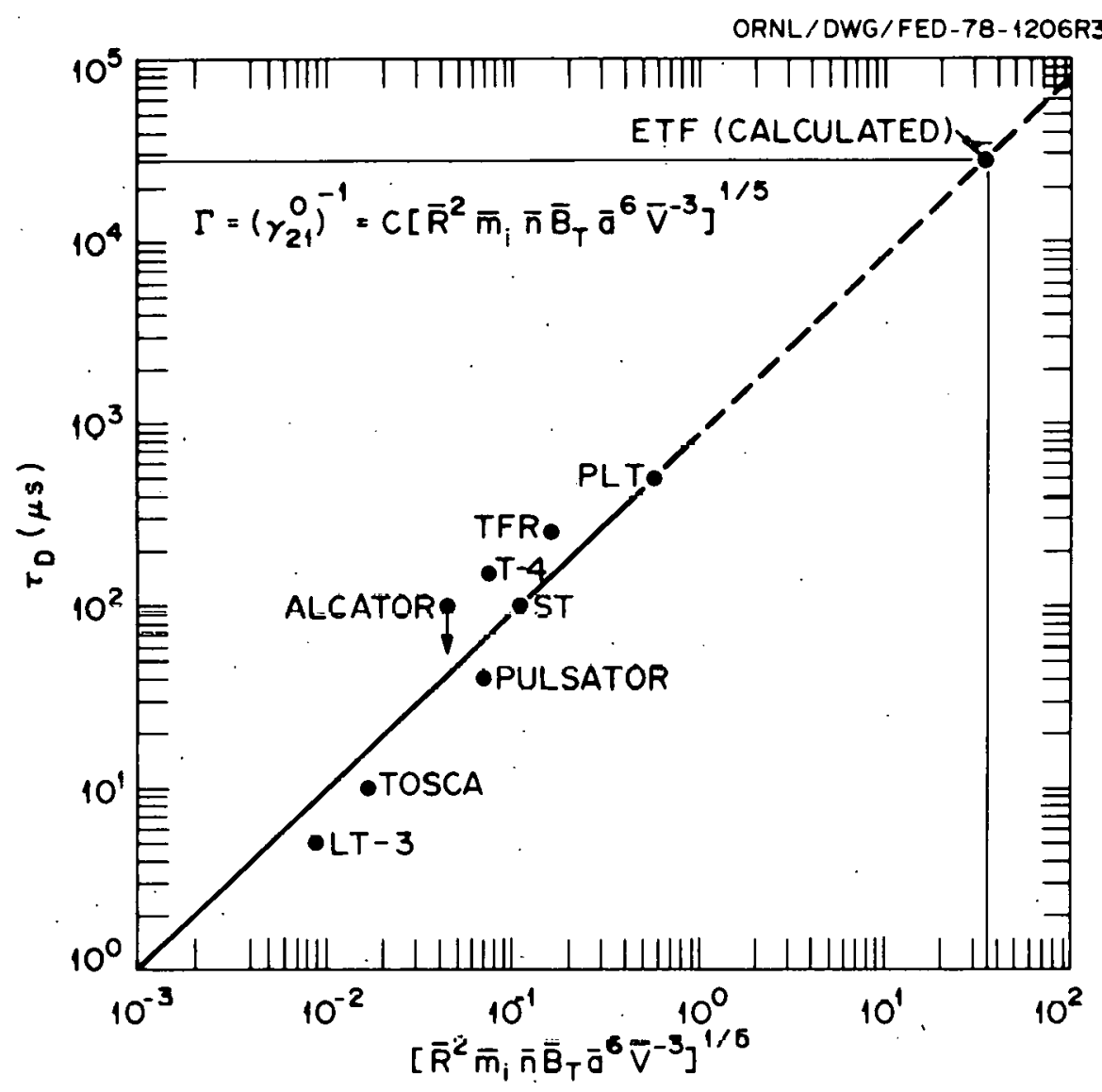

Fig. E.2. Scaling law for obtaining the disruption time for the ETF. 


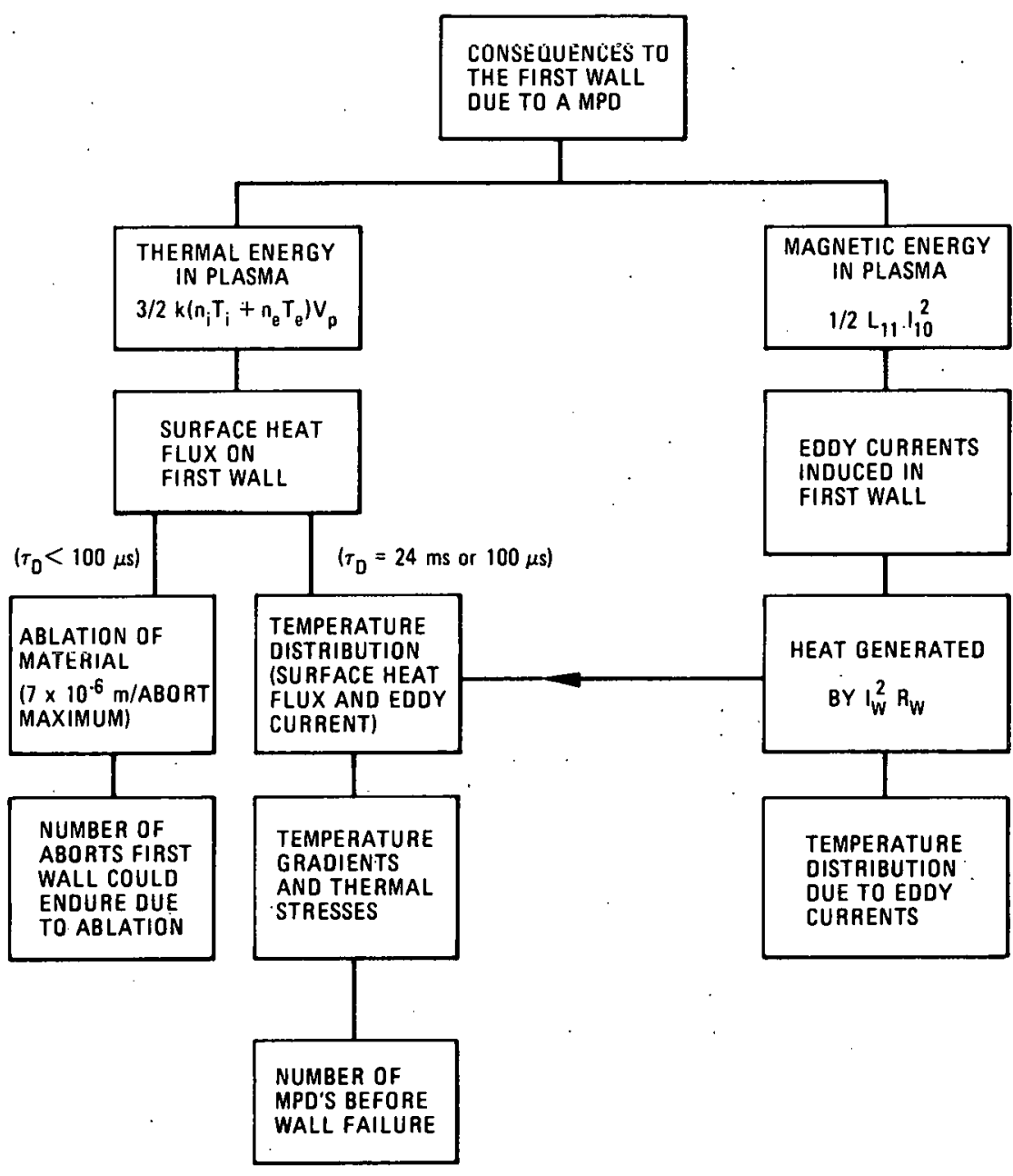

Fig. E.3. Schematic diagram characterizing the methodology in treating the consequences to the first wall. 
(2) The Biot number is small;

$B i=\frac{\text { surface convection heat transfer rate }}{\text { internal conduction heat transfer rate }}=\frac{h_{x} \delta}{k_{w}}$

is less than unity. This means that the temperature profile in the wall is nearly flat.

(3) The Fourier modulus is large (Fo $>1$ ) and is defined as

$F_{0}=\frac{\alpha_{\mathrm{r} I} \tau_{n}}{\delta_{\mathrm{W}}^{2}}=\left(\frac{\text { distance temperature wave travels }}{\text { wall thickncss }}\right)^{2}$,

where $\alpha_{w}$ is the wall thermal diffusivity. It is obvious that each of these assumptions will be violated at least some time during the disruption.

E.4 THE SURFACE HEAT FLUX AND VOLUMETRIC-HEAT GENERATION RATE FOR THE CIRCULAR CHAMBER

Table E.2 lists various parameters important in estimating the surface heat flux and volumetric heat generation rate resulting from eddy currents in the wall; we ignore heat generation in the wall resulting from ncutron capture.

Figure E.4 is a schematic diagram of the physical system to be simulated. Following are four primary equations that are in the lumped parameter model. The energy balance equation for the wall is

$\frac{d E}{d t}=q_{w e}^{\prime \prime} A_{w}+q_{w E}^{\prime \prime \prime} V_{w}-U A_{w}\left(T_{w}-T_{0}\right)$

The voltage balance equation for eddy currents in the wall is

$$
L_{22} \frac{d I_{2}}{d t}+R_{2}\left(T_{w}\right) I_{2}(t)=v_{2}
$$


Table E.2. Typical parameter values for $\operatorname{ETF}^{a}$

\begin{tabular}{|c|c|}
\hline Quantity & Value \\
\hline Ion density (average), $\mathrm{n}$ & $1.3 \times 10^{20} \mathrm{~m}^{-3}$ \\
\hline Average ion temperature, $\mathrm{T}_{i}$ & $13.0 \mathrm{keV}$ \\
\hline Minor radius, a & $1.25 \mathrm{~m}$ \\
\hline Major radius, $R_{o}$ & $5.0 \mathrm{~m}$ \\
\hline Elongation, $\sigma$ & 1.6 \\
\hline First-wall area, $A_{w}$ & $348.6 \mathrm{~m}^{2}$ \\
\hline First-wall thickness, $\delta_{w}$ & $1.65 \times 10^{-3} \mathrm{~m}$ \\
\hline Plasma volume, $V_{p}$ & $227.4 \mathrm{~m}^{3}$ \\
\hline Plasma resistance, $R_{E}$ & $\begin{array}{l}3.0 \times 10^{-9} \Omega \text { at } \\
13.0 \mathrm{keV}\end{array}$ \\
\hline Toroidal field at wall, $B_{\phi \omega}$ & $7.7 \cdot \mathrm{T}$ \\
\hline Steady-state plasma current, $I_{p o}\left(I_{10}\right)$ & $5.6 \times 10^{6} \mathrm{~A}$ \\
\hline Heat energy in plasma, $E_{t p}$ & $1.85 \times 10^{8} \mathrm{~J}$ \\
\hline Neutron wall loading; $q_{n}^{\prime \prime}$ & $2.38 \times 10^{6} \mathrm{~W} / \mathrm{m}^{2}$ \\
\hline Self-inductance of plasma, $L_{11}$ & $12.2 \times 10^{-6} \mathrm{H}$ \\
\hline Self-inductance of wall, $\mathrm{L}_{22}$ & $9.75 \times 10^{-6} \mathrm{H}$ \\
\hline Steady-state surface heat filux, $q_{\text {to }}^{\prime \prime}$ & $5.94 \times 10^{5} \mathrm{~W} / \mathrm{m}^{2}$ \\
\hline
\end{tabular}

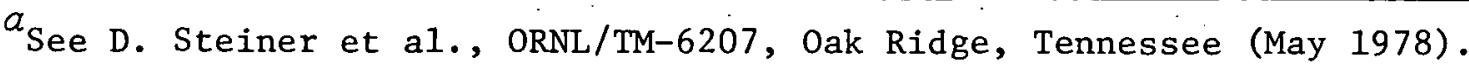

The voltage induced in the wall by plasma decay current is

$V_{2}=-I_{12} \frac{d I_{1}(t)}{d t}$

The plasma current as a function of disruption time is assumed to be of the form.

$I_{1}(t)=I_{10} \exp -\left(t / \tau_{D}\right)$

In these equations $V_{2}$ is the voltage induced in the wall; $R_{2}\left(T_{w}\right)$ is the wall electrical resistance, which changes with wall temperature; 


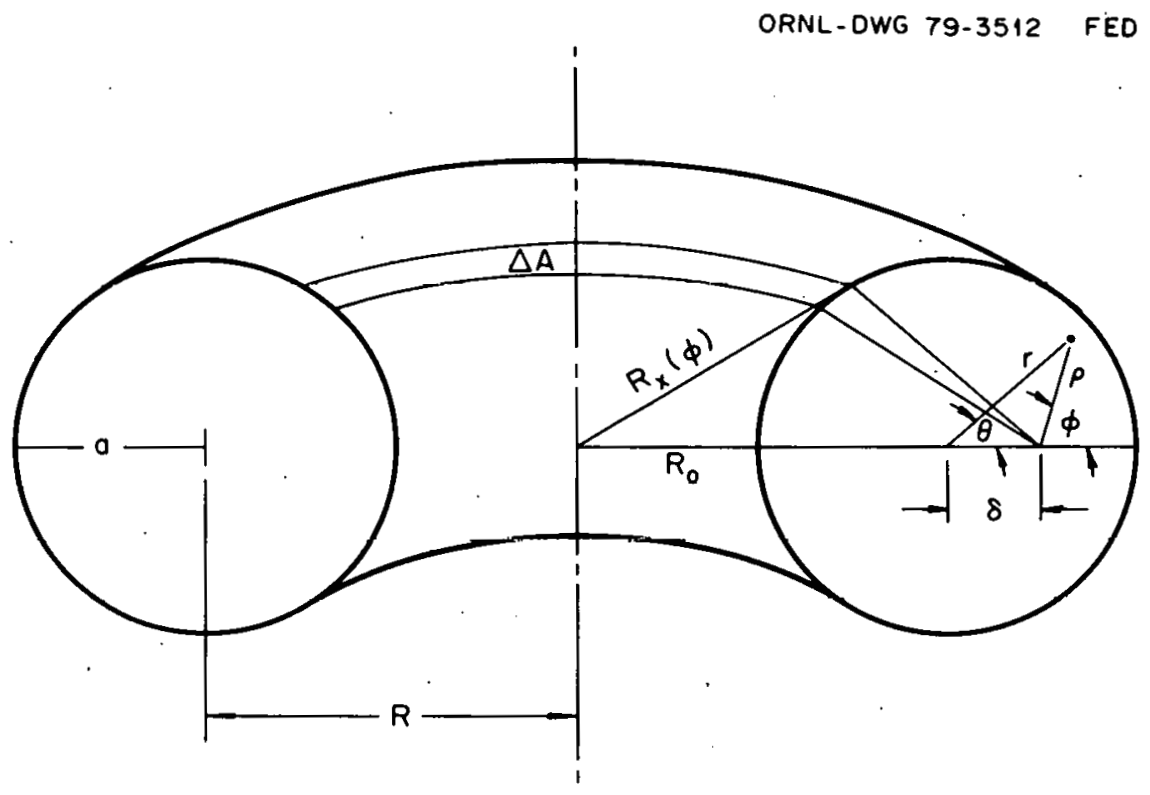

Fig. E.4. Schematic diagram for analysis of the plasma/wall interaction. 
$V_{w}=A_{w} \delta_{w}$ is the volume of the wall; $q_{w E}^{\prime \prime \prime}(t)=I_{2}^{2}(t) R_{w}\left(T_{w}\right) / V_{w}$ is the volumetric heat generation rate; $\mathrm{I}_{10}$ is the steady-state plasma current; $\mathrm{U}$ is the overall heat transfer coefficient; $\mathrm{T}_{0}$ is the temperature of the coolant; $E_{w}=$ total thermal energy of the wall; and $I_{2}$ is the wall current.

The surface heat flux $q_{w t}^{\prime \prime}(t)$ is obtained in a manner illustrated in Fig. E.5. Because the plasma center may not coincide with the vessel center, a coordinate transformation is necessary. The degree of offcenteredness is a function $\beta_{\theta}$ (poloidal $B$ ).

A distribution describing the temperature and density profiles prior to and during the disruption is developed by assuming a functional form that satisfies known boundary conditions. The plasma's thermal energy is then computed using kinetic theory based on this distribution. As the disruption continues plasma particles are assumed to travel outward with a constant radial velocity; thus, $\partial r / \partial t=v$ and $\partial \theta / \partial t=0$. The center of the distribution rather than the center of the circle is taken as the origin of the coordinate system. Figure E.5 illustrates the geometric relationship between the two systems. The transformation equations relating these coordinate systems are

$\rho^{2}=r^{2}+\delta^{2}-2 \delta r \cos \theta$,

$r^{2}=\rho^{2}+\delta^{2}+2 \delta \rho \cos \theta$

and

$\rho \sin \phi=r \sin \theta$.

To ensure equality of volume elements the Jacobian of the transformation is calculated as

$J=\left|\begin{array}{l}\frac{\partial r}{\partial \rho} \frac{\partial r}{\partial \phi} \\ \frac{\partial \theta}{\partial \rho} \frac{\partial \theta}{\partial \phi}\end{array}\right|=\sqrt{\rho^{2}+\delta^{2}+2 \delta \rho \cos \theta}$. 
ORNL-DWG 79-3511 FED
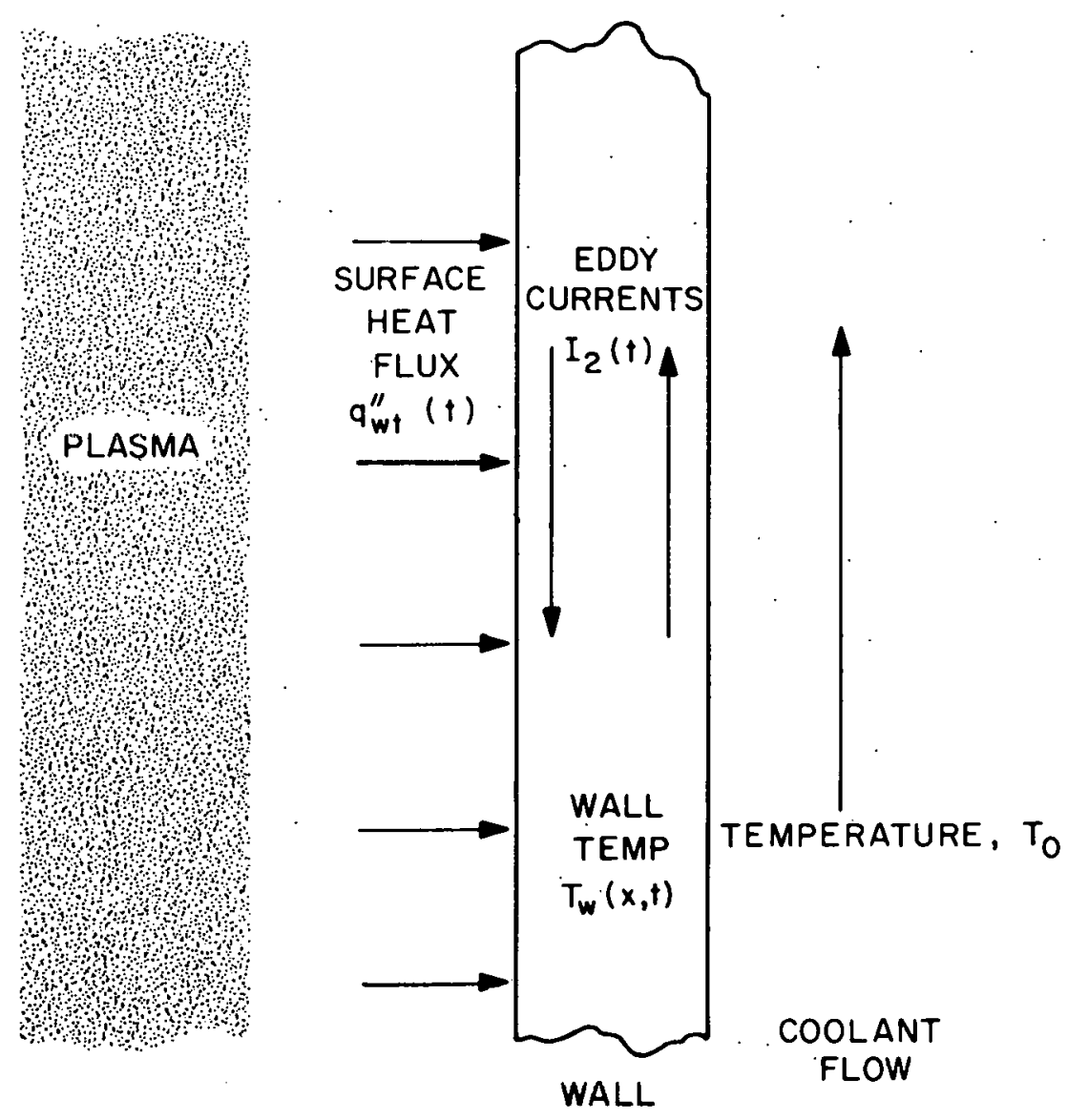

(316 STAINLESS STEEL)

Fig. E.5. Geometric relationships between the $(\rho, \phi)$ and $(r, \theta)$ systems. 
If we assume the distribution function to be parabolic in $r$ with a maximum at the magnetic center of the reactor, we have

$F(\rho, \phi)=1-\left[\frac{\rho}{\rho a(\phi)}\right]^{2}$

Here $\rho_{a}$ is the distance from the center of the distribution to the reactor first wall at a particular $\phi$ and is given by

$\rho_{a}(\phi)=-\delta \cos \phi+\sqrt{\delta^{2} \cos ^{2} \phi-\left(\delta^{2}-a^{2}\right)}$.

The density and temperature distributions can be written as

$n(r, \theta)=n_{0} F(\rho, \phi)$

and

$T(r, \theta)=T_{0} F(\rho, \phi)$,

where the maximum density and temperature, $\mathrm{n}_{0}$ and $\mathrm{T}_{0}$, can be evaluated knowing the average density and temperature as given in lable E.2. An alternative approach would be to normalize with respect to power rather than to density and temperature independently.

The first-wal.]. surface heat flux $q_{w t}^{\prime \prime}(t)$ is estimated by assuming that the plasma moves out radially at a constant velocity. By definition, the total thermal energy of the plasma is deposited on the wall in a disruption time. $\tau_{D}$. Because of the asymmetry in $\phi$ some portions of the wall will still be bombarded with particles while no energy is being deposited on other sections. Thus, the disruption time is

$\tau_{D}(\phi)=\frac{\rho_{a}(\phi)}{v}$ 
where $\rho_{a}(\phi)$ is defined by Eq. (E.12) and $v$ is the velocity (constant) of the particles resulting from a MPD.

The thermal energy in the plasma is given by

$E_{p t}=\frac{3}{2}\left(n_{i} T_{i}+n_{e} T_{e}\right) V_{p}$,

where $E_{p t}$ is the total thermal energy. in the plasma, $n_{e}$ and $n_{i}$ are the average electron and ion densities, $T_{e}$ and $T_{i}$ are the electron and ion temperatures, and $\mathrm{V}_{\mathrm{p}}$ is the volume of the plasma. Assuming equal electron and ion temperatures, Eq. (E.16) beçomes

$E_{p t}=3 n_{0} T_{0} V_{p} \int_{0}^{2 \pi} \int_{0}^{\rho_{a}(\phi)} \frac{\rho}{\tau_{D}(\phi) v}[F(\rho, \phi)]^{2} d \rho d \phi$.

The energy in an element of ring at radius $\rho$ and angle $\phi$ with thickness $\mathrm{d} \rho$ about $\mathrm{d} \phi$ is

$d E_{p t}=3 n_{o} T_{o}[F(\rho, \phi)]^{2} 2 \pi R_{x} \rho d \rho d \phi$,

where $R_{x}(\phi)$ is the radius from the center of the torus to the elemental ring (illustratcd in Fig. E.5).

It has been assumed that the ring, defined by Eq. ( $H .18$ ), is moving out radially at a constant velocity. Thus, the rate at which energy is otriking the wall is

$\frac{d F_{p t}}{d t}=\frac{\partial F p t}{\partial \rho} \cdot \frac{d r}{d t}+\frac{\partial F_{1}}{\partial \phi} \cdot \frac{d \phi}{d t}=P_{N S} t[F(t, \phi)]^{2} d \phi$,

where

$\mathrm{P}_{\mathrm{NS}}=6 \pi \mathrm{n}_{\mathrm{o}} \mathrm{T}_{\mathrm{o}} \mathrm{v}^{2} \mathrm{R}_{\mathrm{x}}(\phi)$ 
The surface heat flux incident on a segment of the first wall between $\phi_{i}$ and $\phi_{i+1}$ is found from Eq. (E.19) to be

$$
q_{w t}^{\prime \prime}(t, \phi)= \begin{cases}q_{\text {to }}^{\prime \prime} & t<0 \\ \frac{1}{\left(\phi_{i+1}-\phi_{i}\right)} \int_{\phi_{i}}^{\phi_{i+1} \frac{P_{N S}}{D A}[F(t, \phi)]^{2} d \phi+q_{t o}^{\prime \prime} \exp \left(-t / \tau_{D}\right),} \\ q_{\text {to }}^{\prime \prime} \exp \left(-t / \tau_{D}\right) & 0 \leqslant t \leqslant \tau_{D}\end{cases}
$$

where DA corresponds to the wall area subtended by the element of angle $\phi_{i+1}$ and $\phi_{i}$ (shown in Fig. E.5).

Figure E. 6 indicates how the vacuum vessel was segmented for solving Eqs. (E.4)-(E.7). These angles are all 36 , and arc 1 corresponds to the outside wall of the lokaniak.

\section{E.5 RESULTS OF THE LUMPED PARAMETER MODEL}

A graphic display of the wall distribution function, current, and temperature appears in Figs. E.7-E.9, which are for a $\delta / a$ of 0.3 ; each figure shows the parameter of interest at the five arcs. The maximum temperature rise always occurs in arc 5 , followed by $\operatorname{arcs} 4,3,2$, and 1 , respectively. This is to be expected because as $\delta / a \rightarrow 1$, a greater Eraction of the plasma's thermal energy resides in the higher-numbered arcs. The effective disruption time also increases with the arc number as $\delta / a$ increases because the wall is bombarded for a longer period of time.

The wall current curves show that as $\delta / a$ increases, the maximum wall current peaks increasingly higher in arc 1 , which is consistent with Intuition because the induced eddy current in the wall goes as the plasma current density. As can be seen from the plots the separation between these induced currents increases with $\delta / a$. 

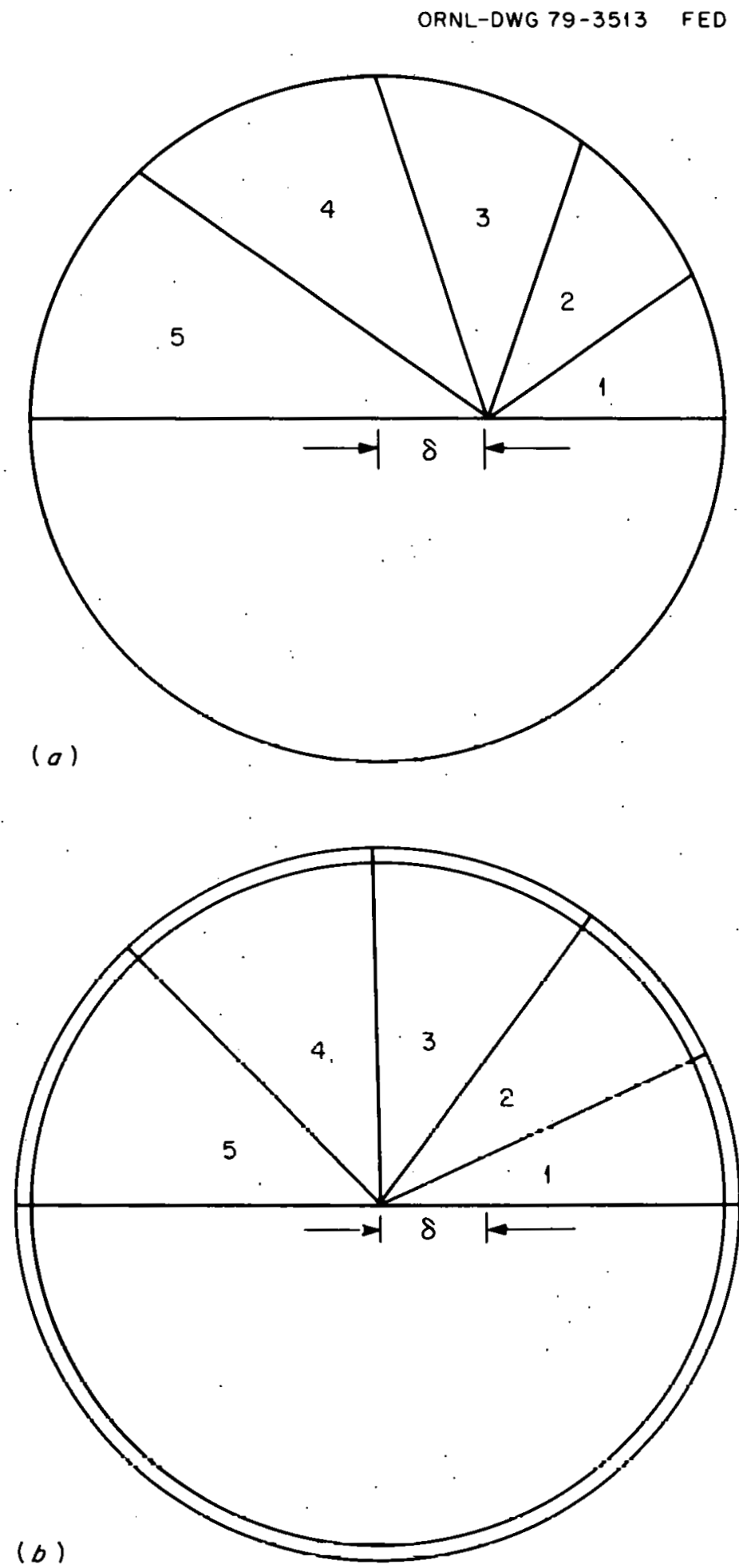

Fig. E. 6(a). Wall arcs in the $(\rho, \phi)$ system for lumped parameter analysis. (b) Wall arcs in the $(r, \theta)$ system for distributed parameter analysis. 


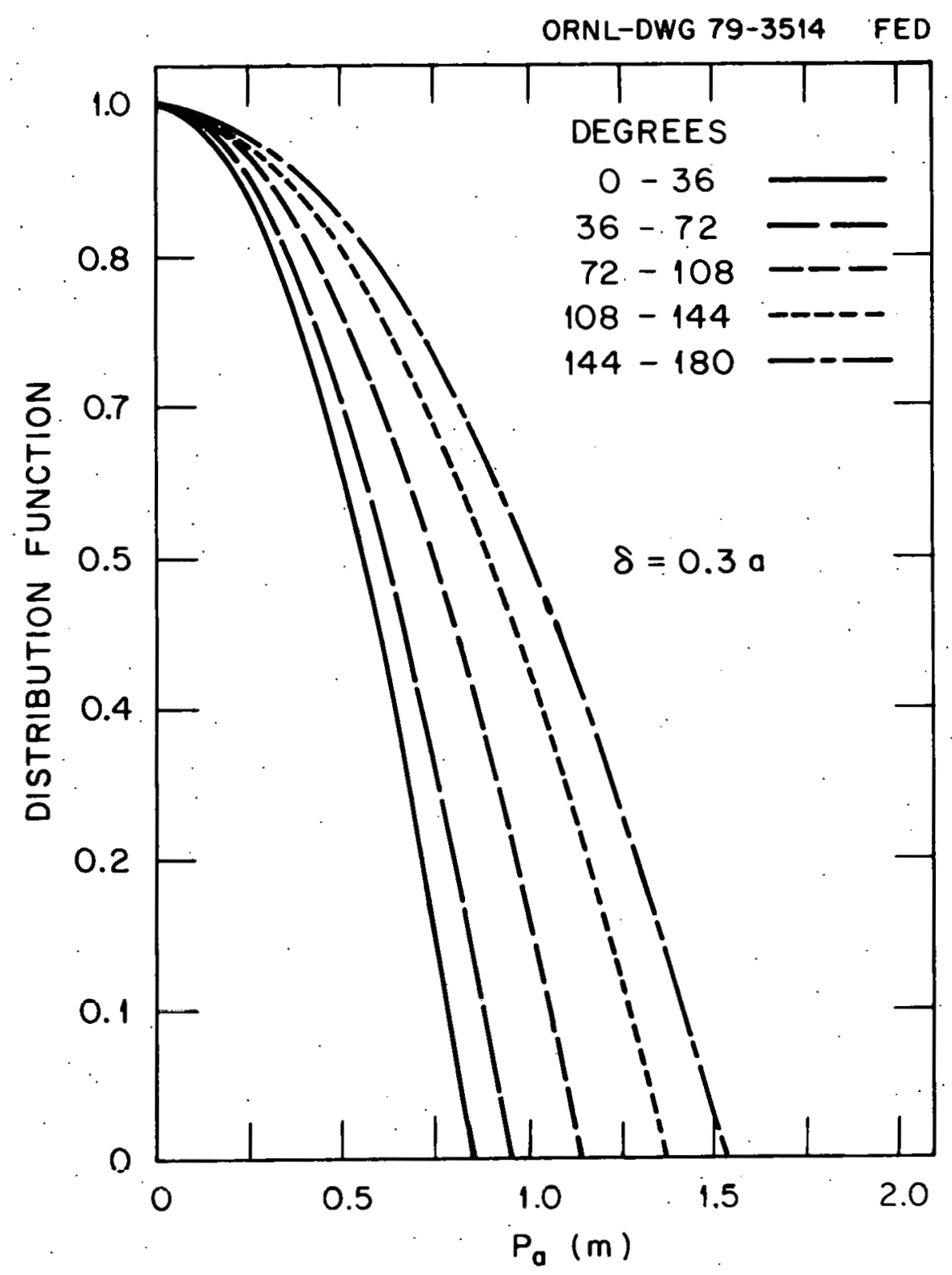

Fig. E.7. The plasma distribution vs position. 


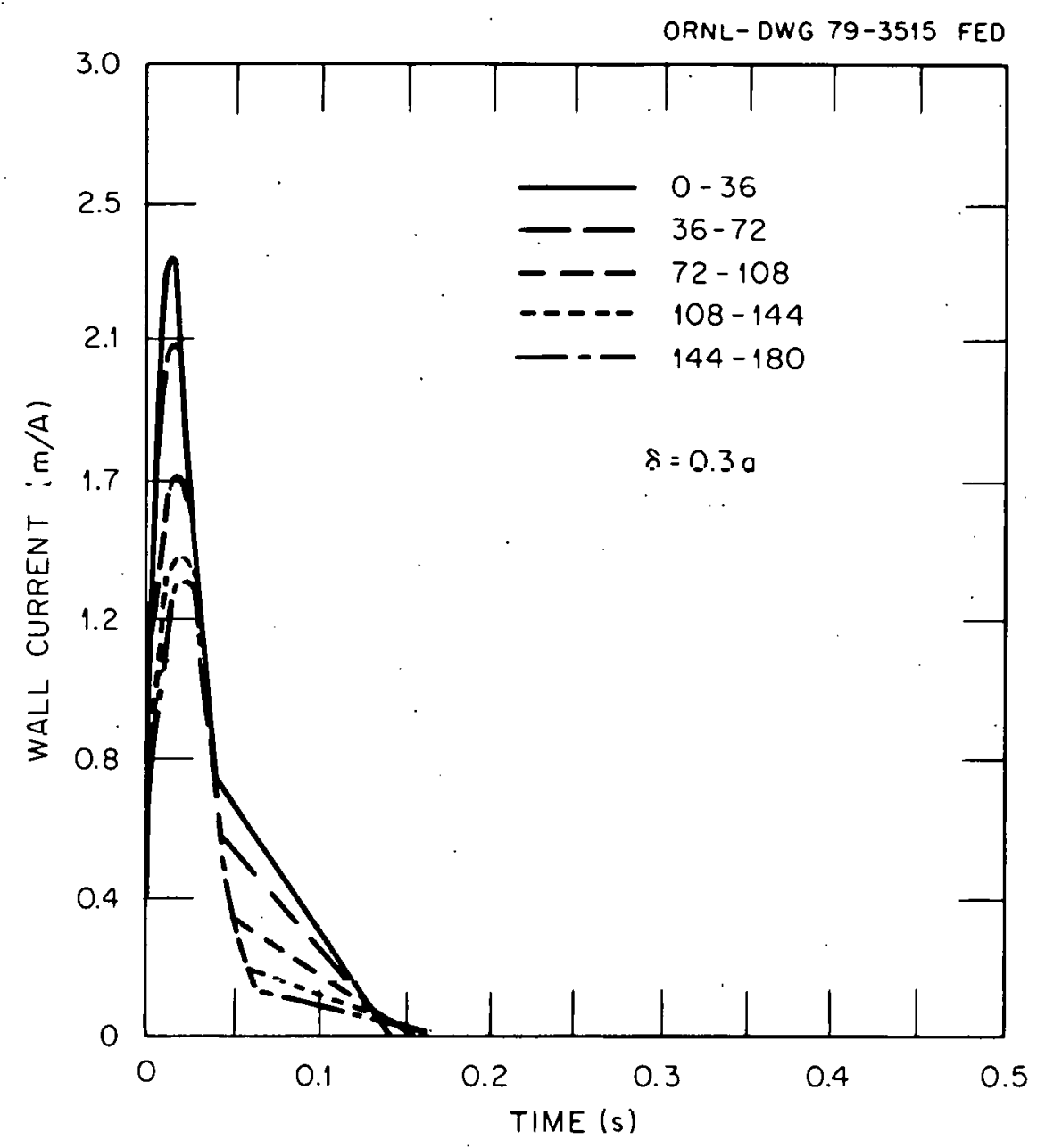

Fig. E.8. Wall current vs time. 


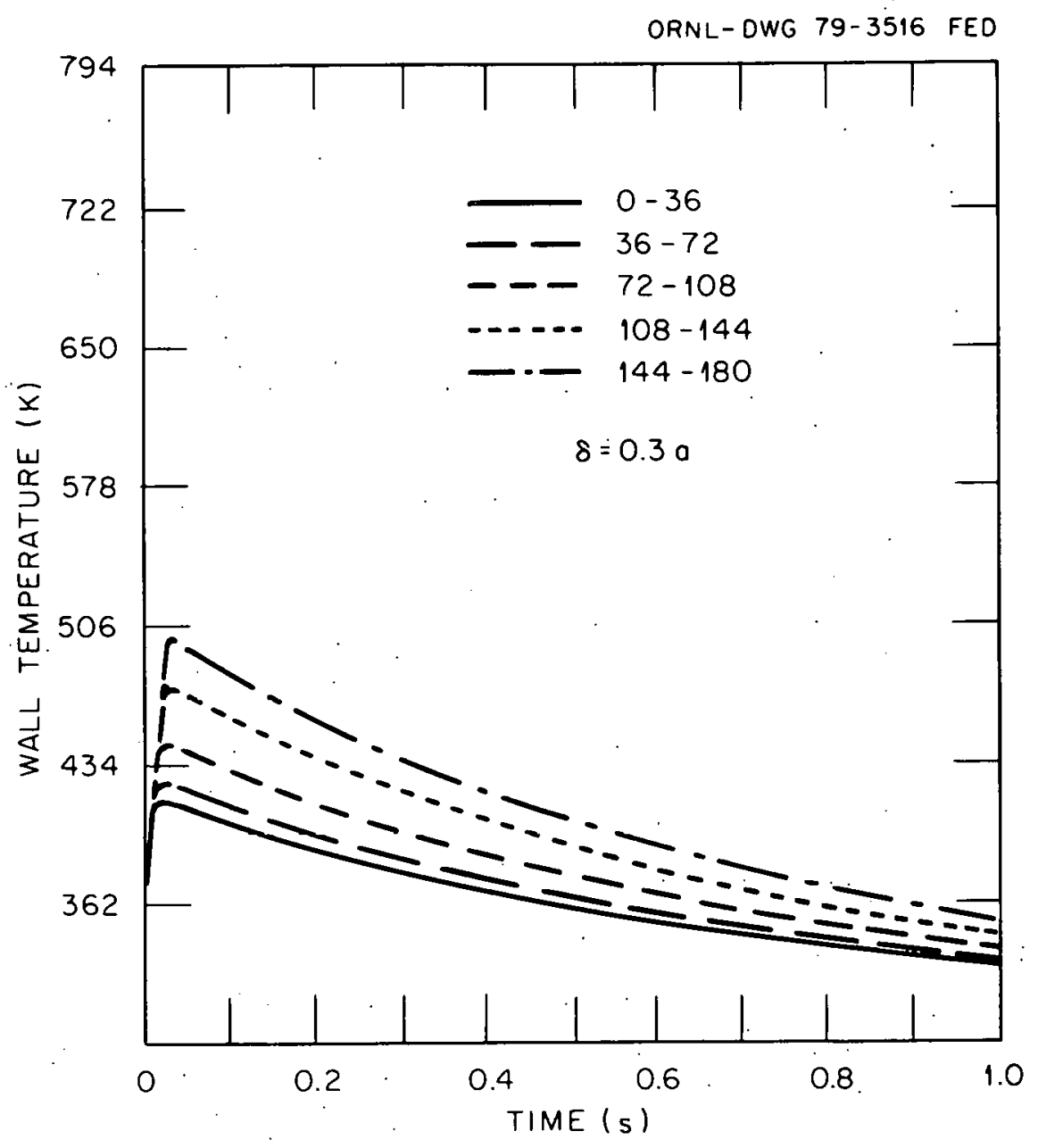

Fig. E.9. Wall temperature vs time. 
The analysis indicates that the heating effect is greatest on the interior of the torus, arc 5. This is primarily due to the surface heat flux. A summary of temperature and wall current data for various $\delta$ 's appears in Table E.3.

The temperature difference between the maximum and minimum temperature increases with $\delta / a$. In the worst case, when $\delta \cong a$, this temperature difference is $270 \mathrm{~K}$. The time at which the maximum temperature occurs also increases with $\delta$. This most probably results from a lengthening of the effective disruption time.

Un the other hand, the time at which the maximum current is achieved decreases with increasing $\delta / a$. The induced potential in the wall is proportional to the time derivative of the plasma current. As $\delta \rightarrow$ a the magnitudes of $\mathrm{dI}_{1} / \mathrm{dt}$ increase; thus, a higher wall current is achieved in a shorter time span. Also, the mutual inductance between the wall segment of arc 1 and the plasma increases with $\delta$; therefore, two factors act to increase the wall current in arc 1 . The same arguments apply to arc 5 only in the opposite sense; thus, the current is lowered in arc 5.

Plots of the distribution function show less and less plasma in the solid angle identified with arc 1 as $\delta / a \rightarrow 1$. This fact coincides with the peaking of the wall current in arc 1 . The temperature versus time plots show that the temperature rise decreases to almost zero as $\delta / a \rightarrow 1$. Thus, by qualitative arguments one can state that the effect of Joule hearing is small compared to the surface heat flux.

Two other parameters of interest are the fraction of magnetic energy stored in the plasma that is converted into heat and the fraction of magnetic and thermal energy that goes into heating the wall. Defined mathematically, these are, respectively.

$f_{e t}=\frac{\int_{0}^{2 \pi} \int_{0}^{\infty} I_{2}^{2}(t) R_{2}(T) d t}{1 / 2 L_{1 I} I_{i, 0}^{2}}$

and 
Table E.3. A summary of results for several $\delta / a^{\prime} s$

\begin{tabular}{|c|c|c|c|c|c|c|}
\hline $\begin{array}{c}\delta / \mathrm{a} \\
(\mathrm{m} / \mathrm{m})\end{array}$ & $I_{\max } i I_{\min } a$ & $I_{\max }$ & $\begin{array}{l}\text { Time maximum } \\
\text { current occurs } \\
\text { (ms) }\end{array}$ & $\underset{(\mathrm{K} / \mathrm{K})}{\mathrm{T}_{\max } / \mathrm{T}_{\min }}$ & $\mathrm{T}_{\mathrm{max}}^{\mathrm{T}}$ & $\begin{array}{l}\text { Time maximum } \\
\text { temperature occurs } \\
\text { (ms) }\end{array}$ \\
\hline 0 & 1.07 & 1.67 & 18.6 & 1.0 & 454 & 23.0 \\
\hline 0.1 & $1: 16$ & 1.83 & 18.2 & 1.07 & 469 & 28.1 \\
\hline 0.3 & 1.75 & 2.42 & 16.8 & 1.20 & 502 & 29.3 \\
\hline 0.5 & 2.82 & 3.15 & 12.2 & 1.35 & 539 & 33.2 \\
\hline 0.7 & 5.50 & 4.12 & 7.4 & 1.51 & 571 & 37.2 \\
\hline 0.977 & 27.20 & 5.50 & 2.0 & 1.75 & 637 & 42.3 \\
\hline
\end{tabular}

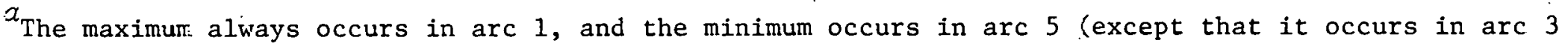
when $\delta / a=0)$.

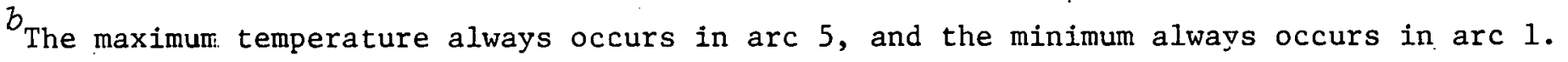


$f_{t t}=\frac{\int_{0}^{2 \pi} \int_{0}^{\tau(\phi)}\left[1 / \tau_{D}(\phi)\right] C(T) \rho_{w}(T) V\left[T(t)-T_{i}\right] d t d \phi}{E_{p t}+1 / 2 L_{11} I_{10}^{2}}$.

The mass density of the wall is $\rho$.

Because the eddy currents die away exponentially, Eq. (E.22) is integrated to infinity in time; however, Eq. (E.23) is integrated only to the disruption time. During the disruption the plasma's energy acts to raise the wall's internal energy while the coolant works to reduce it. Beyond the disruption time the coolant acts to lower the wall's internal energy. A more exact expression would include heat due to ohmic heating beyond the disruption time, which is neglected in our calculation. The value of $\mathrm{E}_{\mathrm{pt}}$, determined using Eq. (E.16), is $1.85 \times$. $10^{6} \mathrm{~J}$ when $\delta=0$.

The fraction of magnetic and thermal energy that goes into heating the wall $\mathrm{f}_{t t}$ is not significantly dependent on $\delta$. However, $f_{\text {et }}$ increases by almost a factor of 2 as $\delta / a$ goes from zero to nearly one. Values of $\mathrm{f}_{\mathrm{tt}}$ and $\mathrm{f}_{\mathrm{et}}$ corresponding to the $\delta / \mathrm{a}^{\prime} \mathrm{s}$ considered appear in Table $\mathrm{E} .4$.

Table E.4. A summary of $f_{e t}$ and $f_{t t}$ as a function of $\delta / a$

\begin{tabular}{lll}
$\begin{array}{c}\delta / \mathrm{a} \\
(\mathrm{m} / \mathrm{m})\end{array}$ & $\mathrm{f}_{\mathrm{et}}$ & $\mathrm{f}_{\mathrm{tt}}$ \\
\hline 0 & 0.304 & 0.310 \\
0.1 & 0.318 & 0.312 \\
0.3 & 0.356 & 0.312 \\
0.5 & 0.402 & 0.310 \\
0.7 & 0.468 & 0.316 \\
0.977 & 0.596 & 0.308 \\
\hline
\end{tabular}

A parabolic distribution function was assumed in this analysis. The sensitivity of the results can be obtained by using other distribution functions. The analysis was therefore done at selected $\delta / a$ 's using the alternative distributions 
$F(\rho, \phi)=\left\{1-\left[\frac{\rho}{\rho_{a}(\phi)}\right]^{2}\right\}^{1 / 2}$

and

$f(\rho, \phi)=\cos \left[\frac{\pi}{2} \frac{\rho}{\rho_{a}(\phi)}\right]$.

A comparison of the results using Eqs. (E.24), (E.25), and (E.11) for the same $\delta / a$ is found in Table E.5. The comparison indicates that the results of the lumped parameter model are only mildly sensitive to the distribution chosen.

\section{E.6 TEMPERATURE DISTRIBUTION IN THE CIRCULAR FIRST WALL}

A 2-D analysis of the temperature profile was made with the HEATING5 10 code. The temperature gradient in the first wall during the plasma disruption was found and then used to obtain the thermal fatigue resulting from the discruption.

* Three contributions to the heat load in the first wall are considered:

(1) the surface heat flux impinging on the wall as the plasma disrupts,

(2) Joule heating resulting from the eddy currents induced on the wall,

(3) neutron flux interacting with the stainless steel, which causes a heat load internal to the wall; the neutron flux heating contribution is terminated after the steady-state temperature distribution is achieved.

Although electrons with very high energy ( $>1 \mathrm{MeV}$ ) can also contribute to the internal energy generation, these are neglected, as are any $x$ rays emanating from the plasma. Disruption times of $100 \mu \mathrm{s}$ and $24 \mathrm{~ms}$ are considered for $\delta / a=0$ because of the uncertainty in the disruption time. Because the temperature gradient for the 100- $\mu$ s case is very steep, the wall is divided into two regions. The region close to the plasma has many more nodes than the region near the coolant. 
Table E.5. A comparison of the lumped parameter nodel using different distribution functions

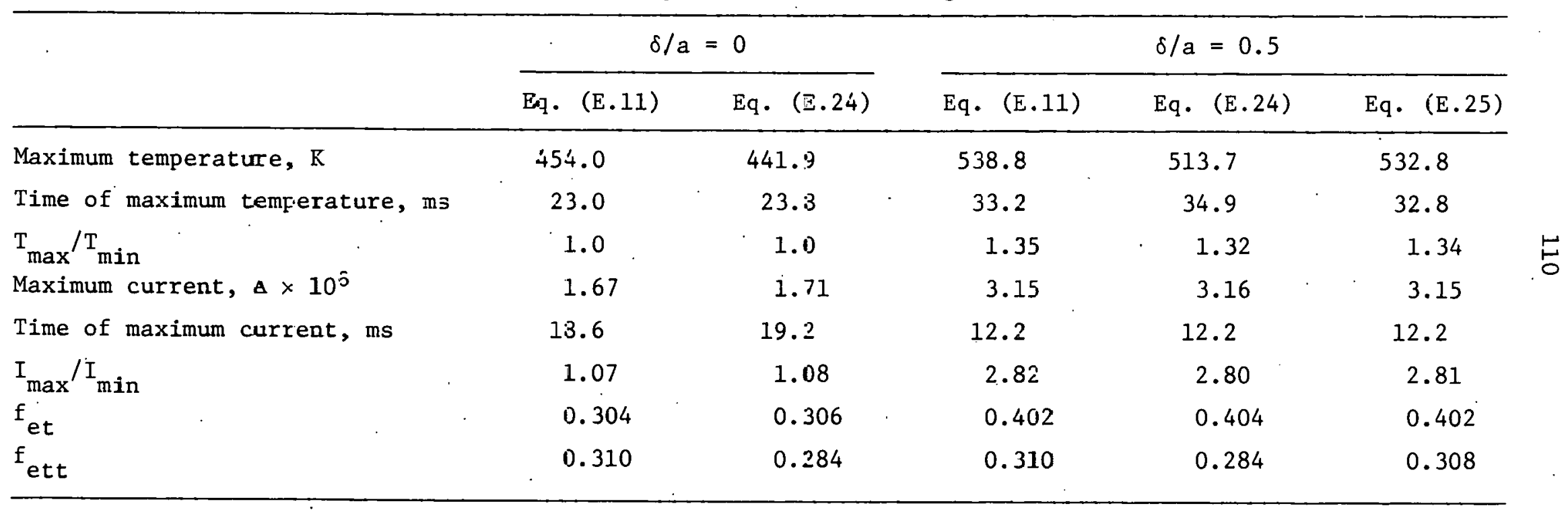


We assume that the internal heat generation resulting from Joule heating is spatially uniform in the radial direction. The time dependence of Joule heating is obtained from the lumped parameter model. The boundary conditions on the first wall (see Fig. E.4) are that at the front surface $(x=0)$, the expression

$\mathrm{q}_{\mathrm{T}}^{\prime \prime}(\mathrm{t})=-\left.\mathrm{k}(\mathrm{T}) \frac{\partial T(x, t)}{\partial \mathrm{x}}\right|_{\mathrm{x}=0}$

is satisfied and that at the back surface, the water coolant (at a bulk temperature of $20^{\circ} \mathrm{C}$ ) carries the heat away from the wall. The heat transfer coefficient is chosen as $6222 \mathrm{~W} / \mathrm{m}^{2} \cdot{ }^{\circ} \mathrm{C}$. The temperature distribution is obtained from the heat conduction equation,

$\frac{\partial}{\partial x}\left[k(T) \frac{\partial T(x, t)}{\partial x}\right]+q_{w E}^{\prime \prime \prime}(t)=C \rho_{p w} \frac{\partial T(x ; t)}{\partial t}$.

HEATING5 first obtains the steady-state distribution. The steady-state temperature profile is linear, with the front surface at $207^{\circ} \mathrm{C}$ and the rear surface at $123^{\circ} \mathrm{C}$ with $\delta / a=0$. The steady-state profile calculation includes the term ${ }^{11}$

$2.38 \times 13.1 \exp (-\mathrm{x} / 0.385) \mathrm{MW} / \mathrm{m}^{3}$,

where we assume that the neutron wall loading is $2.38 \mathrm{MW} / \mathrm{m}^{2}$.

The thermophysical properties of stainless steel vary with temperature. For $\tau_{D}=24 \mathrm{~ms}$ no phase change is necessary, but for $\tau_{D}=100 \mathrm{\mu s}$ the stainless steel not only melts but also vaporizes.

If melting occurs the boundary conditions between the liquid region $\ell$ and the solid region $s$ are

$-\left.k_{\ell} \frac{\partial \mathrm{T}_{\ell}}{\partial \mathrm{x}}\right|_{\mathrm{x}_{\ell s}}+\left.\mathrm{k}_{s} \frac{\partial \mathrm{T} s}{\partial s}\right|_{\mathrm{x}_{\ell s}}=\mathrm{h}_{s \ell} \rho \frac{\mathrm{dx} \ell s}{\mathrm{dt}}$, 
where $h_{s \ell}$ is the latent heat of fusion $(64 \mathrm{kcal} / \mathrm{g} \cdot \mathrm{K})$ and $\mathrm{x}_{\ell_{\mathrm{S}}}$ is the melting boundary, which moves as the heat is deposited.

Because HEATING5 uses a centered cylindrical geometry, a transformation of the surface heat flux rates and volumetric heat rates continually has to be made (see Fig. E.6). Figure E.6a shows the system with the plasma centered on the magnetic axis, and Fig. E.6b shows these same arcs in the vessel-centered system.

Figures E.10-12 indicate the results of HEATING5 for a centered plasma. For the 100-us case about 4 or $5 \mu \mathrm{m}$ of the wall ablates and about $40 \mu \mathrm{m}$ melts. For the $24-\mathrm{ms}$ case the wall neither melts nor ablates. In fact, the difference between the maximum and the average wall temperature is about $450^{\circ} \mathrm{C}$. Thermal fatigue estimates for the stainless steel have been made; it was found that about 350 aborts could be tolerated. The maximum wall temperature for the $100-\mu$ s case is $45400^{\circ} \mathrm{C}$, achieved at the surface at $70 \mu \mathrm{s}$. The maximum depth of wall melting is $35 \mu \mathrm{m}$, if the latent heat of fusion is included in the calculation, and occurs $100 \mu \mathrm{s}$ after the plasma disruption begins. If the latent heat of fusion is ignored in the calculation, $40 \mu \mathrm{m}$ of the wall melts. Because the heat of vaporization cannot be conveniently included in the HEATING5 code, the 100-us runs are correct up to $3090 \mathrm{~K}$ but not beyond that temperature.

One other fact to be noted is that if $\delta / a=0$, the internal heat generation rate due to eddy currents changes the wall temperatures by roughly $1 \%$ of the temperature change due to the surface heat flux. This can also be observed from the lumped parameter models. Therefore, for the circular wall, plasma-centered case; the temperature distribution is not sensitive to the eddy current heat generation rate.

The wall temperature resulting from a disruption of the nff-rentered plasma was also considered. Only the 24-ms disruption time was considered here. In no case did melting of the wall occur. Table E.6 is a summary of temperature data. Trends obtained from the lumped parameter model, which relate temperature to $\delta / a$ and time, are also apparent in the distributed parameter model. The temperature becomes uniform with $\phi$ at about a half thickness of the wall. Thus, a majority of thermal stress is concentrated in the first few millimeters of the wall. The effect of Joule heating becomes more pronounced in arc 1 as $\delta / a \rightarrow 1$. In this case the primary source of heat is the eddy currents. 


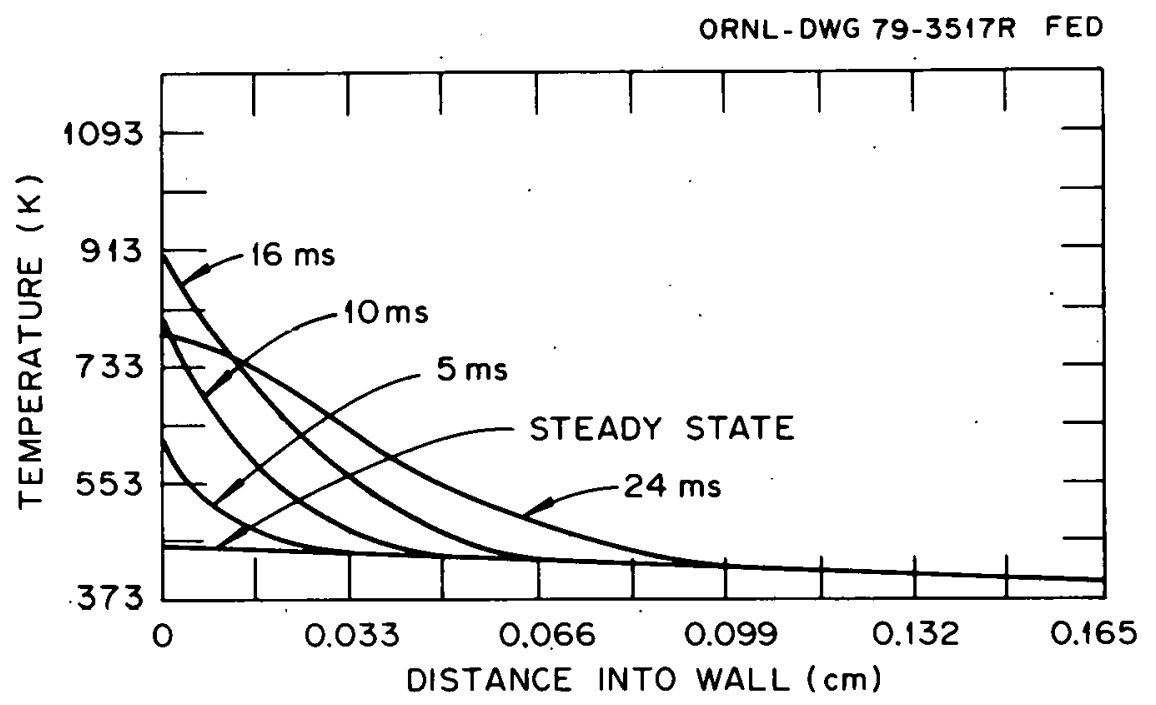

Fig. E.10. Temperature profile in wall for $\tau_{D}=24 \mathrm{~ms}$, constant velocity surface heat flux, including Joule heating. 


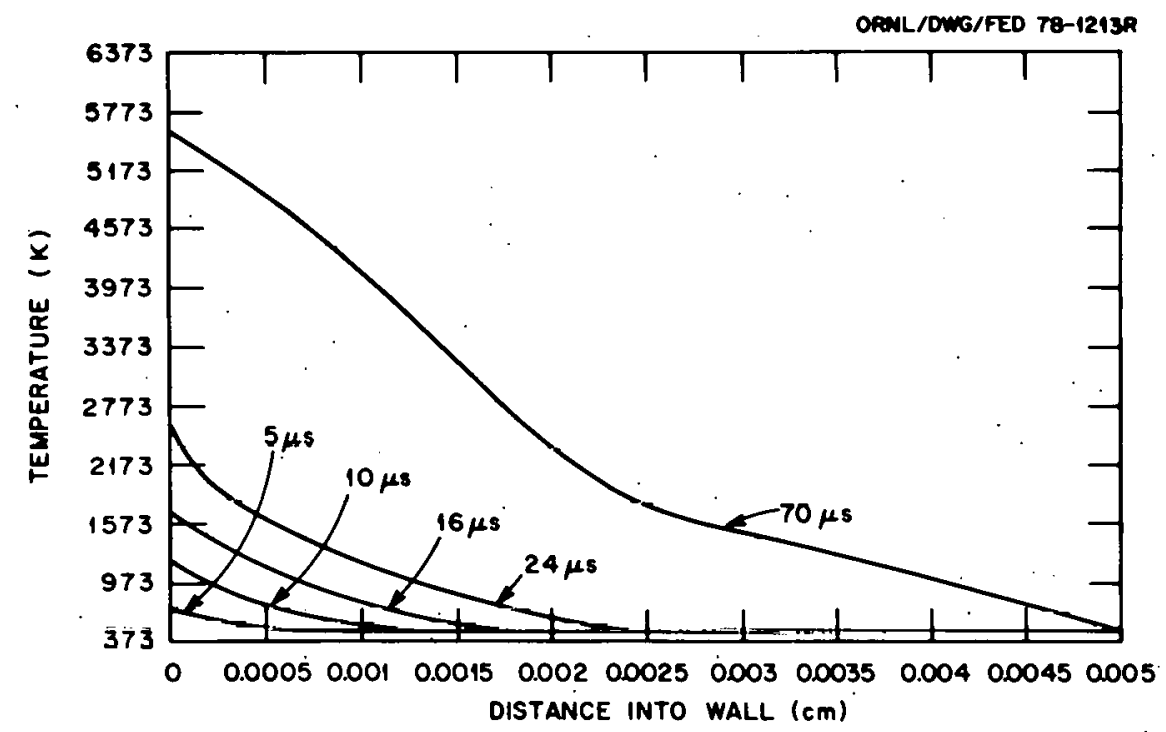

Fig. E.11. Wall temperature profile for $\tau_{D}=100 \mu \mathrm{s}$, constant velocity surface heat flux. 


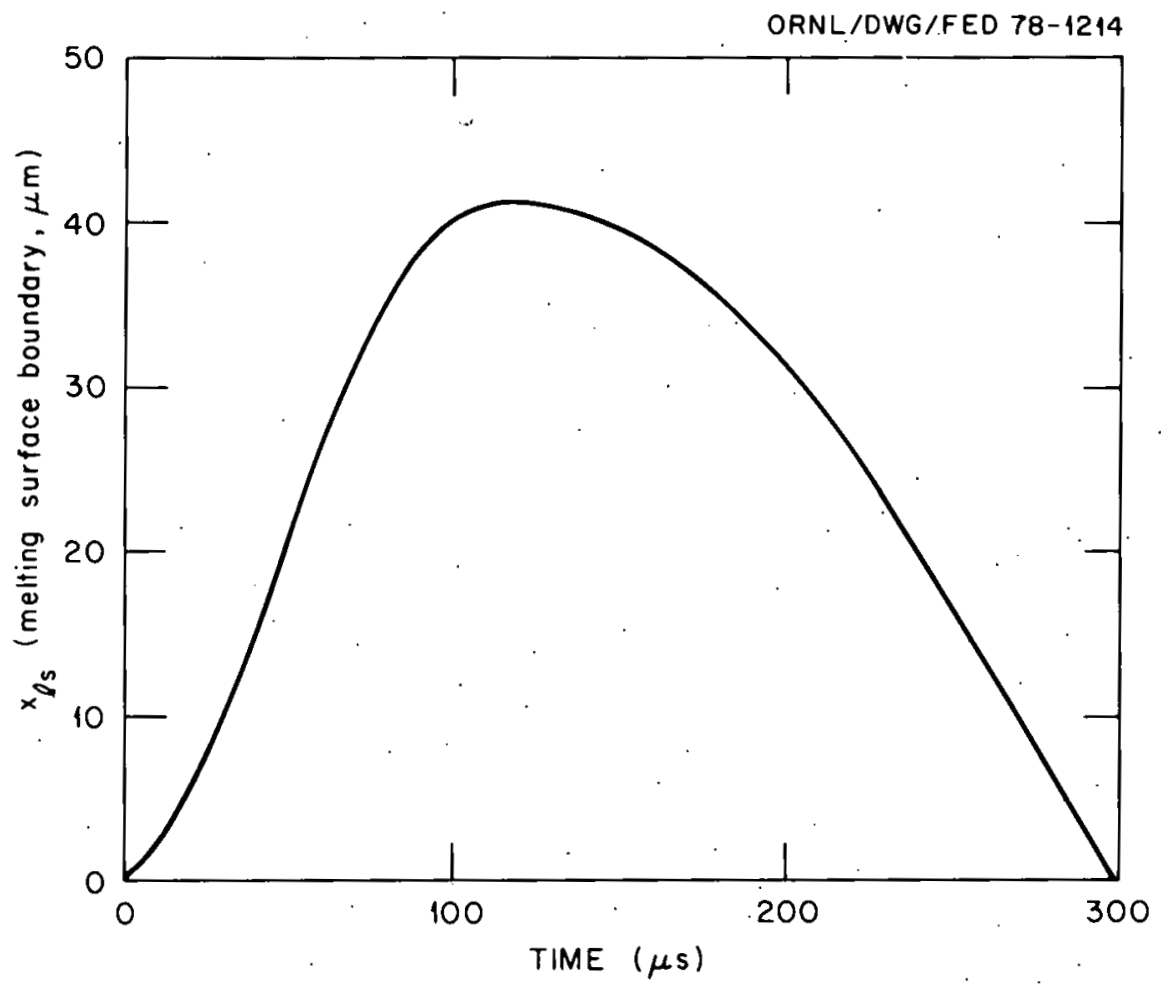

Fig. E.12. Solid liquid interface as a function of time. $\tau_{D}=100 \mu \mathrm{s}$, constant velocity surface heat flux. 
Table E.6. Results of the distributed parameter model

\begin{tabular}{|c|c|c|c|c|c|c|c|c|}
\hline$\delta / a$ & $\begin{array}{c}\mathrm{T}_{\max } \\
\left({ }^{\circ} \mathrm{C}\right)\end{array}$ & $\left({ }^{\circ} \mathrm{C}\right)$ & $\begin{array}{c}\text { Time of } \mathrm{T}_{\max } \\
\text { (ms) }\end{array}$ & $\begin{array}{l}\tau_{\text {eff }} \\
\text { (ms) }\end{array}$ & $\begin{array}{l}\Delta \mathrm{T} \\
\left({ }^{\circ} \mathrm{C}\right)\end{array}$ & $\begin{array}{c}\alpha^{a} \\
\left(10^{-\frac{5}{5}} \mathrm{~K}^{-1}\right)\end{array}$ & $\begin{array}{c}\mathrm{Sa}^{\bar{D}} \\
(\mathrm{ks} \dot{-})\end{array}$ & $\begin{array}{c}\text { Maximum number of MPD's } \\
\text { before wall failure }\end{array}$ \\
\hline \multicolumn{9}{|c|}{ Parabolic distribution [Eq. (E.I1)] } \\
\hline 0 & 719 & 256 & 18.6 & 24 & 463 & 1.932 & 166 & 350 \\
\hline 0.1 & 764 & 265 & 18.6 & 26.3 & 519 & 1.932 & 186 & 215 \\
\hline 0.3 & 896 & 298 & 22.2 & 30.7 & 598 & 1.944 & 2.5 & 135 \\
\hline 0.5 & 1015 & 334 & 25.5 & 35.1 & 681 & 1.946 & $24 \epsilon$ & 95 \\
\hline 0.7 & 1131 & 351 & 28.6 & 39.4 & 780 & 1.953 & 282 & 70 \\
\hline 0.977 & 1293 & 420 & 31.3 & $\angle 5.0$ & 873 & 1.971 & 319 & 48 \\
\hline \multicolumn{9}{|c|}{ Alternative distribution [Eq. (E.25)] } \\
\hline 0 & 632 & 236 & 19.3 & 24 & 396 & 1.93 & 142 & 450 \\
\hline 0.5 & 893 & 314 & 30.5 & 35.7 & 579 & 1.943 & 209 & 140 \\
\hline \multicolumn{9}{|c|}{ Cosine distribution [Eq. (E.26)] } \\
\hline 0.5 & 991 & 313 & 21.98 & 35.7 & 678 & 1.946 & 245 & 95 \\
\hline
\end{tabular}

$a_{\text {Thermal expansicn coefficient. }}$

$b_{\text {Stress amplitude. }}$ 
Figure E.13 illustrates the functional relationship between $\delta / a$ and the number of aborts. The number of MPD's to result in failure is calculated using a method outlined in the ASME Boiler Code.12 The maximum thermal stress $u_{\max }$ is given by

$\sigma_{\max }=\operatorname{Kn} \frac{E \alpha \Delta T}{1-a \mu}$

Here $\mathrm{K}$ is the stress concentration factor resulting from surface irregularities, $n$ depends on the number of constraints, a depends on the number of directions constrained, $\mu$ is Poisson's ratio, $\mathrm{E}$ is Young's modulus, $\Delta \mathrm{T}$ is the temperature difference in the wall resulting from the MPD, and $\alpha$ is the coefficient of expansion and is given by

$\alpha=1.7887 \times 10^{-5}+2.3977 \times 10^{-9} \mathrm{~T}+3.2692 \times 10^{-13} \mathrm{~T}^{2}$.

The units of $\alpha$ are ${ }^{\circ} \mathrm{C}^{-1}$; however, $\mathrm{K}$ is used in calculation. In this analysis $a, n$, and $K$ are taken to be $1 ; E$ is $26.10^{6} \mathrm{psi}$, as suggested by the ASME Boiler Code; Poisson's ratio is 0.3 ; and the stress amplitude is taken to be $(1 / 2) \sigma_{\max }$.

\section{E. 7 THE D-SHAPED $\cdot$ VESSEL}

Power-producing tokamaks will.most likely be of a noncircular cross section. A semiellipse or D lacks radial symmetry; therefore, the energy deposition in the first wall and other components will not be uniform during either normal operation or a MPD. Thus, as in the case of the offcentered plasma distribution function, a model must be developed to estimate the potential damage to the first wall. The particle and temperature distributions are still given by Eqs. (E.13) and (E.14), but now we take

$F(\rho, \theta)=\left[1-\left(\frac{\rho}{\rho_{D}}\right)^{2}\right]$, 


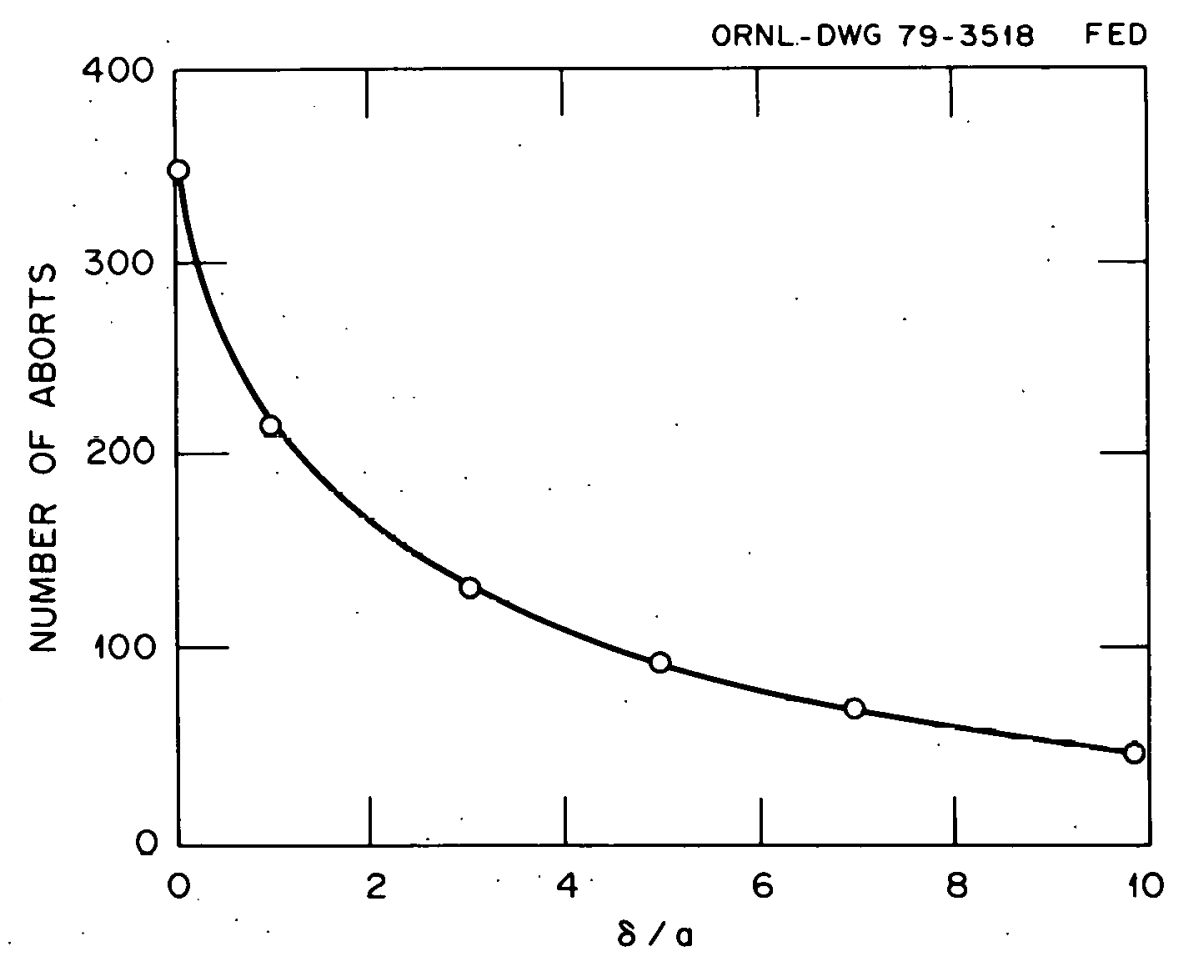

Fig. E.13. The max1mum number of MPD's before wall fallure for a clrcular wall Lukamak. 
where $\rho_{D}$ must now be computed for a semiellipse rather than a circle. The equation of an ellipse in polar coordinates is

$$
r_{D}^{2}=\frac{a^{2}}{1-\varepsilon^{2} \sin ^{2} \theta} \text {, }
$$

where $\varepsilon$ is the eccentricity and a, r, and $\theta$ are defined by Fig. E.14. Using the law of cosines the expression for $\rho_{D}(\theta)$ is

$\rho_{D}= \begin{cases}\sqrt{\delta_{D}^{2}+r_{D}^{2}-2 r_{D_{\delta}} \cos \theta} & \theta \leqslant 90^{\circ} \\ \sqrt{\delta_{D}^{2}+\left(-r_{D} \sin \theta\right)^{2}} & 90^{\circ}<\theta<180^{\circ}\end{cases}$

(for the purposes of analysis $\theta$ is extended from $\pi / 2$ to $\pi$ to obtain the straight-line portion of the $D$ ).

The total number of particles and energy in the system is conserved; thus, $\mathrm{n}_{\mathrm{o}}$ and $\mathrm{T}_{\mathrm{o}}$ are obtained using Eqs. (E.13) and (E.14). The total reactor volume available to the plasma will also be conserved; i.e.,

$$
\left(v_{p}\right)_{c}=\left(v_{p}\right)_{D}
$$

The volume of the plasma is approximately $2 \pi\left(R_{D}\right)$ (the cross-sectional area of the reactor). The cross-sectional area of the semiellipse is

$$
A_{c}=\frac{a^{2}}{2} \frac{\pi}{\sqrt{1-\varepsilon^{2}}}
$$

When the eccentricity is zero the area reduces to that of a semicircle. The major radius $R_{D}$ of the $D$-shaped reactor is thus 


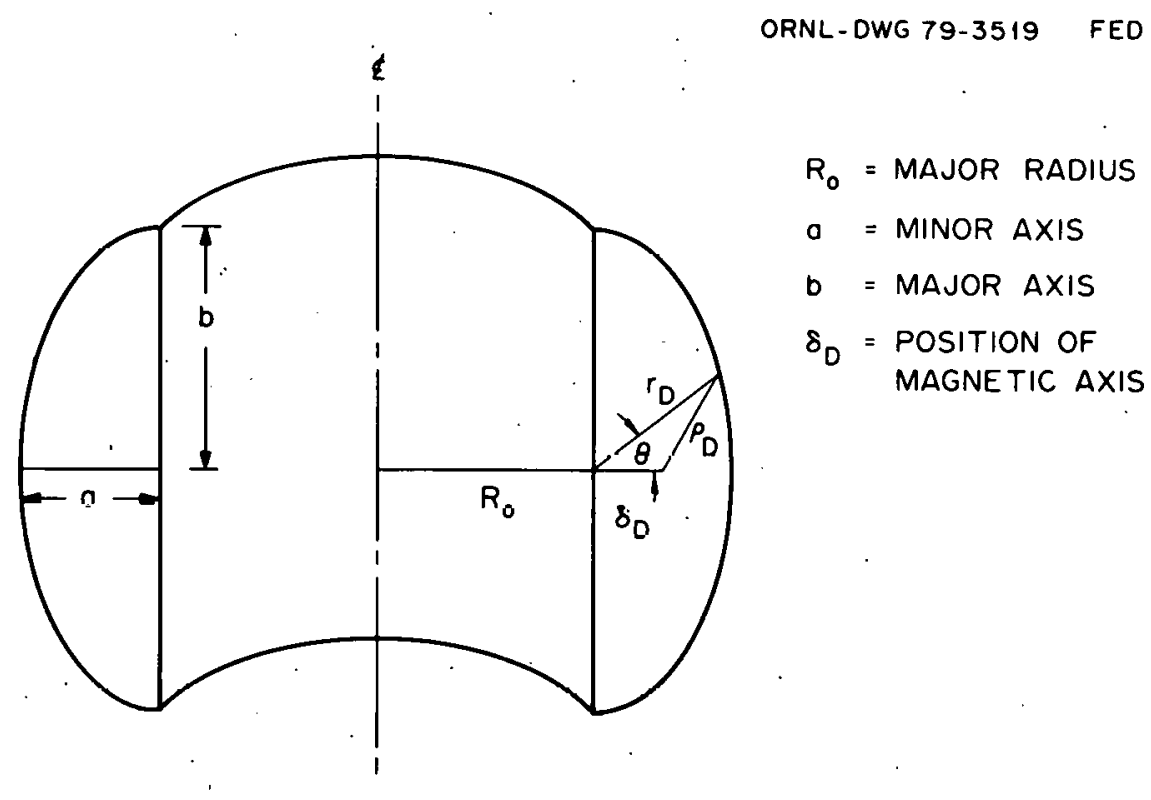

Fig. E.14. Geometric parameters associated with the D-shaped reactor. 


$$
R_{D}=\left(V_{p}\right)_{c} \frac{\sqrt{1-\varepsilon^{3}}}{\pi^{2} a^{2}} \text {. }
$$

To deduce the surface heat flux we again assume that the plasma particle moves radially outward at a constant velocity. Because the mutual inductance is more difficult to calculate in this case, some approximations were made in estimating $\mathrm{L}_{12}, \mathrm{~L}_{11}$, and $\mathrm{L}_{22}$.

Using arguments of symmetry only the upper half $(0-\pi)$ of the semiellipse need be considered. The five regions considered are three on the outer elliptical section and two on the inner flat plate section. A list of parameters defining the reactor cross sections studied is found in Table E.7. The magnetic center $\delta_{D}$ will be located at (1/6)a, (1/3)a, and $(2 / 3)$ a for all eccentricities.

Table E.7. Parameters describing reactor cross sections studied

\begin{tabular}{ccc}
\multicolumn{3}{c}{ cross sections studied } \\
\hline Eccentricity & $\mathrm{a}$ & $\mathrm{b}$ \\
\hline 0 & 1.770 & 1.770 \\
0.50 & 1.640 & 1.900 \\
0.968 & 0.886 & 3.540 \\
\hline
\end{tabular}

HEATING5 cannot be directly used for analysis of D-shaped reactors because it requires that all boundaries be parallel to the coordinate axis. A complete analysis of the first wall requires a separate run for each arc with a heat flux boundary condition

$\mathrm{q}=-\mathrm{k} \nabla \mathrm{T}$

on adjacent boundaries. The conduction heat flux must be obtained by successive approximations.

The effect that a MPD has on the first-wall lifetime is determined in the first several milliseconds. Because the maximum temperature is always achieved within the disruption time and because the time frame of significant poloidal conduction is greater than the disruption time, 
adiabatic boundaries between the arcs can be assumed without affecting the estimates of the wall's lifetime. The validity of this assumption can be checked by considering the thermal diffusivity $\mathrm{k} / \mathrm{\rho c}$.

A more straightforward check of this assumption can be made on the outer section of a reactor with zero eccentricity. In this case the coordinate axis coincides with the reactor boundaries. Arc 1 is analyzed by assuming an adiabatic boundary with arc 2 and also with conduction allowed between arcs 1 and 2 . The adiabatic assumption underpredicts the maximum temperature by $3.0^{\circ} \mathrm{C}$. The situation is just reversed for arc 3 , where the maximum wall temperature occurs, because heat will tend to flow out of it. Thus, this assumption, which is on the conservative side, can be expected to yield good results. Therefore, only the arc experiencing the maximum temperature rise in the lumped parameter model will be analyzed. When the eccentricity is zero polar coordinates are used. Cases with $\varepsilon>0$ are approximated by a planar slab with thickness $0.00165 \mathrm{~m}$ and length given by

$l= \begin{cases}\int_{\theta_{i}}^{\theta} \frac{a^{2}}{\sqrt{1-\varepsilon^{2} \sin ^{2}}} d \theta \quad & \theta \leqslant 90^{\circ} \\ b / 2 & 90^{\circ} \leqslant \theta \leqslant 180^{\circ}\end{cases}$

The arcs examined are between the angles (degrees) $0-30$ ( 1 ), 30-60 (2), 60-90 (3), 90-135 (4), and 135-180 (5). Each arc is identified by the number in parentheses. In general, the maximum temperature occurs in arc 3, the upper portion of the reactor. An exception is the case of $\varepsilon=0$ and $\delta_{D}=1.18$, where the maximum temperature vicuis in are 4 , the upper flat inner wall section; in this case, however, arc 3 shows the second-highest temperature rise. The current always peaks in arc 5 , the lower flat inner wall section, when the magnetic axis is located at $(1 / 6) a$ and $(1 / 3) a$. When the magnetic axis is located at $(2 / 3) a$, the peak current appears in the outer lower elliptical wall section, or 
arc 1. The higher temperatures always occur in arcs that have solid angles containing greater amounts of plasma. The current, on the other hand, always peaks in arcs that have wall segments closest to the magnetic center of the distribution.

Trends show that the maximum wall temperature increases as $\varepsilon \rightarrow 1$ for a fixed $\delta_{\mathrm{D}}$. The spread between the maximum and minimum wall temperature also increases with $\varepsilon$. The situation is reversed when $\varepsilon$ is held constant and the position of the magnetic axis is allowed to vary.

Plots of the distribution show that for fixed $\delta_{D}$ more and more plasma resides in the solid angle associated with arc 3 as $\varepsilon \rightarrow 1$; thus, more energy is incident on this wall segment. However, when the eccentricity is held constant and the position of the magnetic axis is allowed to vary, plasma is shifted into arcs 4 and 5 , thus resulting in a more even distribution of energy.

The fraction of magnetic energy stored in the plasma that is converted to heat in the wall, $f_{e t}$, and the fraction of magnetic and thermal energy that goes into heating the wall, $\mathrm{f}_{t t}$, are computed using Eqs. (E.22). and (E.23), respectively. (The values of $f_{\text {et }}$ are considered an overestimate.) These numbers can be found in Table E.8. The scheme

Table E.8. A summary of $f_{e t}$ and $f_{t t}$ for the $D$-shaped vessel

\begin{tabular}{llll}
\hline$\varepsilon$ & $\begin{array}{c}\delta_{\mathrm{D}} / \mathrm{a} \\
(\mathrm{m} / \mathrm{m})\end{array}$ & $\mathrm{f}_{\text {et }}$ & $\mathrm{f}_{\mathrm{tt}}$ \\
\hline 0 & $1 / 6$ & 0.972 & 0.404 \\
0 & $1 / 3$ & 0.962 & 0.346 \\
0 & $2 / 3$ & 0.940 & 0.312 \\
0.500 & $1 / 6$ & 0.982 & 0.390 \\
0.500 & $1 / 3$ & 0.966 & 0.354 \\
0.500 & $2 / 3$ & 0.976 & 0.318 \\
0.968 & $1 / 6$ & 1 & 0.474 \\
0.968 & $1 / 3$ & 1 & 0.452 \\
0.968 & $2 / 3$ & 1 & 0.420 \\
\hline
\end{tabular}


used to calculate electromagnetic properties either underestimates the total magnetic energy by using an incorrect self-inductance or overestimates the induced eddy currents by using a mutual inductance that is too large or both; however, the trend displayed is correct. The fraction of magnetic energy that is converted to heat in the wall increases with the eccentricity. As the eccentricity increases the mutual inductance in arcs 1 and 5 increases faster than it decreases between the plasma and the remaining arcs. Thus, greater eddy currents are induced, which, in turn, lead to increased Joule heating.

The fraction of magnetic and thermal energy that is converted to heat is also an overestimation because of the problem with $f_{\text {et }}$. However, the error is considered slight because the majority of the wall heating is from the surface heat $f l u x$. In general, $f_{t t}$ increases gradually for eccentricities from 0.0 to 0.5 and then rapidly as $\varepsilon \rightarrow 1$. When $\varepsilon$ is held constant and $\delta_{D}$ is allowed to vary, $f_{t t}$ decreases as $\delta_{n}$ increases.

Arcs with wall segments closest to the magnetic axis experience the greatest wall currents. Arcs that have associated solid angles containing the greatest amount of plasma experience the greatest temperature increases.

Trends discussed earlier for the circular wall are also apparent in the HEATING5 runs for the $D$. The disruption time is assumed to be $24 \mathrm{~ms}$. The maximum temperature in the wall increases, for a given $\delta_{D}$, as $\varepsilon \rightarrow 1$. With the eccentricity held constant the maximum temperature decreases as $\delta_{D}$ increases. Significant results from the HEATING5 output are listed in Table E.9.

\section{E. 8 GONCIUSTONS}

'l'he tollowing conclusions àre derived from the analysis.

(1) It is likely that the plasma disruption time $\tau_{D}$ will be longer than previously anticipated. Instead of $100 \mu \mathrm{s}$ it is probable that the disruption time will be of the order of milliseconds for the ETF.

(2) The eddy currents are much more important in first-wall stress considerations and power supply protection than in contributing to 
Table E.9. Results of the distributed parameter model for the D-shaped wall

\begin{tabular}{|c|c|c|c|c|c|c|c|c|c|c|}
\hline$\varepsilon$ & $\begin{array}{c}\delta_{D} / \epsilon \\
(m / m)\end{array}$ & ${\stackrel{\mathrm{T}}{\mathrm{T}_{\mathrm{max}}}}_{\left({ }^{\circ} \mathrm{C}\right)}$ & $\begin{array}{c}\text { Time } \mathrm{T}_{\max } \\
\text { occurrec } \\
\text { (ms) }\end{array}$ & $\begin{array}{c}\tau_{\text {eff }} \\
(\mathrm{ms})\end{array}$ & $\begin{array}{l}\overline{\mathrm{T}} \\
\left({ }^{\circ} \mathrm{C}\right)\end{array}$ & $\stackrel{\Delta \mathrm{T}}{\left({ }^{\circ} \mathrm{C}\right)}$ & $\begin{array}{c}\alpha^{a} \\
\left(\times 10^{-5}\right. \\
\left.{ }^{\circ} \mathrm{C}^{-1}\right)\end{array}$ & $\begin{array}{c}\mathrm{Sa}^{b} \\
(\mathrm{ksi})\end{array}$ & $\begin{array}{l}\text { Maximum number of } \\
\text { MPD's before failure }\end{array}$ & $\begin{array}{c}\alpha^{c} \\
\left(\times 10^{-6}\right. \\
\left.\mathrm{m}^{2} / \mathrm{s}\right)\end{array}$ \\
\hline 0 & $1 / 6$ & 675 & 23.4 & 33.0 & 254 & 421 & 1.92 & 150 & 360 & 4.20 \\
\hline 0 & $1 / 3$ & 607 & 23.7 & 32.9 & 242 & 365 & 1.92 & 130 & 580 & 4.17 \\
\hline 0 & $2 / 3$ & 536 & 19.5 & 28.3 & 223 & 313 & 1.91 & 111 & 910 & 4.17 \\
\hline 0.500 & $1 / 6$ & 590 & 23.4 & 35.0 & 256 & 434 & 1.92 & 154 & 340 & 4.20 \\
\hline 0.500 & $1 / 3$ & 560 & 23.6 & 34.8 & 250 & 410 & 1.92 & 146 & 460 & 4.20 \\
\hline 0.500 & $2 / 3$ & 543 & 24.2 & 36.6 & 227 & 317 & 1.91 & 112 & 900 & 4.17 \\
\hline 0.968 & $1 / 6$ & 1188 & 32.2 & 47.3 & 393 & 795 & 1.96 & 290 & 60 & 5.02 \\
\hline 0.968 & $1 / 3$ & 1149 & 32.4 & 46.8 & 385 & .764 & 1.96 & 278 & 66 & 4.98 \\
\hline 0.968 & $2 / 3$ & $\therefore 045$ & 33.0 & 46.4 & 362 & 683 & 1.95 & 248 & 90 & 4.53 \\
\hline
\end{tabular}

$\alpha_{\text {Thermal expansion coefficient. }}$

$b_{\text {Stress amplitude. }}$

"Thermal diffusivity. 
the heat load through $I^{2} R$ Joule heating. It seems that in most cases except for extremely asymmetric configurations, $<10 \%$ of the temperature change in the wall will be due to the induced eddy currents.

(3) The peak temperature is not very sensitive to the shape of the heat pulse that strikes the wall. It is primarily due to the disruption time $\tau_{D}$ and the total thermal energy in the plasma, which determine the temperature distribution in the wall.

(4) The maximum wall temperature for circular tokamaks (about $1000^{\circ} \mathrm{C}$ for $\delta / a=0.5)$ increases as $\delta / a \rightarrow 1$, and the maximum temperature is always in the inside region of the torus. For D-shaped tokamaks the maximum wa11. temperature increases as the eccentricity $\varepsilon \rightarrow 1$ and decreases as $\delta_{D} / a \rightarrow 1$. The maximum temperature (about $1100^{\circ} \mathrm{C}$ for $\varepsilon=0.968$ and $\left.\delta_{D} / a=1 / 6\right)$ occurs in the top portion of the $D$, a result depending on the constant radial velocity assumption.

(5) The maximum number of MPD's before wall failure decreases as $\delta / a \rightarrow 1$ with 48 being the minimum number for $\tau_{D}=24 \mathrm{~ms}$ and an off-centered circular plasma for the ETF. Because of more surface area per plasma volume in the D-shaped tokamak, this number increases to 60 MPD's for the worst case.

\section{REFERENCES}

1. R. Behrisch, Nuc1. Fusion 12, 695 (1972).

2. T. Kammash, Fusion Reactor Physics, Principles and Technology, Ann Arbor Science Publishers, Inc., Ann Arbor, Michigan, 1977.

3. D. L. Smith and I. Charak, Proc. 7th Symp. on Engineering Problems of Fusion Reserarah, Vol. II, p. 1465 (1978).

4. R. B. White, D. A. Monticello, and M. N. Rusenbluth, Phys. Rev. Lett. 39, 1618 (1977).

5. J. A. Holmes et a1., ORNL/TM-6707, Oak Ridge, Tennessee (1979).

6. J. D. Callen et al., ORNL/TM-6564, Oak Ridge, Tennessee (1978).

7. N. R. Sauthoff et al., Nucl. Fusion 18, 1445 (1978).

8. B. Carreras, B. V. Wadde11, and H. R. Hicks, ORNL/TM-6175, Oak Ridge, Tennessee (1978).

9. C. S. Kim, ANL-75-55, Argonne National Laboratory, Argonne, Illinois (1975). 
10. W. D. Turner et al., ORNL CSD/TM-15, Oak Ridge, Tennessee (1977).

11. R. W. Werner, ORNL/TM-5964, Oak Ridge, Tennessee (1977).

12. ASME Boiler and Pressure Vessel Code, Section III-Division 1. Nuclear Power Plant Components, Subsections NA: General Requirements and NB: Class 1 Components, American Society of Mechanical Engineers, New York (1974). 
THIS PAGE

\section{WAS INTENTIONALLY LEFT BLANK}


ORNL/TM-7153

Dist. Category UC-20 d

\section{INTERNAL DISTRIBUTION}

1. T. G. Brown

2. E. H. Bryant

3. J. L. Dunlap

4. G. E. Gorker

5. P. N. Haubenreich

6. W. A. Houlberg

7. T. C. Jernigan

8. J. Kirchner

9. D. H. Metzler

10. T. V. Miskel1

11. J. G. Murray

12. W. D. Nelson

13. R. E. Nygren

14. J. Sheffield

15. G. E. Smith
16. P. T. Spampinato

17-21. D. Steiner

22. R. S. Stone

23. G. W. Wiseman

24. F. Wu

25. T. G. Yow

26-27. Laboratory Records Department

28-29. Central Research Library

30. Document Reference Section

31. Laboratory Records, ORNL-RC

32. ORNL Patent Office

33-34. Fusion Energy Division

Library

35. Fusion Energy Division

Reports Office

\section{EXTERNAL DISTRIBUTION}

36. M. A. Abdou, Associate Director, Fusion Power Program, B1dg. 207-C101, Argonne National Laboratory, 9700 South Cass Ave., Argonne, IL 60439

37. N. A. Amherd, Fusion Power Program, Advanced Systems Department, Electric Power Research Institute, 3412 Hillview Ave., P.0. Box 10412, Palo Alto, CA 94304

38. J. L. Anderson, Los Alamos Scientific Laboratory, P.0. Box 1663, CMB-3, Mail Stop 348, Los Alamos, NM 87545

39. D. J. Anthony, Manager, Advanced Energy Systems, Energy Systems Programs Department, Bldg. 23, Rm. 290, General Electric Co., Schenectady, NY 12345

40. R. E. Aronstein, Bechtel National, Inc., P.0. Box 3965, San Francicco, CA 94119

41. M. P. Bachynski, MPB Technologies, Inc., P.0. Box 160, 21051 North Service Road/Trans-Canada Highway, Ste-Anne-de-Bellevue, Quebec, H9X 3L5, Canada

42. C. C. Baker, Fusion Power Program, Bldg. 208, Argonne National Laboratory, 9700 South Cass Ave., Argonne, IL 60439

43. R. E. Balzhiser, Director, Fossil Fuel and Advanced Systems, Electric Power Research Institute, P.0. Box 10412, Palo Alto, CA 94304

44. G. C. Banick, Cencral. Electric Co., 253 Main East, Oak Ridge, TN 37830

45. T. H. Batzer, Lawrence Livermore Laboratory, P.0. Box 808, L-536, Livermore, CA 94550

46. J. E. Baublitz, Office of Fusion Energy, ETM, Department of Energy, Mail Stop G-234, Washington, DC 20545 
47. W. Bauer, Physical Research Division, Sandia LaboratoriesLivermore, Livermore, CA 94550

48. J. Beal, General Atomic Co., P.O. Box 81608, San Diego, CA 92138

49. D. S. Beard, Office of Fusion Energy, ETM, Department of Energy, Mail Stop G-234, Washington, DC 20545

50. W. R. Becraft, M/C 150, General Electric Co., 175 Curtner Ave., San Jose, CA 95125

51. R. J. Beeley, ETEC, Rockwell International, P.0. Box 1449, Canoga Park, CA 91304

52. G. W. Benedict, U.S. Department of Energy, Oak Ridge Operations, P.O. Box E, Oak Ridge, TN 37830

53. F. Bennett, Princeton Plasma Physics Laboratory, Princeton University, P.0. Box 451, Princeton, NJ 08540

54. D. C. Berkey, Vice President and General Manager, Energy System and Technology Division, General Electric Co., P.0. Box 7600, Stamford, CT 06904

55. E. S. Bettis, Science Applications, Inc., 800 Oak Ridge Turnpike, Oak Ridge, TN 37830

56. R. F. Beuligmann, Program D1rector, Energy Systems, General Dynamics, Convair Division, P.0. Box 80847, Mail Zone 12-1070, San Diego, CA 92138

57. S. L. Bogart, Science Applications, Inc., 8400 Westpark Drive, McLean, VA 22102

58. R. Botwin, C47-05, Grumman Aerospace Corp., Bethpage, NY 11714

59. N. Bowen, Princeton Plasma Physics Laboratory, Princeton University, P.0. Box 451, Princeton, NJ 08540

60. W. B. Briggs, McDonnell-Douglas Astronautics Co., P.0. Box 516, St. Louis, MO 63166

61. G. Bronner, Princeton Plasma Physics Laboratory, Princeton University, P.0. Box 451, Princeton, NJ 08540

62. S. C. Burnett, General Atomic Co., P.0. Box 81608, San Diego, CA 92138

63. C. S. Caldwell, Babcock and Wilcox, Lynchburg Research Center, P.0. Box 1260, Lynchburg, VA 24505

64. J. D. Callen, Department of Nuclear Engineering, University of Wisconsin, Madison, WI 53706

65. A. T. Christensen, General Electric Co., Center for Energy Systems, 777 Fourteenth St., Washington, DC 20006

66. J. F. Clarke, Deputy Director, Office of Fusion Energy, ETM, Department of Energy, Mail Stop G-234, Washington, DC 20545

67. R. G. Clemmer, Fusion Power Program, Argonne National Laboratory, 9700 South Cass Ave., Argonne, IL 60439

68. F. E. Coffman, Office of Fusion Energy, ETM, Department of Energy, Mail Stúp G-234, Washington, DC . 20545

69. D. R. Cohn, MIT Plasma Fusion Center, NW 16-206, 167 Albany Street, Cambridge, MA 02139

70. R. W. Conn, Department of Chemical, Nuclear, and Thermal Engineering, Boelter Hall, University of California, Los Angeles, CA. 90024

71. J. W. Coursen, C36-05, Grumman Aerospace Corp., Bethpage, NY 11714

72. J. G. Crocker, EG\&G Idaho, Inc., P.0. Box 1625, Idaho Falls, ID 83401 
73. C. C. Damm, Lawrence Livermore Laboratory, P.0. Box 808, L-441, Livermore, CA 94550

74. R. A. Dandl, 1122 Calle de los Serranos, San Marcos, CA 92069

75. M. H. Dandridge, Grumman Aerospace Corp., Power Plant and Environment, B-14-35, Bethpage, NY 11714

76. R. Daniels, Princeton Plasma Physics Laboratory, Princeton University, P.0. Box 451, Princeton, NJ 08540

77. R. C. Davidson, MIT Plasma Fusion Center, 77 Massachusetts Ave., Cambridge, MA 02139

78. N. A. Davies, Office of Fusion Energy, ETM, Department of Energy, Mail Stop G-234, Washington, DC 20545

79. J. W. Davis, McDonnell-Douglas Astronautics Co., East E457, B1dg. 81/1/C7, P.0. Box 516, St. Louis, MO 63166

80. M. J. Davis, Sandia Laboratories-Livermore, Dept. 5830, Livermore, CA 94550

81. S. 0. Dean, Director,.Fusion Energy Development, Science Applications, Inc., 2 Professional Drive, Suite 249, Gaithersburg, MD 20760

82. J. F. Decker, Office of Fusion Energy, ETM, Department of Energy, Mail Stop G-234, Washington, DC 20545

83. D. DeFreece, McDonne11-Douglas Astronautics Co., Dept. E451, Bldg. 81/1/C7, P.0. Box 516, St. Louis, MO 63166

84. A. Deitz, Princeton Plasma Physics Laboratory, Princeton University, P.0. Box 451, Princeton, NJ 08540

85. D. A. Dingee, Program Manager, Pacific Northwest Laboratories, Fusion Technology, Battelle Blvd., Richland, WA 99352

86. J. N. Doggett, Lawrence Livermore Laboratory, P.0. Box 808, L-441, Livermore, CA 94550

87. H. Dreicer, Division Leader, Los Alamos Scientific Laboratory, CRT, P.O. Box 1663, Los Alamos, NM 87545

88. R. J. Dugal, Energy Systems-Power Division, Ralph M. Parsons Co., 100 West Walnut St., Pasadena, CA 91124

89. W. R. Ellis, Office of Fusion Energy, ETM, Department of Energy, Mail Stop G-234, Washington, DC 20545

90. G. A. Emmert, Department of Nuclear Engineering, University of Wisconsin, Madison, WI 53706

91. B. Engholm, General Atomic Co., P.0. Box 81608, San Diego, CA 92138

92. H. P. Eubank, Princeton Plasma Physics Laboratory, Princeton University, P.0. Box 451, Princeton, NJ 08540

93. E. Evans, Vice President, Westinghouse-Hanford, P.0. Box 1970, Richland, WA 99352

94. F. Farfaletti-Casali, Engineering Division, Joint Research Center, Ispra Establishment, 21020 Ispra (Varese), Italy

95. B. J. Fedor, EBASCO Services, Inc., Princeton Plasma Physics Laboratory, Princeton University, P.O. Box 451, Princeton, NJ 08540

96. J. J. Ferrante, Manager, Large Superconducting Program, Bldg. 36-241, General Electric Co., 1 River Rd., Schenectady, NY 12345

97. D. Field, General Atomic Co., P.0. Box 81608, San Diego, CA 92138

98. J. File, Princeton Plasma Physics Laboratory, Princeton University, P.0. Box 451, Princeton, NJ 08540 
99. H. K. Forsen, Exxon Nuclear Co., Inc., 777 106th Ave., NE, Bellevue, WA 98004

100. J. Foster, Jr., TRW, Inc., 1 Space Park, Bldg. R4-2004, Redondo Beach, CA 90278

101. T. K. Fowler, Associate Director for MFE, Lawrence Livermore Laboratory, P.0. Box 808, L-436, Livermore, CA 94550

102. R. L. Freeman, General Atomic Co., P.0. Box 81608, San Diego, CA 92138

103. J. W. French, EBASCO Services, Inc., Princeton Plasma Physics Laboratory, Princeton Univiversity, Forrestal Campus, CN-59, Princeton, NJ 08540

104. H. P. Furth, Princeton Plasma Physics Laboratory, Princeton University, P.0. Box 451, Princeton, NJ 08540

105. E. P. Gagnon, United Technologies Research Center, Silver Lane, Mail Stop 44, East Hartford, CT 06108

106. J. G. Gavlin, J1, Presldenl, Grumman Aesuspace Curp., A01=111, Bethpage, NY 11714

107. J. Gilleland, Manager, Fusion Projects, General Atomic Co., P.O. Box 81608, San Diego, CA 92138

108. R. E. Gold, Westinghouse Electric Corp., Fusion Power Systems Department, P.0. Box 10864, Pittsburgh, PA 15236

109. S. Goldfarb, Princeton Plasma Physics Laboratory, Princeton University, P.0. Box 451, Princeton, NJ 08540

110. J. Golzy, c/o G. V. S. Raju, Department of Electrical Engineering, Ohio University, Athens, OH 45701

111. M. B. Gottlieb, Director, Princeton Plasma Physics Laboratory, Princeton University, P.0. Box 451, Princeton, NJ 08540

112. R.'W. Could, Bldg. 116-81, California Institute of Technology, Pasadena, CA 91109

113. D. W. Graumann, Genera1 Atomic Co., P.0. Box 81608, San Diego, CA 92138

114. G. E. Guest, Plasma Theory Department, General Atomic Co., P.o. Box 81608, San Dicgo, CA 92138

115. E. R. Hager, General Atomic Co., P.0. Box 81608, Mail Stop L-549, San Dlego, CA 92138

116. S. D. Harkness, Manager, Fuel Development Materials Technology, Bettis Atomic Power Laboratory, P.0. Box 79, West Mifflin, PA 15122

117. R. J. Hawryluk, Princeton Plasma Physics Laboratory, Princeton University, P.0. Box 451, Princeton, NJ 08540

118. C. R. Head, Office of Fusion Energy, ETM, Department of Energy, Mail Stop G-234, Washington, DC 20545

119. H. K. Hebeler, President, Boeing Engineering and Construction Co., P.0. Box 3707 , Seattle, WA 98124

120. R. L. Hirsch, General Manager fon Exploralory Research, Exxun Research and Engineering Co., Box 101, Florham Park, NJ 07932

121. N. J. Hoffman, ETEC, P.O. Box 1449, T 486, Canoga Park, CA 91304

122. J. J. Holmes, Westinghouse-Hanford Engineering Development Laboratory, P.0. Box 1970, Richland, WA 99352

123. W. G. Homeyer, General Atomic Co., P.0. Box 81608, San D1ego, CA 92138

124. W. M. Hooke, Princeton Plasma Physics Laboratory, Princeton University, P.0. Box 451, Princeton, NJ 08540 
125. J. C. Hosea, Princeton Plasma Physics Laboratory, Princeton University, P.O. Box 451, Princeton, NJ 08540

126. R. Huse, Manager, Research and Development Department, PSE\&G Research Corp., 80 Park Place, Newark, NJ 07101

127. D. L. Jassby, Princeton Plasma Physics Laboratory, Princeton University, P.0. Box 451, Princeton, NJ 08540

128. B. K. Jensen, PSE\&G Research Corp., 80 Park Place, Rm. 1116, Newark, NJ 07101

129. N. E. Johnson, Science Applications, Inc., 800 Oak Ridge Turnpike, Oak Ridge, TN 37830

130. J. B. Joyce, Princeton Plasma Physics Laboratory, Princeton University, P.O. Box 451, Princeton, NJ 08540

131. E. E. Kintner, Director, Office of Fusion Energy, ETM, Department of Energy, Mail Stop G-234, Washington, DC 20545

132. R. A. Krakowski, Los Alamos Scientific Laboratory, P.0. Box 1663, CTR-12, Mail Stop 641, Los Alamos, NM 87545

133. N. A. Krall, JAYCOR, 1401 Camino Del Mar, Del Mar, CA 92014

134. G. L. Kulcinski, University of Wisconsin, Department of Nuclear Engineering, Engineering Research B1dg., Rm. 439, 1500 Johnson Drive, Madison, WI 53706

135. C. A. Kukielka, Science Applications, Inc., 800 Oak Ridge Turnpike, Oak Ridge, TN 37830

136. D. L. Kummer, McDonne11-Douglas Astronautics Co., Dept. E457, Bldg. 81/132, P.0. Box 516, St. Louis, MO 63166

137. J. Landis, Senior Vice President, Stone and Webster Engineering Corp., 245 Summer St., Boston, MA 02107

138. T. S. Latham, United Technologies Research Center, Silver Lane, Mail Stop 44, East Hartford, CT 06108

139. J. Lawson, Princeton Plasma Physics Laboratory, Princeton University, P.0. Box 451, Princeton, NJ 08540

140. L. M. Lidsky, MIT Plasma Fusion Center, Cambridge, MA 02139

141. E. F. Lowc11, General Manager, Energy Systems Programs Department, Bldg. 2-455, General Electric Co., 1 River Rd., Schenectady, NY 12345

142. D. G. McAlees, Manager, Program Development, Laser Enrichment Department, Exxon Nuclear Co., Inc., 777 106th Ave., NE, Bellevuc, WA 98004

143. D. J. McFarlin, United Technologies Research Cenlei, Silver Lane, Mail Stop 44, East Hartford, CT 06108

144. V. A. Maroni, Argonne National Laboratory, CEN/205, 9700 South Cass Ave., Argonne, IL 60439

145. W. Marton, Office of Fusion Energy, ETM, Department of Energy, Mail Stop G-234, Washington, DC 20545

146. L. Masson, EC\&S Idaho, Idaho National Engineering Laboratory, P.0. Box 1625, Idaho Falls, ID 83401

147. D. M. Meade, Princeton Plasma Physics Laboratory, Princeton University, P.0. Box 451, Princeton, NJ 08540

148. A.. T. Mense, Subcommittee on Energy Research and Production, B-374, Rayburn House Office Bldg., Washington, DC 20515

149. L. Michaels, Princeton Plasma Physics Laboratory, Princeton University, P.O. Box 451, Princeton, NJ 08540

150. R. Micich, Grumman Aerospace Corp., Bethpage, NY 11714 
151. R. L. Miller, General Atomic Co., P.0. Bxo 81608, San Diego, CA 92138

152. R. G. Mills, Princeton Plasma Physics Laboratory, Princeton University, P.0. Box 451, Princeton, NJ 08540

153. J. T. D. Mitche11, Culham Laboratory, Abingdon, Oxon, OX14 3DB, United Kingdom

154. P. B. Mohr, Lawrence Livermore Laboratory, P.0. Box 808, Livermore, CA 94550

155. R. W. Moir, Lawrence Livermore Laboratory, P.0. Box 808, Livermore, CA 94550

156. D. B. Montgomery, MIT Plasma Fusion Center, 170 Albany St., Cambridge, MA 02139

157. K. Moses, TRW, Inc., R-1/1078, 1 Space Park, Redondo Beach, CA 90278

158. R. E. Muller, Aerojet Manufacturing $\mathrm{Cn}$, , 601 South Flacenc1à Áve., T.O. Bux 421U, Fullerton, CA 92934

159. A. E. Munier, Grumman Aerospace Corp., Bethpage, NY 11714

160. M. R. Murphy, Office of Fusion Energy, ETM, Department of Energy, Mail Stop G-234, Washington, DC 20545

161. T. Ohkawa, Vice President, General Atomic Co., P.0. Box 81608, San Diego, CA 92138

162. M. Okabayashi, Princeton Plasma Physics Laboratory, Princeton University, P.0. Box 451, Princeton, NJ 08540

163. R. J. Onega, Department of Mechanical Engineering, Virginia Polytechnic Institute and State University, Blacksburg, VA 24061

164. R. R. Parker, MIT, NW 14-2506, National Magnet Laboratory, 170 Albany St., Cambridge, MA 02139

165. P. B. Parks, General Atomic Co., P.0. Box 81608, San Diego, CA 92138

166. B. Yease; Culham Laboratory, Abingdon, Oxon, OX14 3DB, United Kingdom

167. M. Yorkolab, Massachusetts Institute of Technolugy, Cambridge, MA 02139

168. T., K. Price, U.S. DeparLmene of Energy, Oak Ridge Operations, P.O. Box E, Oak Ridge, TN 37830

169. R. E. Price, Office of Fusion Energy, ETM, Department of Energy, Mail Stop G-234, Washington; DC 20545

170. D. H. Priester, Office of Fusion Energy, ETM, Department of Energy, Mail Stop G-234, Washington, DC 20545

171. F. A. Puhn, General Atomic Cn., P.0. Box 81608, San Diego, CA 92138

172. R. V. Pyle, University of California, Lawrence Berkeley Laboratory, Berkeley, CA 94720

173. G. V: S. Raju, Department of Electrical Engineering, Ohin Univeraity, Athens, OH 45701

17.4. J. M. Rawls, General Atomic Co., P.0. Box 81608, San Diego, CA 92138

175. P. J. Reardon, Princeton Plasma Physics Laboratory, Princeton University, P.0. Box 451, Princeton, NJ 08540

176. E. E. Reis, General Atomic Co., P.0. Box 81608, San Diego, CA 92138

177. D. R. Riley, CRBRP Project Office, P.O. Box U, Oak Ridge, TN 37830

178. M. Roberts, Office of Fusion Energy, ETM, Department of Energy, Mail Stop G-234, Washington, DC. 20545 
179. J. D. Rogers, Los Alamos Scientific Laboratory, P.0. Box 1663, Los Alamos, NM 87545

180. S. N. Rosenwasser, General Atomic Co., P.0. Box 81608, 15/219, San Diego, CA 92138

181. P. Rutherford, Princeton Plasma Physics Laboratory, Princeton University, P.0. Box. 451, Princeton, NJ 08540

182. J. Scharer, Department of Nuclear Engineering, University of Wisconsin, Engineering Research Bldg., Madison, WI 53706

183. J. A. Schmidt, Princeton Plasma Physics Laboratory, Princeton University, P.0. Box 451, Princeton, NJ 08540

184. J. Schultz, Massachusetts Institute of Technology, 167 Albany St., Cambridge, MA 02139

185. F. R. Scott, Electric Power Research Institute (EPRI). Fusion Program, 3412 Hillview Ave., P.0. Box 10412, Palo Alto, CA 94304

186. F. Seibel, Princeton Plasma Physics Laboratory, Princeton University, P.0. Box 451, Princeton, NJ 08540

187. Z. M. Shapiro, Manager, Fusion Power Systems, Westinghouse Electric Corp., P.0. Box 10864, Pittsburgh, PA 15236

188. G. Sheffield, Princeton Plasma Physics Laboratory, Princeton University, P.0. Box 451, Princeton, NJ

189. T. J. M. Sluyters, Brookhaven National Laboratory, Accelerator Department, Upton, Long Island, NY 11973

190. D. Smith, Materials Science Division, Argonne National Laboratory, 9700 South Cass Ave., Argonne, IL 60439

191. R. I. Smith, Board Chairman, Public Service Electric and Gas Co., 80 Park Place, Newark, NJ 07101

192. M. Sniderman, Westinghouse Electric Corporation, Fusion Project, 1823 Wightman St.; Pittsburgh, PA 15217

193. L. Southworth, General Atomic Co., P.O. Box 81608, San Diego, CA 92138

194. W. M. Stacey, Jr., Georgia Institute of Technology, School of Nuclear Engineering, Atlanta, GA 30332

195. M. Stauber, Grumman Aerospace Corp., Bethpage, NY 11714

196. L. D. Stewart, Princeton Plasma Physics Laboratory, Princeton University, P.0. Box 451, Princeton, NJ 08540

197. J. L. Straalsund, Westinghouse-Hanford, P.O. Box 1970, Richland, WA 99352

198. I. N. Sviatoslavsky, University of Wisconsi.n, Lb00 Johnsun Drive, Rm. 333 ERB, Madison, WI 53706

199. T. Tamano, General Atomic Co., P.0. Box 81608, San Diego, CA 92138

200. R. E. Tatro, Manager, Energy Systems, General Dynamics, Convair Division, P.O. Box 80847, M.Z. 16-1070, San Diego, CA 92138

201. C. E. Taylor, Lawrence Livermore Laboratory, P.0. Box 808, Livermore, CA 94550

202. F. Tenney, Princeton Plasma Physics. Laboratury, Princeton University, P.0. Box 451, Princeton, NJ 08540

203. K. I. Thomassen, Lawrence Livermore Laboratory, P.0. Box 808, Livermore, CA 94550

204. R. J. Thome, Massachusetts Institute of Technology, Bldg. NW-16-229, 170 Albany St., Cambridge, MA 02139

205. C. Trachse1, McDonne11-Douglas Astronautics Co., P.0. Box 516, St. Louis, Mn 63166 
206. J. R. Treglio, General Dynamics, Convair Division, P.0. Box 80847, San Diego, CA 92138

207. A. W. Trivelpiece, Science Applications, Inc., 1200 Prospect St., P.0. Box 2351, La Jolla, CA 92038

208. T. C. Varljen, Westinghouse Electric Corp., P.0. Box 10864, Pittsburgh, PA 15236

209. A. Wait, Project Engineer, Advanced Applications, Bldg. 36-421, General Electric Co., 1 River Rd., Schenectady, NY 12345

210. K. E. Wakefield, Princeton Plasma Physics Laboratory, Princeton University, P.0. Box 451, Princeton, NJ 08540

211. D. Weldon, Los Alamos Scientific Laboratory, P.0. Box 1663, Mail Stop 464, Los Alamos, NM 87545

212. F. G. Welfare, Babcok and Wilcox, Lynchburg Research Center, P.0. Box 1260, Lynchburg, VA 24505

213. W. R. Wilkes, Monsanto Research Corp., Mound I.aboratory, Miamisburg, OH 45342

214. J. E. Wilkins, EG\&G Idaho, Idaho National Engineering Laboratory, Idaho Falls, ID 83401

215. H. Willenberg, Mathematical Sciences, Northwest, P.0. Bux 1887, Bellevue, WA 98009

216. J. E. C. Williams, Bldg. NW 16-3210, Massachusetts Institute of Technology, 170 Albany St., Cambridge, MA 02139

217. J. M. Williams, Los Alamos Scientific Laboratory, P.0. Box 1663, Los Alamos, NM 87545

218. P. Willis, General Electric Co., 1 River Road, Bldg. 23, Rm. 298, Schenectady, NY 12345

219. K. E. Wright, Princeton Plasma Physics Laboratory, Princeton University, P.0. Box 451, Princeton, NJ 08540

220. T. F. Yang, Massachusetts Institute of Technology, Plasma Fusiun Center, 167 Albany Street, Cambridge, MA 02139

221. H. H. Yoshikawa, Hanford Engineering DcvclopmenL Luboratory W/A-62, P.0. Bóx 1970, Richland, WA 99352

222. S. Yoshikawa, Princeton Plasma Physics Laboratory, Princecon University, P.0. Box 451, Princetun, NJ 08540

223. N. F. Young, EBASCO Services, Inc., Princeton Plasma Physics Laboratory, Princeton University, P.0. Box 451, Princeton, NJ $085 / 10$

224. K. M. Zwilsky, Office of Fusion Energy, ETM, Department of Energy, Mail Stop G-234, Washington, DC 20545

225. Office of Assictant Manager for Energy Kesearch and Development, Department of Energy, Oak Ridge Operations Office, Oak Ridge, TN 37830

226-363. Given distribution as shown in TTD-4500, Magnetic Fusion Energy (Distribution Category UC-20 d, Fusion Systems) 